\title{
Uma Torre de Extensões de Artin-Schreier Atingindo a Cota de Drinfeld-Vladut
}

Herivelto Martins Borges Filho

Dissertação apresentada

ao

Instituto de Matemática e Estatística

da

Universidade de São Paulo

para

obtenção do grau

de

Mestre em Matemática

Área de Concentração: Matemática

Orientador: Prof. Dr. Orlando Stanley Juriaans

São Paulo - Agosto - 2003 


\title{
Uma Torre de Extensões de Artin-Schreier Atingindo \\ a Cota de Drinfeld-Vladut
}

\author{
Este exemplar corresponde à redação \\ final da dissertação devidamente corrigida \\ e defendida por Herivelto Martins Borges \\ Filho e aprovada pela comissão julgadora.
}

São Paulo, 25 de Agosto de 2003.

Banca examinadora:

- Prof. Dr. Orlando Stanley Juriaans (orientador) - IME - USP

- Prof. Dr. Ricardo Bianconni - IME - USP

- Prof. Dr. Nivaldo Nunes de Medeiros Junior - Inst. de Matemática - UFF 


\section{Resumo}

Neste trabalho, apresentamos a construção de uma torre de corpos de funções algébricas: $F_{n} / \mathbb{F}_{q^{2}}$ que atinge a cota de Drinfeld -Vladut . Usando a teoria da ramificação de lugares, calculamos para cada n, o gênero $g_{n}$ de $F_{n}$ bem como o número de lugares de grau $1, N_{n}$. Fazendo o limite, obtemos $\lim _{n \rightarrow \infty} \frac{N_{n}}{g_{n}}=q-1$. 


\section{Abstract}

In this work, we present a construction of a tower of algebraic function fields: $F_{n} / \mathbb{F}_{q^{2}}$ that attains the Drinfeld-Vladut bound.Using the ramification places theory, we calculate for each n, the genus $g_{n}$ of $F_{n}$ as well as the number of places of degree one, $N_{n}$. Evaluating the limit, we obtain $\lim _{n \rightarrow \infty} \frac{N_{n}}{g_{n}}=q-1$. 


\section{Capítulo 1}

\section{Introdução}

O trabalho a seguir baseia-se no artigo intitulado A Tower of Artin-Schreier Extensions of Function Fields Attaining the Drinfeld-Vladut Bound, de Arnaldo Garcia e Henning Stichtenoth, publicado em 1995 (ver [3]).

No intuito de esclarecer a essência do resultado deste trabalho, faremos algumas considerações iniciais. Consideramos $F / \mathbb{F}_{q}$ um corpo de funções algébricas de uma variável, onde $\mathbb{F}_{q}$ (corpo finito com $q$ elementos) é algebricamente fechado em $F$. Se $N=N(F)$ e $g=g(F)$ são, respectivamente, o número de lugares de grau 1 e o gênero de $F / \mathbb{F}_{q}$, então um célebre teorema devido à André Weil ([1], [4] e [13]) afirma que:

$$
N \leq 1+q+2 g \sqrt{q} \quad(1) \text {. }
$$

Em geral, tal cota superior para $N$ não pode ser melhorada, pois existem corpos de funções para os quais ocorre a igualdade em (1). No entanto, quando $q$ não é um quadrado, Serre ([4], [10]) mostra que:

$$
N \leq 1+q+g[2 \sqrt{q}] \quad(2),
$$

onde $[x]$ denota a parte inteira de $x \in R$.

Ihara ([4], [8]) mostra que, se $g$ é suficientemente grande em relação a $q$, então obtemos uma desigualdade estrita em (1), ou, mais precisamente,

$$
g>\frac{q-\sqrt{q}}{2} \Longrightarrow N<1+g+2 g \sqrt{q} \text {. }
$$

Consideremos o número

$$
N_{q}(g)=\max \left\{N(F): F \text { é um corpo de funções algébricas sobre } \mathbb{F}_{q} \text { de gênero } g\right\} \text {. }
$$

Para estudar o comportamento de $N_{q}(g)$, com $g$ arbitrariamente grande, definimos:

$$
A(q):=\limsup _{g \rightarrow \infty} \frac{N_{q}(g)}{g}
$$

Usando (2), temos que:

$$
A(q) \leq[2 \sqrt{q}]
$$




\section{Capítulo 2}

\section{Fundamentos da Teoria de Corpos de Funções Algébricas}

O objetivo principal deste capítulo é apresentar as definições e os resultados básicos da teoria de corpos de funções algébricas. Ressaltamos que todos os resultados, inclusive demonstrações, podem ser encontradas em [11].

\subsection{Lugares}

Definição 2.1. Seja $K$ um corpo. Um corpo de funções algébricas de uma variável $F / K$, sobre $K$, é uma extensão de corpo $F \supseteq K$, onde $F$ é uma extensão finita de $K(x)$ para algum $x \in F$ transcendente sobre $K$.

Por simplicidade chamaremos $F / K$ apenas de corpo de funções. O fecho algébrico de $K$ em $F$, denotado por

$$
\tilde{K}:=\{z \in F \quad: z \text { é algébrico sobre } K\}
$$

será chamado de corpo das constantes de $F$. Como $K \subseteq \tilde{K} \subseteq F$, é fácil verificar que $F / \tilde{K}$ é um corpo de funções sobre $\tilde{K}$.

Observação 2.2. Os elementos de $F$ que são transcendentes sobre $K$, podem ser caracterizados da seguinte forma: $z \in F$ é transcendente sobre $K$ se, e somente se, $[F: K(z)]<$ $\infty$.

Definição 2.3. Um anel de valorização de um corpo de funções $F / K$ é um anel $O \subseteq F$ com as seguintes propriedades:

(1) $K \subsetneq O \subsetneq F, e$

(2) $z \in F \Longrightarrow z \in O$ ou $z^{-1} \in O$.

Proposição 2.4. Seja $O$ um anel de valorização de um corpo de funções $F / K$. Então: 
(a) $O$ é um anel local, isto é, $O$ tem um único ideal maximal $P=O \backslash O^{*}$, onde $O^{*}$ é o grupo das unidades de $O$;

(b) se $0 \neq x \in F$, então $x \in P \Longleftrightarrow x^{-1} \notin O$;

(c) se $\tilde{K}$ é o corpo das constantes de $F / K$, temos $\tilde{K} \subseteq O$ e $P \cap \tilde{K}=\{0\}$.

Teorema 2.5. Seja $O$ um anel de valorização de $F / K$ e $P$ seu ideal maximal. Então:

(a) $P$ é um ideal principal;

(b) se $P=t O$, entấo todo $0 \neq z \in F$ tem uma única representação na forma $z=t^{n} \cdot u$, para algum $n \in \mathbb{Z}$ e algum $u \in O^{*}$;

(c) O é um domínio de ideais principais; mais precisamente, se $P=t O$ e $\{0\} \neq I \subseteq O$ é um ideal, entâo $I=t^{n} O$ para algum $n \in \mathbb{N}$.

Um anel com as propriedades acima é chamado de anel de valorização discreta.

Definição 2.6. (a) Um lugar $P$ de um corpo de funções $F / K$ é o ideal maximal de algum anel de valorização $O$ de $F / K$. O elemento $t \in P$, tal que $P=t O$ é chamado de elemento primo de $P$.

(b) $\mathbb{P}(F):=\{P: P$ é um lugar de $F / K\}$.

Se $O$ é um anel de valorização de $F / K$, e $P$ o seu ideal maximal, então, pelo item (b) da proposição 2.4, $O$ está unicamente determinado por $P$, a saber:

$$
O=\left\{z \in F: z^{-1} \notin P\right\} \cup\{0\} .
$$

Portanto, $O_{P}:=O$ será chamado anel de valorização do lugar $P$.

Definição 2.7. Uma valorização discreta de $F / K$ é uma função sobrejetora $v: F \longrightarrow$ $\mathbb{Z} \cup\{\infty\}$ com as seguintes propriedades:

(1) $v(x)=\infty \Longleftrightarrow x=0$;

(2) $v(x y)=v(x)+v(y), \forall x, y \in F$;

(3) $v(x+y) \geq \min \{v(x), v(y)\}, \forall x, y \in F$;

(4) $v(a)=0, \forall a \in K^{*}$.

A propriedade (3) é chamada de desigualdade triangular. Uma forte conseqüência desta propriedade é apresentada no seguinte lema:

Lema 2.8 (Desigualdade triangular estrita). Seja $v$ uma valorização discreta de $F / K$, $e x, y \in F \operatorname{com} v(x) \neq v(y)$. Então $v(x+y)=\min \{v(x), v(y)\}$. 
Definição 2.9. A cada lugar $P \in \mathbb{P}(F)$ associamos a função $v_{P}: F \longrightarrow \mathbb{Z} \cup\{\infty\}$, da seguinte forma: se $0 \neq z \in F$, então $z$ é unicamente representado na forma $z=t^{n} \cdot u$, com $u \in O^{*}$ e $n \in \mathbb{Z} ;$ assim, definimos $v_{P}(z):=n$ e $v_{P}(0):=\infty$.

Teorema 2.10. Seja $F / K$ um corpo de funçôes.

(a) Para todo lugar $P \in \mathbb{P}(F)$, a função vP definida acima é uma valorização discreta, $e$ ainda temos que:

$$
\begin{gathered}
O_{P}=\left\{z \in F \quad: \quad v_{P}(z) \geq 0\right\}, \\
O_{P}^{*}=\left\{z \in F \quad: \quad v_{P}(z)=0\right\}, \\
P=\left\{z \in F \quad: \quad v_{P}(z)>0\right\},
\end{gathered}
$$$$
x \in F \text { é elemento primo de } P \Longleftrightarrow v_{P}(x)=1 \text {. }
$$

(b) Reciprocamente, suponhamos que $v$ seja uma valorização discreta de $F / K$. Entấo o conjunto $P:=\{z \in F: v(z)>0\}$ é um lugar de $F / K$, e $O_{P}=\{z \in F: v(z) \geq$ $0\}$ é o anel de valorização correspondente.

(c) Todo anel de valorização $O$ de $F / K$ é um subanel maximal de $F$.

Entre outras coisas, o teorema acima nos mostra que os lugares e anéis de valorização de um corpo de funções $F / K$ podem ser descritos por uma valorização $v: F \longrightarrow \mathbb{Z} \cup\{\infty\}$, ou seja, tais noções são equivalentes.

Seja $P$ um lugar de $F / K$ e $O_{P}$ seu anel de valorização. Como $P$ é ideal maximal de $O_{P}$, temos que o quociente $O_{P} / P$ é um corpo. Para $x \in O_{P}$, definimos $x(P) \in O_{P} / P$ como sendo a classe de resíduos de $x$ módulo $P$. Para $x \in F \backslash O_{P}$, definimos $x(P):=\infty$. Assim, pelo item $c$ da proposição 2.4 , temos, de forma canônica, a imersão $K \hookrightarrow O_{P} / P$. Portanto, sempre consideraremos $K$ como subcorpo de $O_{P} / P$. Os mesmos argumentos se aplicam a $\tilde{K}$.

Definição 2.11. Seja $P \in \mathbb{P}(F)$.

(a) $\mathbb{F}_{P}:=O_{P} / P$ é chamado de corpo residual de $P$. A função $x \mapsto x(P)$ de $F$ para $\mathbb{F}_{P} \cup\{\infty\}$ é chamada de função de classe de resíduo com respeito a $P$. Em alguns momentos usaremos a notação $x+P:=x(P)$ para $x \in O_{P}$.

(b) $\operatorname{deg}(P):=\left[\mathbb{F}_{P}: K\right]$ é chamado de grau de $P$.

Proposição 2.12. Se $P$ é um lugar de $F / K$ e $0 \neq x \in P$, então $\operatorname{deg}(P) \leq[F: K(x)]$. Portanto, $\mathbb{F}_{P}$ é uma extensão finita de $K$.

Corolário 2.13. Supondo $\mathbb{P}(F) \neq \varnothing$, temos que o corpo $\tilde{K}$ das constantes de $F / K$ é uma extensão finita de $K$. 
Demonstração. Imediato, pois $K \subseteq \tilde{K} \subseteq \mathbb{F}_{P}$.

Observação 2.14. Se deg $(P)=1$, então $\mathbb{F}_{P}=K$, e a função da classe de resíduos leva $F$ em $K \cup\{\infty\}$. Em particular, se $K$ é algebricamente fechado, então todo lugar de $F / K$ tem grau 1 .

Definição 2.15. Seja $z \in F$ e $P \in \mathbb{P}(F)$. Dizemos que $P$ é zero de $z$ se, e somente se, $v_{P}(z)>0 ; P$ é pólo se, e somente se, $v_{P}(z)<0$. $P$ é zero de ordem $m$, se $v_{P}(z)=m>0$, e P é pólo de ordem $m$ se $v_{P}(z)=m<0$.

Apesar de toda teoria descrita até aqui, nada assegurou a existência de anel de valorização, e, portanto, de lugares. O próximo teorema nos garante tal fato.

Teorema 2.16. Seja $F / K$ um corpo de funções e $R$ um subanel de $F$ com $K \subseteq R \subseteq F$. Suponha que $\{0\} \neq I \subsetneq R$ seja um ideal próprio de $R$. Então existe um lugar $P \in \mathbb{P}(F)$ tal que $I \subseteq P$ e $R \subseteq O_{P}$.

Observe que tal subanel $R$ (que não é corpo) sempre existe, pois basta tomar $z \in F$ transcendente sobre $K$ e fazer $R=K[z]$.

Corolário 2.17. Seja $F / K$ um corpo de funções, $z \in F$ transcendente sobre $K$. Então $z$ tem pelo menos um zero e um pólo. Em particular, $\mathbb{P}(F) \neq \varnothing$.

Demonstração. Considere o anel $R=K[z]$ e o ideal $I=z K[z]$. Pelo teorema anterior, existe um lugar $P \in \mathbb{P}(F)$ tal que $z K[z] \subseteq P \Longrightarrow z \in P$, e, portanto, $P$ é zero de $z$. O mesmo argumento mostra que $z^{-1}$ tem um zero $Q \in \mathbb{P}(F)$. Logo, $Q$ é pólo de $z$.

\subsection{Corpo de Funções Racionais}

Apresentaremos agora o exemplo mais simples de corpo de funções algébricas.

Definição 2.18. Um corpo de funções $F / K$ é chamado de corpo de funções racionais se $F=K(x)$ para algum $x \in F$ transcendente sobre $K$.

Dado um polinômio qualquer $p(x) \in K[x]$, irredutível, é fácil ver que o conjunto

$$
O_{p(x)}:=\left\{\frac{f(x)}{g(x)}: f(x), g(x) \in K[x] \text { e } p(x) \nmid g(x)\right\}
$$

é um anel de valorização de $K(x) / K$, e que

$$
P_{p(x)}:=\left\{\frac{f(x)}{g(x)}: f(x), g(x) \in K[x], \text { onde } p(x) \nmid g(x) \text { e } p(x) \mid f(x)\right\}
$$

é o lugar associado a $O_{p(x)}$. No caso particular em que $p(x)$ é linear, isto é, $p(x)=x-\alpha$ com $\alpha \in K$, escreveremos abreviadamente $P_{\alpha}:=P_{x-\alpha} \in \mathbb{P}_{K(x)}$. 
Existe um outro tipo de anel de valorização de $K(x) / K$, dado por

$$
O_{\infty}=\left\{\frac{f(x)}{g(x)} \quad: \quad f(x), g(x) \in K[x] \text { e } \operatorname{deg}(g(x)) \geq \operatorname{deg}(f(x))\right\}
$$

cujo ideal maximal associado é:

$$
\text { (4) } \quad P_{\infty}=\left\{\frac{f(x)}{g(x)}: f(x), g(x) \in K[x] \text { e } \operatorname{deg}(g(x))>\operatorname{deg}(f(x))\right\}
$$

Este último é denominado lugar infinito de $K(x)$.

Proposição 2.19. Seja $F=K(x)$ um corpo de funções racionais.

(a) Seja $P_{\boldsymbol{p}(x)} \in \mathbb{P}_{K(x)}$, onde $p(x) \in K[x]$ é um polinômio irredutível. Então, $p(x)$ é um elemento primo de $P$, e a valorização discreta pode ser descrita da seguinte forma: se $z \in K(x) \backslash\{0\}$ é escrito da forma

$$
z=p(x)^{n} \cdot\left(\frac{f(x)}{g(x)}\right), \text { com } n \in \mathbb{Z}, f(x), g(x) \in K[x], p(x) \nmid f(x) \text { e } p(x) \nmid g(x),
$$

então $v_{P}(z)=n$. O corpo residual $K(x)_{P}=O_{P} / P$ é isomorfo a $K[x] /\langle p(x)\rangle$, e tal isomorfismo é dado por:

$$
\phi:\left\{\begin{array}{cl}
K[x] /\langle p(x)\rangle & \longrightarrow K(x)_{P} \\
f(x) \bmod p(x) & \longmapsto f(x)(P)
\end{array} .\right.
$$

Conseqüentemente, $\operatorname{deg}(P)=\operatorname{deg}(p(x))$.

(b) No caso especial em que $p(x)=x-\alpha$, com $\alpha \in K$, temos $\operatorname{deg}(P)=1$.

(c) Considere $P_{\infty} \in \mathbb{P}_{K(x)}$ definido em (4). Então, $\operatorname{deg}\left(P_{\infty}\right)=1$. Um elemento primo de $P_{\infty}$ é $t=\frac{1}{x}$. A valorização discreta $v_{\infty}$ é dada por:

$$
v_{\infty}\left(\frac{f(x)}{g(x)}\right)=\operatorname{deg}(g(x))-\operatorname{deg}(f(x))
$$

(d) $K=\tilde{K}$, isto é, $K$ é o corpo das constantes de $K(x) / K$.

Teorema 2.20. Os únicos lugares de $K(x) / K$ são os lugares do tipo $P_{p(x)}$ e $P_{\infty}$, definidos $e m(2) e(4)$.

Corolário 2.21. Os lugares de $K(x) / K$ de grau 1 , estão em correspondência biunívoca com $K \cup\{\infty\}$.

Demonstração. Decorre imediatamente do item (a) da proposição 2.19 e do teorema acima. O diagrama abaixo retrata esta bijeção:

$\{$ lugares de grau 1 de $K(x) / K\} \longrightarrow \alpha \cup\{\infty\}$ 


\subsection{Independência de Valorizações}

Teorema 2.22 (Aproximação fraca). Seja $F / K$ um corpo de funções e $P_{1}, P_{2}, \ldots, P_{n} \in$ $\mathbb{P}(F)$ lugares dois a dois distintos de $F / K$. Se $x_{1}, x_{2}, \ldots, x_{n} \in F$ e $r_{1}, r_{2}, \ldots, r_{n} \in \mathbb{Z}$, então existe $0 \neq x \in F$ tal que

$$
v_{P_{i}}\left(x-x_{i}\right)=r_{i} \text { para } i=1,2, \ldots, n \text {. }
$$

Corolário 2.23. Todo corpo de funções $F / K$ tem infinitos lugares.

Demonstração. Suponhamos que $\# P(F)=: n<\infty$, e tomemos $x_{i}=0$ e $r_{i}>0$ para todo $i=1,2, \ldots, n$. Pelo teorema acima, existe $x \in F$ tal que $v_{P_{i}}(x)>0$ para todo $i=1,2, \ldots, n$, ou seja, $x$ é transcendente e não tem pólos, contrariando o corolário 2.17 .

Proposição 2.24. Seja $F / K$ um corpo de funções e $P_{1}, \ldots, P_{r}$ zeros de um elemento $x \in F$. Então,

$$
\sum_{i=1}^{r} v_{P_{i}}(x) \cdot \operatorname{deg}\left(P_{i}\right) \leq[F: K(x)]
$$

Corolário 2.25. Em um corpo de funções $F / K$, todo elemento $0 \neq x \in F$ tem um número finito de zeros e pólos.

Demonstração. Se $x$ é uma constante, então $x$ não tem zeros nem pólos. Se $x$ é transcendente e tem infinitos zeros, então é possível tomar um inteiro positivo $r$ tal que

$$
\sum_{i=1}^{r} v_{P_{i}}(x) \cdot \operatorname{deg}\left(P_{i}\right)>[F: K(x)],
$$

contrariando a proposição acima. Logo, $x$ tem um número finito de zeros. Usando o mesmo argumento com $x^{-1}$, concluímos que $x$ tem um número finito de pólos.

\subsection{Divisores}

Como o corpo das constantes $\tilde{K}$ de $F / K$ é uma extensão finita de $K$, e $F / \tilde{K}$ é um corpo de funções, consideraremos, de agora em diante, que $K$ seja o corpo das constantes de $F / K$, ou seja, $K$ algebricamente fechado em $F$.

Definição 2.26. O grupo (aditivo) abeliano livre gerado pelos lugares de $F / K$ será chamado de grupo dos divisores de $F / K$, e denotado por $D_{F}$. 
Os elementos de $D_{F}$ serão chamados de divisores de $F / K$. Formalmente, um divisor $D$ é dado por

$$
D=\sum_{P \in \mathbb{P}(F)} n_{P} P
$$

com $n_{P} \in \mathbb{Z}$ e $n_{P}=0$ para quase todo $P \in \mathbb{P}(F)$. Algumas definições e fatos serão apresentados a seguir.

- Um divisor da forma $D=P$, com $P \in \mathbb{P}(F)$, é chamado divisor primo.

- Se $D=\sum n_{P} P$ e $D^{\prime}=\sum n_{P}^{\prime} P$ então $D+D^{\prime}=\sum\left(n_{P}+n_{P}^{\prime}\right) P$.

- O elemento nulo do grupo $D_{F}$ é o divisor

$$
0:=\sum_{P \in \mathbb{P}(F)} r_{P} P
$$

onde $r_{P}=0$ para todo $P \in \mathbb{P}(F)$.

- Se $Q \in \mathbb{P}(F)$ e $D=\sum n_{P} P \in D_{F}$, definimos

$$
v_{Q}(D):=n_{Q} \quad \text { e } \operatorname{supp} D:=\left\{P \in \mathbb{P}(F): v_{P}(D) \neq 0\right\}
$$

sendo este último chamado de suporte de $D$.

- Definimos uma ordem parcial em $D_{F}$ da seguinte forma:

$$
D_{1} \leq D_{2} \Longleftrightarrow v_{P}\left(D_{1}\right) \leq v_{P}\left(D_{2}\right), \forall P \in \mathbb{P}(F) .
$$

Um divisor $D \geq 0$ é denominado positivo.

- O grau de um divisor é definido por

$$
\operatorname{deg}(D)=\sum_{P \in \mathbb{P}(F)} v_{P}(D) \cdot \operatorname{deg}(P)
$$

originando um homomorfismo deg: $D_{F} \longrightarrow \mathbb{Z}$.

Pelo corolário 2.25, todo elemento $0 \neq x \in F$ tem um número finito de zeros e pólos em $\mathbb{P}(F)$. Este fato dá consistência à próxima definição.

Definição 2.27. Seja $0 \neq x \in F$, e $Z$ e $N$ os conjuntos de zeros e pólos de $x$ em $\mathbb{P}(F)$, respectivamente. Então, definimos:

$$
\begin{aligned}
(x)_{0} & :=\sum_{P \in Z} v_{P}(x) P, \text { o divisor de zeros de } x \\
(x)_{\infty} & :=\sum_{P \in N}\left(-v_{P}(x)\right) P, \text { o divisor de pólos de } x \\
(x) & :=(x)_{0}-(x)_{\infty}, \text { o divisor principal de } x
\end{aligned}
$$


Obviamente, temos:

$$
(x)_{0} \geq 0 \quad, \quad(x)_{\infty} \geq 0 \quad \text { e } \quad(x)=\sum_{P \in \mathbb{P}(F)} v_{P}(x) P
$$

Os elementos $0 \neq x \in F$ que são constantes são evidentemente caracterizados por

$$
x \in K \Longleftrightarrow(x)=0
$$

O próximo teorema, grosso modo, nos diz que um elemento $0 \neq x \in F$ tem o mesmo número de zeros e pólos se levarmos em conta as multiplicidades.

Teorema 2.28. Todo divisor principal tem grau zero. Mais precisamente: seja $x \in F / K$ $e(x)_{0},(x)_{\infty}$ os divisores zero e pólo de $x$, respectivamente. Então:

$$
\operatorname{deg}(x)_{0}=\operatorname{deg}(x)_{\infty}=[F: K(x)]
$$

Definição 2.29. (a) $\mathcal{P}_{F}:=\{(x): 0 \neq x \in F\}$ é chamado de grupo dos divisores principais de $F / K . \mathcal{P}_{F}$ é um subgrupo de $D_{F}$, pois se $0 \neq x, y \in F$, temos $(x y)=$ $(x)+(y)\left(\right.$ pois, $\left.v_{P}(x y)=v_{P}(x)+v_{P}(y)\right)$.

(b) $O$ grupo quociente $\mathcal{C}_{F}:=D_{F} / \mathcal{P}_{F}$ é chamado de grupo de classes de divisor.

(c) Para um divisor $D \in D_{F}$, o elemento correspondente em $\mathcal{C}_{F}$ será denotado por $[D]$, e chamado a classe do divisor $D$. Dois divisores $D, D^{\prime} \in D_{F}$ são considerados equivalentes (e escrevemos $D \sim D^{\prime}$ ) se $[D]=\left[D^{\prime}\right]$, isto é,

$$
D-D^{\prime} \in \mathcal{P}_{F} \Longrightarrow D=D^{\prime}+(x)
$$

para algum $x \in F \backslash\{0\}$.

Definição 2.30. Para um divisor $A \in D_{F}$, temos:

$$
\mathcal{L}(A):=\{x \in F \quad:(x) \geq-A\} \cup\{0\} .
$$

Esta definição tem a seguinte interpretação: se

$$
A=\sum_{i=1}^{r} n_{i} P_{i}-\sum_{j=1}^{s} m_{j} Q_{j}
$$

com $n_{i}>0$ e $m_{j}>0$, então $\mathcal{L}(A)$ consiste de todos os elementos $x \in F$ tais que

(1) $Q_{j}$ é zero de $x$ com ordem maior ou igual a $m_{j}(j=1, \ldots, s)$;

(2) $P_{1}, \ldots, P_{r}$ são os únicos possíveis pólos de $x$, cuja ordem (de pólo) é menor ou igual a $n_{i}(i=1, \ldots, r)$. 
Lema 2.31. Seja $A \in D_{F}$. Então, temos:

(a) $\mathcal{L}(A)$ é um espaço vetorial sobre $K$;

(b) se $A^{\prime} \sim A$, então $\mathcal{L}(A) \cong \mathcal{L}\left(A^{\prime}\right)$ (são isomorfos como espaços vetoriais), e $\operatorname{deg}(A)=$ $\operatorname{deg}\left(A^{\prime}\right)$

(c) se $A$ é o divisor nulo, então $\mathcal{L}(A)=K$;

(d) se $A<0$, então $\mathcal{L}(A)=\{0\}$;

(e) se $B \in D_{F}$ e $B \geq A$, então $\mathcal{L}(A) \subseteq \mathcal{L}(B)$ e $\operatorname{dim}(\mathcal{L}(B) / \mathcal{L}(A)) \leq \operatorname{deg}(B)-\operatorname{deg}(A)$.

Proposição 2.32. Se $A \in D_{F}$, então $\mathcal{L}(A)$ é um $K$-espaço vetorial de dimensão finita. Mais precisamente, se $A=A_{+}-A_{-}$, onde $A_{+}$e $A_{-}$são divisores positivos, então:

$$
\operatorname{dim}(\mathcal{L}(A)) \leq \operatorname{deg}\left(A_{+}\right)+1 .
$$

Demonstração. (Os itens a que nos reportaremos serão os do lema anterior.)

Como $A \leq A_{+}$, pelo item $(e)$, temos $\mathcal{L}(A) \subseteq \mathcal{L}\left(A_{+}\right)$, e, assim, é suficiente mostrar que $\operatorname{dim}\left(\mathcal{L}\left(A_{+}\right)\right) \leq \operatorname{deg}\left(A_{+}\right)+1$.

Mas, também temos $0 \leq A_{+}$, e, portanto, novamente por $(e)$, segue que

$$
\operatorname{dim}\left(\mathcal{L}\left(A_{+}\right) / \mathcal{L}(0)\right) \leq \operatorname{deg}\left(A_{+}\right) .
$$

Agora, pelo item $(c)$, temos $\mathcal{L}(0)=K$. Logo:

$$
\operatorname{dim}\left(\mathcal{L}\left(A_{+}\right)\right)-1 \leq \operatorname{deg}\left(A_{+}\right) \Longrightarrow \operatorname{dim}\left(\mathcal{L}\left(A_{+}\right)\right) \leq \operatorname{deg}\left(A_{+}\right)+1 .
$$

Definição 2.33. Se $A \in D_{F}$, o número inteiro $\operatorname{dim}(A):=\operatorname{dim}(\mathcal{L}(A))$ é chamado a dimensão do divisor $A$.

Teorema 2.34. Seja $F / K$ um corpo de funções. Então existe $\gamma \in \mathbb{Z}$, dependendo de $F / K$, tal que, para todo divisor $A \in D_{F}$, temos $\operatorname{deg}(A)-\operatorname{dim}(A) \leq \gamma$.

A seguir, definiremos um dos mais importantes invariantes de um corpo de funções.

Definição 2.35. O gênero de $F / K$ é definido por

$$
g:=\max \left\{\operatorname{deg}(A)-\operatorname{dim}(A)+1: A \in D_{F}\right\} .
$$

Observamos, pelo teorema anterior, que tal definição é consistente.

Observação 2.36. O gênero de $F / K$ é um inteiro não-negativo. De fato, tomando o divisor $A=0$, temos:

$$
g \geq \operatorname{deg}(0)-\operatorname{dim}(0)+1=0-1+1=0
$$


Teorema 2.37 (Teorema de Riemann). Seja $F / K$ um corpo de funções de gênero $g$.

(a) Se $A \in D_{F}$, então $\operatorname{dim}(A) \geq \operatorname{deg}(A)+1-g$.

(b) Existe um inteiro $c$, dependendo de $F / K$, tal que $\operatorname{dim}(A)=\operatorname{deg}(A)+1-g$ para todo $A \in D_{F}, \operatorname{com} \operatorname{deg}(A) \geq c$.

Demonstração. (a) Pela definição de gênero, temos:

$$
g \geq \operatorname{deg}(A)-\operatorname{dim}(A)+1 \Longrightarrow \operatorname{dim}(A) \geq \operatorname{deg}(A)+1-g .
$$

(b) Tomemos $A_{0} \in D_{F}$ tal que $g=\operatorname{deg}\left(A_{0}\right)-\operatorname{dim}\left(A_{0}\right)+1$, e seja $c:=\operatorname{deg}\left(A_{0}\right)+g$. Se $\operatorname{deg}(A) \geq c$, temos por $(\mathrm{a})$ :

$$
\begin{aligned}
\operatorname{dim}\left(A-A_{0}\right) & \geq \operatorname{deg}\left(A-A_{0}\right)+1-g \\
& =\operatorname{deg}(A)-\operatorname{deg}\left(A_{0}\right)+1-g \\
& \geq c-\operatorname{deg}\left(A_{0}\right)+1-g \\
& =g+1-g=1 .
\end{aligned}
$$

Logo, $\operatorname{dim}\left(A-A_{0}\right) \geq 1$, e, portanto, existe $0 \neq z \in \mathcal{L}\left(A-A_{0}\right)$, e assim $(z) \geq A_{0}-A$. Considere o divisor $\dot{A}^{\prime}:=A+(z) \geq A_{0}$. Temos:

$$
\begin{aligned}
\operatorname{deg}(A)-\operatorname{dim}(A) & =\operatorname{deg}\left(A^{\prime}\right)-\operatorname{dim}\left(A^{\prime}\right) \quad\left(\text { pois } A \sim A^{\prime}\right) \\
& \geq \operatorname{deg}\left(A_{0}\right)-\operatorname{dim}\left(A_{0}\right) \quad(\text { item (e) do lema 2.31) } \\
& =g-1 .
\end{aligned}
$$

Logo, $\operatorname{dim}(A) \leq \operatorname{deg}(A)+1-g, \mathrm{e}$, portanto, $\operatorname{dim}(A)=\operatorname{deg}(A)+1-g$.

Corolário 2.38. $O$ corpo de funções racionais $K(x) / K$ tem gênero $g=0$.

Demonstração. Seja $P_{\infty}$ o divisor de pólos de $x$. Para $r \geq 0$, considere o espaço vetorial $\mathcal{L}\left(r P_{\infty}\right)$. Observamos que $1, x, \ldots, x^{r} \in \mathcal{L}\left(r P_{\infty}\right)$, pois $v_{P}\left(x^{i}\right) \geq-v_{P}\left(r P_{\infty}\right)$, para todo $P \in \mathbb{P}(F)$ e todo $i=1, \ldots, r$. Sendo $x$ transcendente sobre $K$, temos ainda que:

$$
r+1 \leq \operatorname{dim}\left(r P_{\infty}\right)=\operatorname{deg}\left(r P_{\infty}\right)+1-g=r+1-g
$$

para $r$ suficientemente grande. Logo $g \leq 0$, e, como sabemos que $g \geq 0$ para todo corpo de funções $F / K$, concluímos que $g=0$.

\subsection{O Teorema de Riemann-Roch e Algumas Conseqüências}

Nesta seção, vamos introduzir o conceito de diferencial de Weil, que é fundamental para a apresentação do teorema de Riemann-Roch; no entanto, algumas definições preliminares são necessárias. Doravante, $F / K$ denotará um corpo de funções algébricas de gênero $g$. 
Definição 2.39. Para $A \in D_{F}, i(A):=\operatorname{dim}(A)-\operatorname{deg}(A)+g-1$ é denominado índice de especialidade de $A$.

O teorema de Riemann nos diz que $i(A) \geq 0$, e, se $\operatorname{deg}(A)$ é suficientemente grande, então $i(A)=0$.

Definição 2.40. Um adele de $F / K$ é uma função

$$
\alpha:\left\{\begin{array}{l}
\mathbb{P}(F) \longrightarrow \alpha_{P} \\
P \longmapsto \alpha_{P}
\end{array},\right.
$$

onde $\alpha_{P} \in O_{P}$ para quase todo $P \in \mathbb{P}(F)$. O conjunto $\mathcal{A}_{F}:=\{\alpha: \alpha$ é um adele de $F / K\}$ é chamado espaço dos adeles de $F / K$, pois este pode, de maneira natural, ser considerado como um $K$-espaço vetorial.

Um adele principal de um elemento $x \in F$ é aquela cujas componentes são todas iguais a $x$. Observamos que tal definição é consistente, uma vez que $x \in F$ tem um número finito de pólos em $\mathbb{P}(F)$. Tal definição nos traz automaticamente a imersão $F \hookrightarrow \mathcal{A}_{F}$. A valorização $v_{P}$ de $F / K$ pode ser estendida a $\mathcal{A}_{F}$ fazendo $v_{P}(\alpha)=v_{P}\left(\alpha_{P}\right)$ (onde $\alpha_{P}$ é a $P$-ésima componente do adele $\alpha$ ). Assim, por definição, $v_{P}(\alpha) \geq 0$ para quase todo $P \in \mathbb{P}(F)$.

Definição 2.41. Se $A \in D_{F}$, definimos $\mathcal{A}_{F}(A):=\left\{\alpha \in \mathcal{A}_{F}: v_{P}(\alpha) \geq-v_{P}(A), \forall P \in\right.$ $\mathbb{P}(F)\}$, que é, naturalmente, um $K$-subespaço vetorial de $\mathcal{A}_{F}$.

Definição 2.42. Uma diferencial de Weil de $F / K$ é uma função $K$-linear $\omega: \mathcal{A}_{F} \longrightarrow$ $K$ que se anula em $\mathcal{A}_{F}(A)+F$ para algum $A \in D_{F}$. Denominamos o conjunto

$$
\Omega_{F}:=\{\omega: \omega \text { é uma diferencial de Weil de } F / K\}
$$

de módulo das diferenciais de Weil de $F / K . \Omega_{F}$ pode ser visto como espaço vetorial sobre $K$ de forma natural.

Gostaríamos de associar a cada $0 \neq \omega \in \Omega_{F}$, um divisor $W \in D_{F}$. Para isto, consideremos o conjunto

$$
M(\omega):=\left\{A \in D_{F}: \omega \text { se anula em } \mathcal{A}_{F}(A)+F\right\} .
$$

É possível provar que existe um único divisor $W \in M(\omega)$ tal que

$$
W=\max \left\{A \in D_{F} \quad: \omega \text { se anula em } \mathcal{A}_{F}(A)+F\right\}
$$

Para $0 \neq \omega \in \Omega_{F^{*}}$ e $W \in D_{F}$, obtido da forma acima, usaremos a seguinte notação: $(\omega):=W$. Diremos que um divisor $W$ é canônico se $W=(\omega)$ para algum $\omega \in \Omega_{F}$. 
Lema 2.43. Seja $A$ um divisor arbitrário, e $W=(\omega)$ um divisor canônico de $F / K$. Entâa, $i(A)=\operatorname{dim}(W-A)$.

Teorema 2.44 (Teorema de Riemann-Roch). Seja $W$ um divisor canônico de $F / K$. Então, para $A \in D_{F}$, temos

$$
\operatorname{dim}(A)=\operatorname{deg}(A)+1-g+\operatorname{dim}(W-A) .
$$

Demonstração. Por definição, temos $i(A)=\operatorname{dim}(A)-\operatorname{deg}(A)+g-1$, e, pelo lema anterior, temos $i(A)=\operatorname{dim}(W-A)$. Logo:

$\operatorname{dim}(W-A)=\operatorname{dim}(A)-\operatorname{deg}(A)+g-1 \Longrightarrow \operatorname{dim}(A)=\operatorname{deg}(A)+1-g-\operatorname{dim}(W-A)$.

Corolário 2.45. Para um divisor canônico $W$, temos $\operatorname{deg}(W)=2 g-2$ e $\operatorname{dim}(W)=g$.

Demonstração. Pelo teorema de Riemann-Roch, se fizermos $A=0$, temos:

$\operatorname{dim}(0)=\operatorname{deg}(0)+1-g+\operatorname{dim}(W+0) \Longrightarrow 1=0+1-g+\operatorname{dim}(W) \Longrightarrow \operatorname{dim}(W)=g$,

e, se tomarmos $A=W$, temos

$\operatorname{dim}(W)=\operatorname{deg}(W)+1-g+\operatorname{dim}(0) \Longrightarrow g=\operatorname{deg}(W)+1-g+1 \Longrightarrow \operatorname{dim}(W)=2 g-2$.

Os dois próximos resultados também são conseqüências do teorema de Riemann-Roch, e de grande utilidade no desenvolvimento da teoria de corpos de funções.

Teorema 2.46 (Teorema da aproximação forte). Seja $S \subsetneq \mathbb{P}(F)$ um subconjunto próprio de $\mathbb{P}(F)$, e $P_{1}, \ldots, P_{r} \in S$. Suponha que sejam dados $x_{1}, \ldots, x_{r} \in F$ e $n_{1}, \ldots, n_{r} \in$ $\mathbb{Z}$. Então, existe $x \in F$ tal que

$$
v_{P_{i}}\left(x-x_{i}\right)=n_{i} \text { para } i=1, \ldots, r
$$

$e$

$$
v_{P}(x) \geq 0, \forall P \in S \backslash\left\{P_{1}, \ldots, P_{r}\right\}
$$

Proposição 2.47. Seja $P \in \mathbb{P}(F)$. Então, para $n \geq 2 g$, existe um elemento $x \in F$ cujo divisor de pólos é $(x)_{\infty}=n P$.

Uma das aplicações da proposição acima é poder obter, a partir de um dado lugar $P \in \mathbb{P}(F), x \in F$ que tenha $P$ como seu único zero ou pólo. 


\section{Capítulo 3}

\section{Extensões Algébricas de Corpos de Funções}

Neste Capítulo , a menos de menção em contrário, consideraremos:

- o corpo de funções algébricas $F / K$, com $K$ algebricamente fechado em $F$, isto é, $K=\tilde{K}$

- $K$ um corpo perfeito;

- $\Phi$ um corpo fixo, algebricamente fechado, onde $F \subseteq \Phi$ e todas as extensões $F^{\prime} \supseteq F$ são tais que $F^{\prime} \subseteq \Phi$.

Ressaltamos novamente que as demonstrações apresentadas, bem como aquelas que forem omitidas, podem ser encontradas em [11].

\subsection{Extensões Algébricas de Corpos de Funções}

O principal objetivo desta seção é a introdução de conceitos básicos como:

- extensão algébrica de corpos de funções;

- extensão de lugares;

- índice de ramificação;

- a igualdade fundamental $\sum e_{i} f_{i}=n$.

Tais conceitos serão amplamente utilizados ao longo deste texto. Os dois últimos resultados desta seção (corolário 3.13 e proposição 3.14) também merecem destaque por serem ferramentas importantes para o desenvolvimento do Capítulo 4.

Definição 3.1. (a) Uma extensão algébrica de um corpo de funções $F / K$ é um corpo de funções $F^{\prime} / K^{\prime}$, onde $F^{\prime} \supseteq F$ é uma extensão algébrica e $K \subseteq K^{\prime}$.

(b) Uma extensão algébrica $F^{\prime} / K^{\prime}$ de $F / K$ é dita constante se $F^{\prime}=K^{\prime} F$, ou seja, se $F^{\prime}$ é o compósito de $K^{\prime}$ e $F$. 
(c) Uma extensão algébrica $F^{\prime} / K^{\prime}$ de $F / K$ é dita finita se $\left[F^{\prime}: F\right]<\infty$.

Lema 3.2. Seja $F^{\prime} / K^{\prime}$ uma extensão algébrica de $F / K$. Então:

(a) $K^{\prime} / K$ é uma extensão algébrica, e $F \cap K^{\prime}=K$;

(b) $F^{\prime} / K^{\prime}$ é uma extensão finita de $F / K$ se, e somente se, $\left[K^{\prime}: K\right]<\infty$;

(c) se $F_{1}:=F K^{\prime}$, temos que $F_{1} / K^{\prime}$ é uma extensão constante de $F / K$ e $F^{\prime} / K^{\prime}$ é uma extensão finita de $F_{1} / K^{\prime}$.

Demonstração. (a) $\mathrm{O}$ diagrama abaixo mostra que a extensão $K^{\prime} / K$ é algébrica:

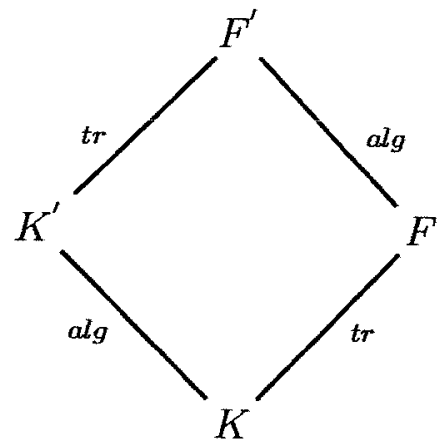

Agora, é claro que $K \subseteq K^{\prime} \cap F^{\prime}$. Seja $z \in K^{\prime} \cap F$; mostraremos que $z \in K$ (consideremos $K$ algebricamente fechado em $F$ ). Suponhamos $z$ transcendente; então, a extensão $K(z) / K$ é transcendente, o que contraria o diagrama abaixo.

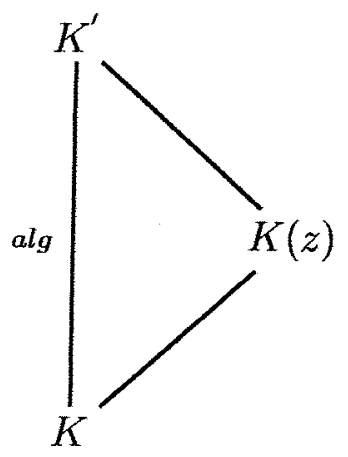

(b) Suponha $\left[F^{\prime}: F\right]<\infty$; então, $F^{\prime} / K$ é um corpo de funções algébricas, e $K^{\prime}$ fará o papel do $\tilde{K}$ definido no Capítulo 2 . Logo, $\left[K^{\prime}: K\right]<\infty$. 


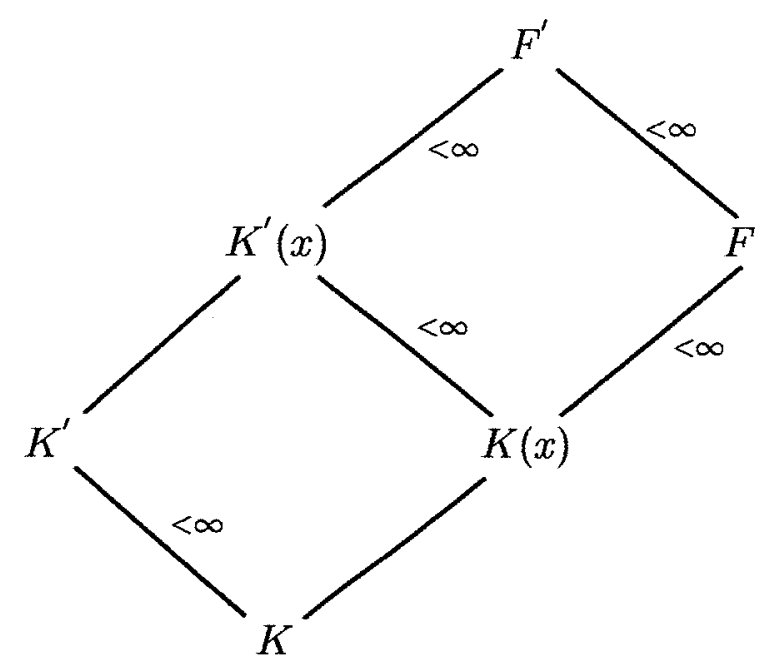

Agora, para mostrarmos a recíproca, assumiremos o seguinte fato: se $K^{\prime} / K$ é uma extensão de corpos finita, e $x$ é transcendente sobre $K$, então $\left[K^{\prime}(x): K(x)\right]=\left[K^{\prime}\right.$ : $K]$ (que é um caso particular do lema 3.46, apresentado mais adiante). Assumindo $\left[K^{\prime}: K\right]<\infty$, e tomando $x \in F$ transcendente sobre $K$, o diagrama acima mostra que $\left[F^{\prime}: F\right]<\infty$.

(c) Usando o item (b), o diagrama abaixo mostra que $\left[F^{\prime}: F_{1}\right]<\infty$.

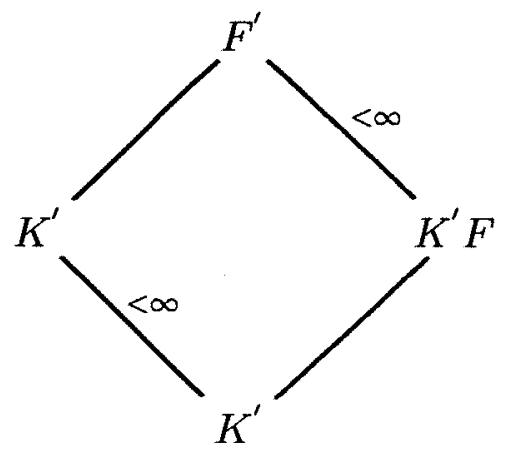

Veremos agora a relação entre os lugares de $F$ e $F^{\prime}$.

Definição 3.3. Considere uma extensão algébrica $F^{\prime} / K^{\prime}$ de $F / K$. Um lugar $P^{\prime} \in \mathbb{P}\left(F^{\prime}\right)$ está sobre $P \in \mathbb{P}(F)$ se $P \subseteq P^{\prime}$. Também dizemos que $P^{\prime}$ é uma extensão de $P$, $e$ denotamos isto por $P^{\prime} \mid P$.

Proposição 3.4. Considere uma extensão algébrica $F^{\prime} / K^{\prime}$ de $F / K$. Tomemos $P, O_{P}$, $v_{P}$ em $F / K$, e $P^{\prime}, O_{P^{\prime}}, v_{P^{\prime}}$ em $F^{\prime} / K^{\prime}$. São equivalentes:

(1) $P^{\prime} \mid P$;

(2) $O_{P} \subseteq O_{P^{\prime}}$; 
(3) existe um único e $\in \mathbb{N}^{*}$ tal que $v_{P^{\prime}}(x)=e \cdot v_{P}(x)$, qualquer que seja $x \in F$.

Além disso, se $P^{\prime} \mid P$, então $P=P^{\prime} \cap F$ e $O_{P}=O_{P^{\prime}} \cap F$.

\section{Demonstração.}

- (1) $\Longrightarrow(2)$ : Suponhamos que $P^{\prime} \mid P$ e $O_{P} \nsubseteq O_{P^{\prime}}$. Neste caso, existe $u \in O_{P}$ tal que $v_{P}(u) \geq 0$ e $v_{P^{\prime}}(u)<0$. Logo:

$$
u \notin P^{\prime} \Longrightarrow u \notin P \Longrightarrow v_{P}(u)=0 \text {. }
$$

Mostraremos que, nestas condições, existe um elemento de $P$ que não está em $P^{\prime}$, contrariando a hipótese.

Seja $t \in F, \operatorname{com} v_{P}(t)=1$. Então, $t \in P^{\prime}$ e $r:=v_{P^{\prime}}(t)>0$. Assim:

$$
v_{P}\left(u^{r} t\right)=r \cdot v_{P}(u)+v_{P}(t)=r \cdot 0+1=1,
$$

e, portanto, $u^{r} t \in P$. Mas, por outro lado, como $v_{P^{\prime}}(u) \leq-1$, temos

$$
v_{P^{\prime}}\left(u^{r} t\right)=r \cdot v_{P^{\prime}}(u)+v_{P^{\prime}}(t) \leq-r+r=0
$$

o que implica que $u^{r} t \notin P^{\prime}$.

Antes de mostrarmos que (2) $\Longrightarrow(1)$, vamos provar que

$$
\text { (*) } O_{P} \subseteq O_{P^{\prime}} \Longrightarrow O_{P}=F \cap O_{P^{\prime}} \text {. }
$$

De fato, como $O_{P^{\prime}} \cap F$ é um subanel de $F$, e $O_{P} \subseteq O_{P^{\prime}} \cap F$, temos que $O_{P}=F \cap O_{P^{\prime}}$ ou $O_{P^{\prime}} \cap F=F$ (pois $O_{P}$ é maximal em $F$ ). Suponhamos que $O_{P^{\prime}} \cap F=F$, ou seja, $F \subseteq O_{P^{\prime}}$. Tomemos $x \in F^{\prime} \backslash O_{P^{\prime}}$. Como $F^{\prime} \mid F$ é algébrica, temos que

$$
(* *) \quad x^{n}+c_{n-1} x^{n-1}+\ldots+c_{1} x+c_{0}=0,
$$

com $c_{i} \in F$ para cada $i \in\{0,1, \ldots, n-1\}$. Como $x \notin O_{P^{\prime}}$, temos então que $v_{P^{\prime}}\left(x^{n}\right)=n \cdot v_{P^{\prime}}(x)<0$. Logo,

$$
v_{P^{\prime}}\left(x^{n}\right)<v_{P^{\prime}}\left(c_{i} x^{i}\right) i=1, \ldots, n-1 .
$$

Portanto, pela desigualdade triangular estrita, temos que

$$
v_{P^{\prime}}\left(z^{n}+c_{n-1} x^{n-1}+\ldots+c_{1} x+c_{0}\right)=n \cdot v_{P^{\prime}}(x)<0 .
$$

Isso contradiz $(* *)$, e prova $(*)$.

- $(2) \Longrightarrow(1)$ : Suponha $O_{P} \subseteq O_{P^{\prime}}$. Temos então que:

$$
y \in P \Longrightarrow y^{-1} \notin O_{P} \Longrightarrow y^{-1} \notin O_{P^{\prime}} \Longrightarrow y \in P^{\prime} .
$$

Portanto, $P \subseteq P^{\prime}$. 
- $(2) \Longrightarrow(3):$ Suponha $O_{P} \subseteq O_{p^{\prime}}$. Observemos que, se $x \in F$ e $v_{P}(x)=0$, então $v_{P^{\prime}}(x)=0$ : de fato, se $u \in F$ é tal que $v_{P}(u)=0$, então $u, u^{-1} \in O_{P}$, e, portanto, $u, u^{-1} \in O_{P^{\prime}}$, o que implica $v_{P^{\prime}}(u)=0$.

Agora, seja $t \in F$ tal que $v_{P}(t)=1$, e façamos $e:=v_{P^{\prime}}(t)$. Como $P^{\prime} \mid P$, temos que $e \geq 1$. Seja então $0 \neq x \in F$ e $v_{P}(x)=: r \in \mathbb{Z}$. Logo, $v_{P}\left(x t^{-r}\right)=v_{P}(x)-r v_{P}(t)=0$. Conseqüentemente, $v_{P^{\prime}}\left(x t^{-r}\right)=0$, e assim:

$$
v_{P^{\prime}}(x)=v_{P^{\prime}}\left(x t^{-r} t^{r}\right)=v_{P^{\prime}}\left(x t^{-r}\right)+v_{P^{\prime}}\left(t^{r}\right)=r \cdot v_{P^{\prime}}(t)=e \cdot v_{P}(x)
$$

- $(3) \Longrightarrow(2)$ :

$$
x \in O_{P} \Longrightarrow v_{P}(x) \geq 0 \Longrightarrow v_{P^{\prime}}(x) \geq 0 \Longrightarrow x \in O_{P^{\prime}}
$$

Agora, é fácil ver que $P=P^{\prime} \cap F$. Seja, pois, $x \in P^{\prime} \cap F$. Como $x \in P^{\prime}$, temos $v_{P^{\prime}}(x)>0$; entretanto, por $(3)$, temos $v_{P^{\prime}}(x)=e \cdot v_{P}(x)$. Logo, $v_{P}(x)>0 \Longrightarrow x \in P$.

Uma conseqüência da proposição acima é que, se $P^{\prime} \mid P$, existe uma imersão canônica de $F_{P}=O_{P} / P$ em $F_{P^{\prime}}^{\prime}=O_{P^{\prime}} / P^{\prime}$, dada por:

$$
x(P) \longmapsto x\left(P^{\prime}\right) \text { para } x \in O_{P} .
$$

Portanto, podemos considerar $\mathbb{F}_{P}$ como subcorpo de $\mathbb{F}_{P^{\prime}}^{\prime}$ via tal imersão.

Definição 3.5. Seja $F^{\prime} / K^{\prime}$ uma extensão algébrica de $F / K$, e seja $P^{\prime} \in \mathbb{P}\left(F^{\prime}\right)$ um lugar de $F^{\prime} / K^{\prime}$ sobre $P \in \mathbb{P}(F)$.

(a) O inteiro $e\left(P^{\prime} \mid P\right):=e$, com $v_{P^{\prime}}(x)=e \cdot v_{P}(x)$ para todo $x \in F$, é chamado índice de ramificação de $P^{\prime}$ sobre $P$.

Observação: pela proposição anterior, temos que o índice de ramificação de $P^{\prime}$ sobre $P$ nada mais é que a ordem de zero em $P^{\prime}$ de um elemento primo $t \in P$.

Dizemos que $P^{\prime} \mid P$ é ramificado se $e\left(P^{\prime} \mid P\right)>1$, e que $P^{\prime} \mid P$ é não-ramificado se $e\left(P^{\prime} \mid P\right)=1$.

(b) $f\left(P^{\prime} \mid P\right):=\left[F_{P^{\prime}}^{\prime}: F_{P}\right]$ é denominado grau relativo de $P^{\prime}$ sobre $P$.

Proposição 3.6. Seja $F^{\prime} / K^{\prime}$ uma extensão algébrica de $F / K$, e $P^{\prime}$ um lugar de $F^{\prime} / K^{\prime}$ sobre $P \in \mathbb{P}(F)$. Então:

(a) $f\left(P^{\prime} \mid P\right)<\infty \Longleftrightarrow\left[F^{\prime}: F\right]<\infty$;

(b) Se $F^{\prime \prime} / K^{\prime \prime}$ é uma extensão algébrica de $F^{\prime} / K^{\prime}$, e $P^{\prime \prime} \in \mathbb{P}_{F^{\prime \prime}}$ é uma extensão de $P^{\prime}$, então:

$$
\begin{gathered}
e\left(P^{\prime \prime} \mid P\right)=e\left(P^{\prime \prime} \mid P^{\prime}\right) \cdot e\left(P^{\prime} \mid P\right) \\
f\left(P^{\prime \prime} \mid P\right)=f\left(P^{\prime \prime} \mid P^{\prime}\right) \cdot f\left(P^{\prime} \mid P\right) .
\end{gathered}
$$


Demonstração. (a) Lembrando que $\operatorname{deg}(P)=\left[F_{P}: K\right]<\infty, \operatorname{deg}\left(P^{\prime}\right)=\left[F_{P^{\prime}}^{\prime}: K^{\prime}\right]<$ $\infty$, e que $\left[F^{\prime}: F\right]<\infty \Longleftrightarrow\left[K^{\prime}: K\right]<\infty$, o diagrama abaixo prova o item (a):

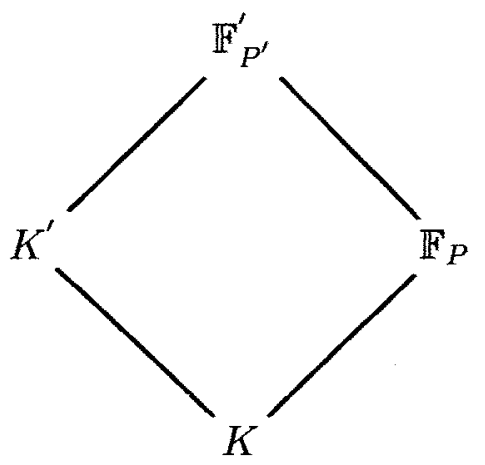

(b) Em $F \subseteq F^{\prime} \subseteq F^{\prime \prime}$, tomemos os lugares $P \subseteq P^{\prime} \subseteq P^{\prime \prime}$, e seja $t \in P$ um elemento primo. Pela definição de índice de ramificação, temos que:

$$
e\left(P^{\prime \prime} \mid P\right)=v_{P^{\prime \prime}}(t) \quad \text { e } \quad e\left(P^{\prime} \mid P\right)=v_{P^{\prime}}(t) .
$$

Como, em particular, $t \in F^{\prime}$, temos

$$
v_{P^{\prime \prime}}(t)=e\left(P^{\prime \prime} \mid P^{\prime}\right) \cdot v_{P^{\prime}}(t) \Longrightarrow e\left(P^{\prime \prime} \mid P\right)=e\left(P^{\prime \prime} \mid P^{\prime}\right) \cdot e\left(P^{\prime} \mid P\right) .
$$

Das inclusões $F_{p} \subseteq F_{P^{\prime}}^{\prime} \subseteq F_{P^{\prime \prime}}^{\prime \prime}$, segue direto que

$$
f\left(P^{\prime \prime} \mid P\right)=f\left(P^{\prime \prime} \mid P^{\prime}\right) \cdot f\left(P^{\prime} \mid P\right) \text {. }
$$

Proposição 3.7. Seja $F^{\prime} / K^{\prime}$ uma extensão algébrica de $F / K$.

(a) Para cada lugar $P^{\prime} \in \mathbb{P}\left(F^{\prime}\right)$ existe exatamente um lugar $P \in \mathbb{P}(F)$ tal que $P^{\prime} \mid P$, e este é dado por $P=P^{\prime} \cap F$.

(b) Reciprocamente, cada lugar $P \in \mathbb{P}(F)$ tem pelo menos um, porém uma quantidade finita de extensões $P^{\prime} \in \mathbb{P}\left(F^{\prime}\right)$.

Para demonstrar esta proposição usaremos a seguinte observação, demonstrada em [11] (páginas 62 e 63).

Observação 3.8. Seja $F^{\prime} / K^{\prime}$ uma extensão algébrica de $F / K$. Dados $P \in \mathbb{P}(F)$ e $P^{\prime} \in \mathbb{P}\left(F^{\prime}\right)$, temos que:

(i) existe $0 \neq z \in F$ tal que $v_{P^{\prime}}(z) \neq 0$; conseqüentemente, $P^{\prime} \cap F \neq\{0\}$;

(ii) se $x \in F / K$ tem $P$ como seu único zero, então $P^{\prime} \mid P \Longleftrightarrow v_{P^{\prime}}(x)>0$.

\section{Demonstração da proposição 3.7 .}

(a) Seja $O:=O_{P^{\prime}} \cap F$ e $P:=P^{\prime} \cap F$. 
(1) $O$ é anel de valorização de $F$ : de fato, se $z \in F \backslash O$, então $z \notin O_{P^{\prime}} \Longrightarrow z^{-1} \in O_{P^{\prime}}$; mas, $z^{-1} \in F$, e, portanto, $z^{-1} \in O$.

(2) $P:=P^{\prime} \cap F$ é o lugar associado a $O$ : de fato, se retirarmos as unidades de $O$, estaremos retirando as unidades de $O_{P^{\prime}}$ que estão em $F$, obtendo $P=P^{\prime} \cap F$, e, pelo item (i) da observação acima, teremos que $P \neq\{0\}$.

(b) Agora, dado $P \in \mathbb{P}(F)$, escolhemos $x \in F \backslash K$ tal que $P$ seja seu único zero (o que é possível, segundo a proposição 2.47). Então, pelo item (ii) da observação acima, temos que $P^{\prime} \mid P \Longleftrightarrow v_{P^{\prime}}(x)>0$. Como $x$ tem pelo menos um, porém uma quantidade finita de zeros em $F^{\prime}$, segue que $P$ tem pelo menos uma, porém uma quantidade finita de extensões $P^{\prime}$ em $F^{\prime}$.

Definição 3.9. Seja $F^{\prime} / K^{\prime}$ uma extensão algébrica de $F / K$. Dado um lugar $P \in \mathbb{P}(F)$, definimos a sua conorma (em relação à $F^{\prime} \mid F$ ) por

$$
\operatorname{Con}_{F^{\prime} / F}(P)=\sum_{P^{\prime} \mid P} e\left(P^{\prime} \mid P\right) \cdot P^{\prime} .
$$

Podemos estender a função conorma a um homomorfismo entre os grupos dos divisores de $F$ e $F^{\prime}$, fazendo:

$$
\operatorname{Con}_{F^{\prime} / F}:\left\{\begin{array}{l}
D_{F} \longrightarrow D_{F^{\prime}} \\
\sum n_{P} \cdot P \longrightarrow \sum n_{P} \cdot \operatorname{Con}_{F^{\prime} / F}(P)
\end{array}\right.
$$

Uma propriedade importante da conorma é que ela leva divisores principais de $F$ em divisores principais de $F^{\prime} \mid F$.

Proposição 3.10. Seja $F^{\prime} / K^{\prime}$ uma extensão algébrica de $F / K$. Para $0 \neq x \in F$, denotemos por $(x)^{F}$ e $(x)^{F^{\prime}}$ os divisores principais de $x$ em $D_{F}$ e $D_{F^{\prime}}$, respectivamente. Entâo, $\operatorname{Con}_{F^{\prime} / F}(x)^{F}=(x)^{F^{\prime}}$.

\section{Demonstração.}

$$
\begin{aligned}
(x)^{F^{\prime}} & =\sum_{P^{\prime} \in \mathbb{P}\left(F^{\prime}\right)} v_{P^{\prime}}(x) \cdot P^{\prime}=\sum_{P \in \mathbb{P}(F)} \sum_{P^{\prime} \mid P} e\left(P^{\prime} \mid P\right) \cdot v_{P}(x) \cdot P^{\prime} \\
& =\sum_{P \in \mathbb{P}(F)} v_{P}(x) \sum_{P^{\prime} \mid P} e\left(P^{\prime} \mid P\right) \cdot P^{\prime}=\sum_{P \in \mathbb{P}(F)} v_{P}(x) \cdot \operatorname{Con}_{F^{\prime} / F}(P) \\
& =\operatorname{Con}_{F^{\prime} / F}\left(\sum_{P \in \mathbb{P}(F)} v_{P}(x) \cdot P\right)=\operatorname{Con}_{F^{\prime} / F}\left((x)^{F}\right) .
\end{aligned}
$$


Teorema 3.11. Seja $F^{\prime} / K^{\prime}$ uma extensão finita de $F / K, P$ um lugar de $F / K$ e $P_{1}, \ldots, P_{m}$ todos os lugares de $F^{\prime} / K^{\prime}$ sobre $P$. Seja $e_{i}=e\left(P_{i} \mid P\right)$ e $f_{i}=f\left(P_{i} \mid P\right)$, com $i=1, \ldots, m$. Então:

$$
\sum_{i=1}^{m} e_{i} \cdot f_{i}=\left[F^{\prime}: F\right]
$$

Demonstração. Seja $x \in F$ tal que $P$ é seu único zero em $F / K$. Então, pelo item (ii) da observação 3.8 , os lugares $P_{1}, \ldots, P_{m}$ são exatamente os zeros de $x$ em $F^{\prime} / K^{\prime}$. A idéia é calcular $\left[F^{\prime}: K(x)\right]$ de duas formas distintas e fazer a comparação no final.

Para tanto, vamos utilizar os seguintes diagramas:
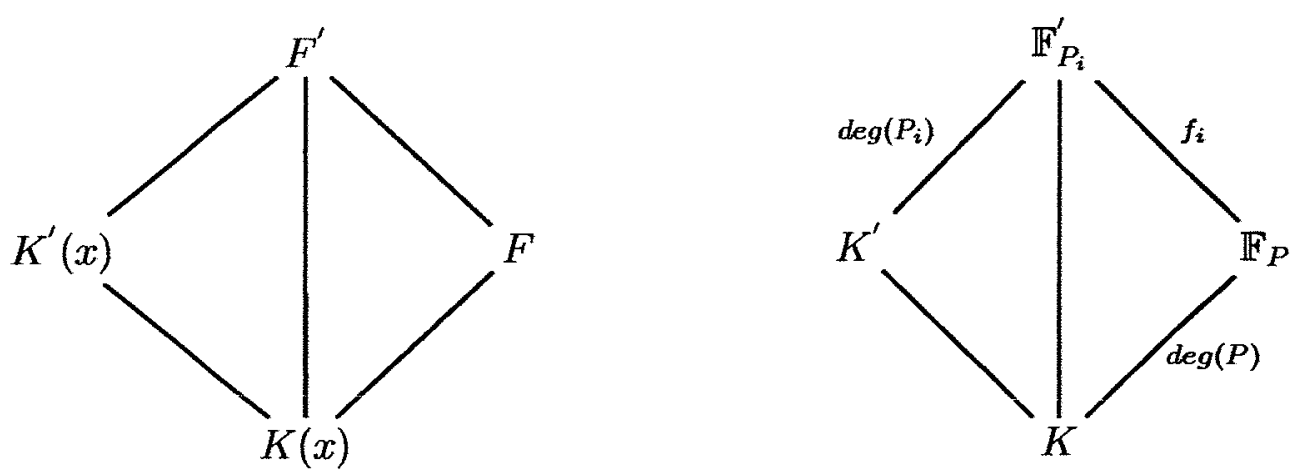

bem como os seguintes fatos:

- $\left[K^{\prime}(x): K(x)\right]=\left[K^{\prime}: K\right]$ (caso particular do lema 3.46 adiante) ;

$\left.\begin{array}{l}\text { - }\left[F^{\prime}: K^{\prime}(x)\right]=\operatorname{deg}(x)_{0}^{F^{\prime}}=\sum_{i=1}^{m} v_{P_{i}}(x) \cdot \operatorname{deg}\left(P_{i}\right) ; \\ \text { - }[F: K(x)]=\operatorname{deg}(x)_{0}^{F}=v_{P}(x) \cdot \operatorname{deg}(P) .\end{array}\right\}($ teorema 2.28)

Assim:

$$
\begin{aligned}
{\left[F^{\prime}: K(x)\right] } & =\left[F^{\prime}: K^{\prime}(x)\right] \cdot\left[K^{\prime}(x): K(x)\right] \\
& =\sum_{i=1}^{m} \underbrace{\operatorname{deg}\left(P_{i}\right) \cdot\left[K^{\prime}: K\right]}_{\underbrace{}_{P_{i}}(x)} \\
& =\sum_{i=1}^{m} \overbrace{\left(e_{i} \cdot v_{P}(x)\right)}^{v_{\left(f_{i} \cdot \operatorname{deg}(P)\right)}} \\
& =v_{P}(x) \cdot \operatorname{deg}(P) \cdot \sum_{i=1}^{m} e_{i} \cdot f_{i} .
\end{aligned}
$$

Por outro lado, temos que:

$$
\left[F^{\prime}: K(x)\right]=\left[F^{\prime}: F\right] \cdot[F: K(x)]=\left[F^{\prime}: F\right] \cdot v_{P}(x) \cdot \operatorname{deg}(P) .
$$


Fazendo a comparação, obtemos então que:

$$
\left[F^{\prime}: F\right] \cdot v_{P}(x) \cdot \operatorname{deg}(P)=v_{P}(x) \cdot \operatorname{deg}(P) \cdot \sum_{i=1}^{m} e_{i} \cdot f_{i} \Longrightarrow\left[F^{\prime}: F\right]=\sum_{i=1}^{m} e_{i} \cdot f_{i}
$$

Corolário 3.12. Seja $F^{\prime} / K^{\prime}$ uma extensão finita de $F / K$, e seja $P \in \mathbb{P}(F)$. Então:

(a) $\left|\left\{P^{\prime} \in \mathbb{P}\left(F^{\prime}\right): P \subseteq P^{\prime}\right\}\right| \leq\left[F^{\prime}: F\right]$;

(b) se $P^{\prime} \in \mathbb{P}\left(F^{\prime}\right)$ e $P^{\prime} \mid P$, então e $\left(P^{\prime} \mid P\right) \leq\left[F^{\prime}: F\right]$ e $f\left(P^{\prime} \mid P\right) \leq\left[F^{\prime}: F\right]$.

Demonstração. Imediata.

Corolário 3.13. Seja $F^{\prime} / K^{\prime}$ uma extensão finita de $F / K$. Então, dado $A \in D_{F}$, temos:

$$
\operatorname{deg}\left(\operatorname{Con}_{F^{\prime} / F}(A)\right)=\frac{\left[F^{\prime}: F\right]}{\left[K^{\prime}: K\right]} \cdot \operatorname{deg}(A)
$$

Demonstração. É suficiente supor que $A$ é um divisor primo, isto é, $A=P \in \mathbb{P}(F)$. Assim:

$$
\begin{aligned}
\operatorname{deg}\left(\operatorname{Con}_{F^{\prime} / F}(P)\right) & =\operatorname{deg}\left(\sum_{P^{\prime} \mid P} e\left(P^{\prime} \mid P\right) \cdot P^{\prime}\right)=\sum_{P^{\prime} \mid P} e\left(P^{\prime} \mid P\right) \cdot \operatorname{deg}\left(P^{\prime}\right) \\
& =\sum_{P^{\prime} \mid P} e\left(P^{\prime} \mid P\right) \cdot f\left(P^{\prime} \mid P\right) \cdot \frac{\operatorname{deg}(P)}{\left[K^{\prime}: K\right]}=\frac{\left[F^{\prime}: F\right]}{\left[K^{\prime}: K\right]} \cdot \operatorname{deg}(P) .
\end{aligned}
$$

Proposição 3.14. Considere um corpo de funçôes $F / K$ e um polinômio

$$
\varphi(T)=a_{n} T^{n}+a_{n-1} T^{n-1}+\ldots+a_{1} T+a_{0},
$$

onde $a_{i} \in F$. Se existe $P \in \mathbb{P}(F)$ tal que $v_{P}\left(a_{n}\right)=0, v_{P}\left(a_{i}\right) \geq 0$ para $i=1, \ldots, n-1$, $v_{P}\left(a_{0}\right)<0$ e mdc $\left(n ; v_{P}\left(a_{0}\right)\right)=1$, então $\varphi(T)$ é irredutivel em $F[T]$. Se $F^{\prime}=F(y)$, onde y é raiz de $\varphi(T)$, então $P \in \mathbb{P}(F)$ tem uma única extensão $P^{\prime} \in \mathbb{P}\left(F^{\prime}\right)$, e temos $e\left(P^{\prime} \mid P\right)=n$ e $f\left(P^{\prime} \mid P\right)=1$.

Demonstração. Considere a extensão de corpos $F^{\prime}=F(y)$, onde $\varphi(y)=0$. O grau de $F^{\prime} \mid F$ é $\left[F^{\prime}: F\right] \leq \operatorname{deg} \varphi(T)=n$. Valendo a igualdade se, e somente se, $\varphi(T)$ é irredutível em $F[T]$, tomemos $P^{\prime} \in \mathbb{P}\left(F^{\prime}\right)$ com $P^{\prime} \mid P$. Como $\varphi(y)=0$, temos

$$
-a_{n} y^{n}=a_{0}+a_{1} y+\ldots+a_{n-1} y^{n-1} \text {. }
$$


Decorre das hipóteses que $v_{P^{\prime}}\left(a_{n}\right)=0, v_{P^{\prime}}\left(a_{i}\right) \geq 0$ para $i=1, \ldots, n-1$, e $v_{P^{\prime}}\left(a_{0}\right)<0$. É fácil ver que $v_{P^{\prime}}(y)<0$ : de fato, se $v_{P^{\prime}}(y) \geq 0$, teríamos $v_{p^{\prime}}\left(a_{i} y\right) \geq 0$ para $i=1, \ldots, n-1$, e assim:

$$
v_{P^{\prime}}\left(a_{0}+a_{1} y+\ldots+a_{n-1} y^{n-1}\right)=v_{P^{\prime}}\left(-a_{n} y^{n}\right) \geq 0 \Longrightarrow v_{P^{\prime}}\left(a_{0}\right) \geq 0
$$

o que seria uma contradição.

Agora, sendo $v_{P^{\prime}}(y)<0$, e como $v_{P^{\prime}}\left(a_{i}\right) \geq 0$ para $i=1, \ldots, n-1$, temos

$$
v_{P^{\prime}}\left(y^{n}\right)<v_{P^{\prime}}\left(a_{i} y^{i}\right) \Longrightarrow v_{P^{\prime}}\left(y^{n}\right)<v_{P^{\prime}}\left(a_{1} y+\ldots+a_{n-1} y^{n-1}\right) \text {. }
$$

Por outro lado, temos que:

$$
v_{P^{\prime}}\left(y^{n}\right) \geq \min \left\{v_{P^{\prime}}\left(a_{0}\right), v_{P^{\prime}}\left(a_{1} y+\ldots+a_{n-1} y^{n-1}\right)\right\}
$$

Portanto, $v_{P^{\prime}}\left(y^{n}\right)=v_{P^{\prime}}\left(a_{0}\right)$. Fazendo $e:=e\left(P^{\prime} \mid P\right)$, temos

$$
n \cdot v_{P^{\prime}}(y)=e\left(P^{\prime} \mid P\right) \cdot v_{P}\left(a_{0}\right)
$$

Mas, como $m d c\left(n, v_{P}\left(a_{0}\right)\right)=1$, temos que $n \mid e$, e portanto

$$
n \leq e \Longrightarrow e \geq\left[F^{\prime} \mid F\right] \text {. }
$$

Agora, usando a igualdade fundamental $\sum e_{i} f_{i}=\left[F^{\prime}: F\right]$ (teorema 3.11), seguem os resultados desejados.

\subsection{Subanéis de Corpos de Funções}

A idéia principal desta seção é apresentar alguns conceitos e resultados sobre anéis de holomorfia de um corpo de funções algébricas. Tal conteúdo servirá de base para a seção seguinte.

Definição 3.15. Um subanel de $F / K$ é um anel $R$ tal que $K \subseteq R \subseteq F$ e $R$ não é corpo.

Logo, se $R$ é um anel de $F / K$, então $K \subsetneq R \subsetneq F$. Temos que, se $P \in \mathbb{P}(F)$, então $O_{P}$ é um exemplo trivial de subanel de $F / K$.

Definição 3.16. Para $\varnothing \neq S \subsetneq \mathbb{P}(F)$, definimos

$$
O_{S}:=\bigcap_{P \in S} O_{P}
$$

e qualquer anel $R \subseteq F$ da forma acima será chamado anel de holomorfia de $F / K$.

Lema 3.17. (a) Todo anel de valorização $O_{P}$ é um anel de holomorfia, onde $O_{P}=O_{S}$, com $S=\{P\}$. 
(b) Todo anel de holomorfia $O_{S}$ é um subanel de $F / K$.

(c) Para $P \in \mathbb{P}(F)$ e $\varnothing \neq S \subsetneq \mathbb{P}(F)$, temos $O_{S} \subseteq O_{P} \Longleftrightarrow P \in S$. Conseqüentemente, $O_{S}=O_{T} \Longleftrightarrow S=T$.

Demonstração. (a) Óbvio.

(b) Como $O_{S}$ é um anel com $K \subseteq O_{S} \subseteq F$, é suficiente mostrar que $O_{S}$ não é corpo.

Seja $P_{1} \in S$. Como $S \neq \mathbb{P}(F)$, pelo teorema da aproximação forte, existe $0 \neq x \in F$ tal que

$$
v_{P_{1}}(x)>0 \text { e } v_{P}(x) \geq 0, \forall P \in S \backslash\left\{P_{1}\right\}
$$

Em particular, $x \in O_{S}$. Conseqüentemente,

$$
v_{P_{1}}\left(x^{-1}\right)<0 \Longrightarrow x^{-1} \notin O_{P_{1}} \Longrightarrow x^{-1} \notin O_{S} \text {. }
$$

(c) É óbvio que $P \in S \Longrightarrow O_{S} \subseteq O_{P}$. Provaremos que $P \notin S \Longrightarrow O_{S} \nsubseteq O_{P}$.

Se $P \notin S$, pelo teorema da aproximação forte, podemos tomar $z \in F$ tal que

$$
\text { (1) } v_{P}(z)<0 \text { e } v_{Q}(z) \geq 0, \forall Q \in S
$$

ou seja, $z \in O_{S}$, mas $z \notin O_{P}$. (1) é facilmente aceitável caso tenhamos $S \cup\{P\} \neq$ $\mathbb{P}(F)$. Por outro lado, se $S \cup\{P\}=\mathbb{P}(F)$, basta tomarmos $z \in O_{S}$ transcendente sobre $K$. Como $z$ tem pelo menos um pólo, este será necessariamente $P$, ou seja, (1) continua valendo.

Agora, é claro que $S=T \Longrightarrow O_{S}=O_{T}$. Provaremos que $O_{S}=O_{T} \Longrightarrow S=T$.

$$
P \in S \Longrightarrow O_{S} \subseteq O_{P} \Longrightarrow O_{T} \subseteq O_{P} \Longrightarrow P \in T \text {. }
$$

Portanto, $S \subseteq T$, e analogamente temos $T \subseteq S$.

Definição 3.18. Seja $R$ um subanel de $F / K$.

(a) Um elemento $z \in F$ é inteiro sobre $R$ se $f(z)=0$ para algum polinômio mônico $f(x) \in R[x]$.

(b) O conjunto $i c_{F}(R):=\{z \in F: z$ é inteiro sobre $R\}$ é chamado fecho inteiro de $R$ em $F$.

(c) Seja $F_{0} \subseteq F$ o corpo quociente de $R$. $R$ é dito integralmente fechado se $i c_{F_{0}}(R)=$ $R$.

Proposição 3.19. Seja $O_{S}$ um anel de holomorfia de $F / K$. Então:

(a) F é o corpo quociente de $O_{S}$;

(b) $O_{S}$ é integralmente fechado. 
Observação: Em particular, um anel de valorização possui tais propriedades.

Demonstração. (a) Seja $0 \neq x \in F$. Como $x$ tem um número finito de zeros e pólos, temos, pelo teorema 2.46 (da aproximação forte), que existe $0 \neq z \in F$ tal que:

$$
v_{P}(z) \geq \max \left\{0, v_{P}\left(x^{-1}\right)\right\} \quad, \quad \forall P \in S .
$$

Então, como $v_{P}(z) \geq 0, \forall P \in S$, temos que $z \in O_{S}$. Seja $y:=z x$. Assim,

$$
v_{P}(y)=v_{P}(z)+v_{P}(x) \geq v_{P}\left(x^{-1}\right)+v_{P}(x)=0, \forall P \in S .
$$

Logo, $y \in O_{S}$, e, portanto, $x=y z^{-1}$ está no corpo quociente de $O_{S}$.

(b) Seja $u \in F$ inteiro sobre $O_{S}$. Então

$$
u^{n}+a_{n-1} u^{n-1}+\ldots+a_{1} u+a_{0}=0
$$

onde $a_{i} \in O_{S}$ para cada $i=0, \ldots, n-1$. Mostraremos que $u \in O_{S}$. Suponhamos que $u \notin O_{S}$. Então, existe $P \in S$ tal que $v_{P}(u)<0$. Como $v_{P}\left(a_{i}\right) \geq 0$, temos que $v_{P}\left(u^{n}\right)<v_{P}\left(a_{i} u^{i}\right)$ para todo $i=0,1, \ldots, n-1$. Logo,

$$
v_{P}\left(u^{n}+a_{n-1} u^{n-1}+\ldots+a_{0}\right)=v_{P}\left(u^{n}\right)<0 \Longrightarrow v_{P}(0)<0,
$$

o que é absurdo.

O próximo teorema nos diz que, se $R$ é subanel de $F / K$, então o fecho inteiro de $R$ em $F$ é um anel de holomorfia.

Teorema 3.20. Seja $R$ um subanel de $F / K$, e $S(R)=\left\{P \in \mathbb{P}(F): R \subseteq O_{P}\right\}$. Então:

(a) $\varnothing \neq S(R) \subsetneq \mathbb{P}(F)$;

(b) o fecho inteiro de $R$ em $F$ é $i c_{F}(R)=O_{S(R)}$; em particular, ic $c_{F}(R)$ é um subanel integralmente fechado de $F / K$ com corpo quociente $F$.

Demonstração. (a) Como $R$ não é corpo, podemos encontrar um ideal próprio $\{0\} \neq$ $I \subsetneq R$, e, pelo teorema 2.16, existe um lugar $P \in \mathbb{P}(F)$ tal que $I \subseteq P$ e $R \subseteq O_{P}$. Portanto, $S(R) \neq \varnothing$. Por outro lado, tomamos $x \in R$ transcendente sobre $K$ (este existe, pois $K \subsetneq R$ ). Então existe $P \in \mathbb{P}(F)$ tal que $v_{P}(x)<0$. Logo, $P \notin S(R)$, e conseqüentemente $S(R) \subsetneq \mathbb{P}(F)$.

(b) Como

$$
O_{S(R)}=\bigcap_{P \in S(R)} O_{P}
$$

temos que $R \subseteq O_{S(R)}$, e, como $O_{S(R)}$ é integralmente fechado, temos que $i c_{F}(R) \subseteq$ $O_{S(R)}$. Para provar a outra inclusão, tomemos $z \in O_{S(R)}$, e assumiremos o seguinte fato (vide [11], página 69):

$$
z^{-1} \cdot R\left[z^{-1}\right]=R\left[z^{-1}\right]
$$


Do fato acima, temos que existem $s \in \mathbb{N}$ e $a_{i} \in R$, com $i=0,1, \ldots, s$, tais que vale a seguinte relação:

$$
z^{-1} \cdot \sum_{i=0}^{s} a_{i}\left(z^{-1}\right)^{i}=1
$$

Multiplicando por $z^{s+1}$, temos

$$
z^{s+1}-\sum_{i=0}^{s} a_{i} z^{s-i}=0 .
$$

Portanto, $z$ é inteiro sobre $R$.

Corolário 3.21. Um subanel $R$ de $F / K$, com corpo quociente $F$, é integralmente fechado se, e somente se, $R$ é anel de holomorfia.

Demonstração. Na hipótese de $R$ ser de holomorfia, o resultado segue da proposição 3.19. Na recíproca, temos $i c_{F}(R)=R$. Logo, $R=O_{S(R)}$ (pelo teorema 3.20), e, portanto, $R$ é de holomorfia.

Proposição 3.22 ([11], página 71). Se $\varnothing \neq S \subseteq \mathbb{P}(F)$ é um conjunto finito de lugares de $F / K$, então $O_{S}$ é um domínio de ideais principais.

\subsection{Base Local Integral}

Nesta seção, estudaremos o fecho inteiro de um subanel $R$ de $F / K$ em uma extensão finita $F^{\prime}$ de $F$, apresentaremos o conceito de base local integral, e finalizaremos apresentando o teorema de Kummer, que será bastante utilizado no Capítulo 4.

Aqui, assumiremos $F^{\prime} / F$ finita e separável.

Proposição 3.23. Seja $R$ um subanel integralmente fechado de $F / K$ (isto é, $R$ é um subanel de holomorfia de $F / K)$. Para $z \in F^{\prime}$, seja $\varphi(T) \in F[T]$ o seu polinômio minimal sobre F. Então:

$$
z \text { é inteiro sobre } R \Longleftrightarrow \varphi(T) \in R[T] \text {. }
$$

Demonstração. É óbvio que

$$
\varphi(T) \in R[T] \Longrightarrow z \text { é inteiro sobre } R \text {. }
$$

A recíproca não é evidente, pois, a priori, $\varphi(T)$ é um polinômio muito específico. Vejamos então tal demonstração.

Tomamos $f(T) \in R[T]$ tal que $f(z)=0$. Como $\varphi(T)$ é o polinômio minimal de $z$ sobre $F$, temos que existe um polinômio $\psi(T) \in F[T]$ tal que $f(T)=\varphi(T) \cdot \psi(T)$. Seja $F^{\prime \prime} \supseteq F^{\prime}$ uma extensão finita de $F$ contendo todas as raízes de $\varphi(T)$ e $R^{\prime \prime}=i c_{F^{\prime \prime}}(R)$, isto é, o fecho inteiro de $R$ em $F^{\prime \prime}$. Como todas as raízes de $\varphi(T)$ são raízes de $f(T) \in R[T]$, temos que tais raízes estão em $R^{\prime \prime}$. Como os coeficientes de $\varphi(T)$ são expressões polinomiais de suas raízes, temos que $\varphi(T) \in R^{\prime \prime}[T]$. Logo, $\varphi(T) \in F[T] \cap R^{\prime \prime}[T]$. Mas, como $R$ é integralmente fechado em $F$, temos que $F \cap R^{\prime \prime}=R$. Portanto, $\varphi(T) \in R[T]$. 
Corolário 3.24. Utilizando a notação da proposição anterior, seja $T_{F_{F^{\prime} \mid F}}: F^{\prime} \longrightarrow F a$ função traço de $F^{\prime}$ para $F$, e $x \in F^{\prime}$ um inteiro sobre $R$. Então, $T_{r_{F^{\prime} \mid F}}(x) \in R$.

Demonstração. Como $x$ é inteiro sobre $R$ segue, pela proposição anterior, que o polinômio minimal de $x$ sobre $F$ está em $R[T]$. Mas, pelas propriedades da função traço, sabemos que $T_{r_{F^{\prime} \mid F}}(x)$ é um múltiplo natural de um dos coeficientes do polinômio minimal de $x$. Logo, $T_{r_{F^{\prime} \mid F}}(x) \in R$. 73).

As demonstrações dos dois próximos resultados podem ser encontradas em [11] (página

Proposição 3.25. Seja $M \mid L$ uma extensão finita e separável, e $\left\{z_{1}, \ldots, z_{n}\right\}$ uma base de $M \mid L$. Então existem únicos $z_{1}^{*}, \ldots, z_{n}^{*} \in M$ tais que

$$
T_{r_{M \mid L}}\left(z_{i} z_{j}^{*}\right)=\delta_{i j}
$$

onde $\delta_{i j}$ denota o símbolo de Kronecker. $O$ conjunto $\left\{z_{1}^{*}, \ldots, z_{n}^{*}\right\}$ também é base de $M \mid L$, chamado base dual de $\left\{z_{1}, \ldots, z_{n}\right\}$ (relativa ao traço).

Teorema 3.26. Seja $R$ um subanel integralmente fechado de $F \mid K$, com corpo quociente $F$, e $F^{\prime} \mid F$ uma extensão finita e separável de grau $n$. Seja $R^{\prime}=i c_{F^{\prime}}(R)$ o fecho inteiro de $R$ em $F^{\prime}$. Então:

(a) existe uma base de $F^{\prime} \mid F$ contida em $R^{\prime}$;

(b) se $\left\{z_{1}, \ldots, z_{n}\right\} \subseteq R^{\prime}$ é uma base de $F^{\prime} \mid F$ e $\left\{z_{1}^{*}, \ldots, z_{n}^{*}\right\}$ denota a base dual com respeito à função traço, então:

$$
\sum_{i=1}^{n} R z_{i} \subseteq R^{\prime} \subseteq \sum_{i=1}^{n} R z_{i}^{*}
$$

(c) na hipótese adicional de $R$ ser um domínio de ideais principais, então existe uma base $\left\{u_{1}, \ldots, u_{n}\right\}$ de $F^{\prime} \mid F$ com a propriedade

$$
R^{\prime}=\sum_{i=1}^{n} R u_{i}
$$

Corolário 3.27. Seja $F^{\prime} \mid F$ uma extensão finita e separável de $F \mid K$, eP $P \mathbb{P}(F)$ um lugar de $F / K$. Então, o fecho inieiro de $O_{P}$ em $F^{\prime}$ (denotado por $O_{P}^{\prime}$ ) é dado por:

$$
O_{P}^{\prime}=\bigcap_{P^{\prime} \mid P} O_{P^{\prime}}
$$


e existe uma base $\left\{u_{1}, \ldots, u_{n}\right\}$ de $F^{\prime} \mid F$ tal que

$$
O_{P^{\prime}}=\sum_{i=1}^{n} O_{P} \cdot u_{i}
$$

Observação: tal base é denominada base integral de $O_{P^{\prime}}$ sobre $O_{P}$ (ou base local integral de $F^{\prime} \mid F$ para o lugar $P$ ).

Demonstração. Façamos $R:=O_{P}$, e consideremos

$$
S(R)=\left\{P^{\prime} \in \mathbb{P}\left(F^{\prime}\right): R \subseteq O_{P^{\prime}}\right\}=\left\{P^{\prime} \in \mathbb{P}\left(F^{\prime}\right): P^{\prime} \mid P\right\}
$$

Por definição, temos que

$$
O_{S(R)}=\bigcap_{P^{\prime} \in S(R)} O_{P^{\prime}}=\bigcap_{P^{\prime} \mid P} O_{P^{\prime}}
$$

Mas, pelo item (b) do teorema 3.20 , temos que $i c_{F^{\prime}}(R)=O_{S(R)}$, ou seja,

$$
O_{P}^{\prime}=O_{S(R)} \Longrightarrow O_{P}^{\prime}=\bigcap_{P^{\prime} \mid P} O_{P^{\prime}}
$$

Agora, como $O_{P}$ é um domínio de ideais principais, temos, pelo item (c) do teorema anterior, que existe uma base $\left\{u_{1}, \ldots, u_{n}\right\}$ de $F^{\prime} \mid F$ tal que:

$$
O_{P}^{\prime}=\sum_{i=1}^{n} O_{P} \cdot u_{i}
$$

Teorema 3.28. Seja $F / K$ um corpo de funçôes, e $F^{\prime} / F$ uma extensão finita e separável. Então, qualquer base $\left\{z_{1}, \ldots, z_{n}\right\}$ é base local integral para quase todos os lugares $P \in$ $\mathbb{P}(F)$.

Demonstração. Consideremos $\left\{z_{1}^{*}, \ldots, z_{n}^{*}\right\}$ base dual de $\left\{z_{1}, \ldots, z_{n}\right\}$. Os polinômios minimais de $z_{1}, \ldots, z_{n}, z_{1}^{*}, \ldots, z_{n}^{*}$ sobre $F$ envolvem apenas uma quantidade finita de coeficientes. Seja $S \subseteq \mathbb{P}(F)$ o conjunto de todos os pólos desses coeficientes. $S$ é finito, e, se $P \notin S$, então $z_{1}, \ldots, z_{n}, z_{1}^{*}, \ldots, z_{n}^{*} \in i c_{F^{\prime}}\left(O_{P}\right)=O_{P}^{\prime}$, isto é,

$$
\left\{z_{1}, \ldots, z_{n}\right\} \subseteq O_{P}^{\prime} \quad \text { e } \quad\left\{z_{1}^{*}, \ldots, z_{n}^{*}\right\} \subseteq O_{P}^{\prime}
$$

Como $O_{P}^{\prime}=i c_{F^{\prime}}\left(O_{P}\right)$, pelo item (b) do teorema 3.26, temos que:

$$
\sum_{i=1}^{n} O_{P} z_{i} \subseteq O_{P}^{\prime} \subseteq \sum_{i=1}^{n} O_{P} z_{i}^{*} \quad \text { e } \quad \sum_{i=1}^{n} O_{P} z_{i} \supseteq O_{P}^{\prime} \supseteq \sum_{i=1}^{n} O_{P} z_{i}^{*}
$$

Portanto,

$$
\sum_{i=1}^{n} O_{P} z_{i}=O_{P}^{\prime}=\sum_{i=1}^{n} O_{P} z_{i}^{*}
$$

Assim, $\left\{z_{1}, \ldots, z_{n}\right\}$ é base integral para qualquer $P \notin S$. Logo, $\left\{z_{1}, \ldots, z_{n}\right\}$ e $\left\{z_{1}^{*}, \ldots, z_{n}^{*}\right\}$ são bases integrais para quase todos os lugares $P \in \mathbb{P}(F)$. 
Comentário: $\mathrm{O}$ corolário 3.27 nos diz que, dado $P \in \mathbb{P}(F)$, existe uma base integral de $F^{\prime} / F$ para $P$. O teorema anterior, em particular, nos diz que, dada a base de $F^{\prime} / F$, existe $P \in \mathbb{P}(F)$ para o qual tal base é integral.

O próximo teorema nos fornece um método para determinar as extensões de um lugar $P \in \mathbb{P}(F)$ em $F^{\prime}$, e será fortemente usado no Capítulo 4.

Usaremos as seguintes notações:

- $\bar{F}:=\mathbb{F}_{P}$ é o corpo residual de $P$;

- $\bar{a}:=a(P) \in \bar{F}$ é a classe de resíduos de $a \in O_{P}$;

- se $\psi(T)=\sum c_{i} T^{i}$ é um polinômio com coeficientes $c_{i} \in O_{P}$, denotaremos por $\bar{\psi}(T)=$ $\sum \bar{c}_{i} T^{i} \in \bar{F}[T]$, a imagem de $\psi(T)$ em $\bar{F}[T]$.

Teorema 3.29 (Teorema de Kummer). Seja $F^{\prime}=F(y)$, onde y é inteiro sobre $O_{P}, e$ considere o seu polinômio minimal $\varphi(T) \in O_{P}[T]$ sobre $F$. Seja

$$
\bar{\varphi}(T)=\prod_{i=1}^{r} \gamma_{i}(T)^{\epsilon_{i}}
$$

a decomposição de $\bar{\varphi}(T)$ em fatores irredutíveis sobre $\bar{F}$ (isto é, polinômios $\gamma_{1}, \gamma_{2}, \ldots, \gamma_{r}$ irredutiveis, mônicos, dois a dois distintos em $\bar{F}[T]$ e $\left.\epsilon_{i} \geq 1\right)$. Escolhendo polinômios mônicos $\varphi_{i}(T) \in O_{P}[T]$, com $\bar{\varphi}_{i}(T)=\gamma_{i}(T)$ e $\operatorname{deg}\left(\bar{\varphi}_{i}(T)\right)=\operatorname{deg}\left(\gamma_{i}(T)\right)$, temos:

(a) para $1 \leq i \leq r$, existem lugares $P_{i} \in \mathbb{P}\left(F^{\prime}\right)$ satisfazendo

$$
P_{i} \mid P, \varphi_{i}(y) \in P_{i}, \quad f\left(P_{i} \mid P\right) \geq \operatorname{deg}\left(\gamma_{i}(T)\right) \text { e ainda } i \neq j \Longrightarrow P_{i} \neq P_{j}
$$

(b) supondo, adicionalmente, a validade de uma das seguintes hipóteses

$$
\epsilon_{i}=1 \text { para } i=1, \ldots, r
$$

ou

$$
\left\{1, y, y^{2}, \ldots, y^{n-1}\right\} \text { é uma base integral para } P(* *)
$$

temos que:

- para cada $1 \leq i \leq r$, existe um único lugar $P_{i} \in \mathbb{P}(F)$ com $P_{i} \mid P$ e $\varphi_{i}(P) \in P_{i}$;

- os lugares $P_{1}, \ldots, P_{r}$ são todos os lugares de $F^{r}$ sobre $P$, e teremos

$$
\operatorname{Con}_{F^{\prime} / F}(P)=\sum_{i=1}^{r} \epsilon_{i} P_{i},
$$

ou seja, $e\left(P_{i} \mid P\right)=\epsilon_{i}$;

- o corpo residual $F_{P_{i}}^{\prime}=O_{P_{i}} / P_{i}$ é isomorfo à $\bar{F}[T] /<\gamma_{i}(T)>$, e, portanto, $f\left(P_{i} \mid P\right)=\operatorname{deg}\left(\gamma_{i}(T)\right)$. 
Demonstração. (a) Seja $\bar{F}_{i}:=\bar{F}[T] /\left\langle\gamma_{i}(T)\right\rangle$. Como $\gamma_{i}(T)$ é irredutível sobre $\bar{F}$, temos que $\bar{F}_{i}$ é uma extensão de $\bar{F}$ de grau

$$
\left[\bar{F}_{i}: \bar{F}\right]=\operatorname{deg}\left(\gamma_{i}(T)\right)
$$

Considerando o anel

$$
O_{P}[y]=\sum_{j=0}^{n-1} O_{P} y^{j},
$$

onde $n=\operatorname{deg}(\varphi(T))=\left[F^{\prime}: F\right]$, é possível mostrar ([11], página 77) que, para cada $1 \leq i \leq r$, a função

$$
\sigma_{i}=\left\{\begin{array}{l}
O_{P}[y] \longrightarrow \bar{F}_{i} \\
\sum_{j=0}^{n-1} c_{j} y^{j} \longmapsto \sum_{j=0}^{n-1} \bar{c}_{j} T^{j} \bmod \gamma_{i}(T)
\end{array}\right.
$$

é um epimorfismo, cujo kernel é dado por

$$
\operatorname{ker}\left(\sigma_{i}\right)=P \cdot O_{P}[y]+\varphi_{i}(y) \cdot O_{P}[y] .
$$

Como $\{0\} \neq \operatorname{ker}\left(\sigma_{i}\right) \subsetneq O_{P}[y]$ é um ideal de $O_{P}[y]$, e este é subanel de $F^{\prime}$, temos, pelo teorema 2.16, que existe um lugar $P_{i} \in \mathbb{P}\left(F^{\prime}\right)$ tal que $k e r\left(\sigma_{i}\right) \subseteq P_{i}$ e $O_{P}[y] \subseteq O_{P_{i}}$, de onde concluímos que

- $O_{P} \subseteq O_{P_{i}} \Longrightarrow P_{i} \mid P$;

- $P \cdot O_{P}[y]+\varphi_{i}(y) \cdot O_{P}[y] \subseteq P_{i} \Longrightarrow \varphi_{i}(y) \in P_{i}$.

Como $O_{P}[y] / \operatorname{ker}\left(\sigma_{i}\right) \subseteq O_{P_{i}} / P_{i}=\mathbb{F}_{P_{i}}$ e $O_{P}[y] / \operatorname{ker}\left(\sigma_{i}\right) \simeq \bar{F}_{i}$ (via $\sigma_{i}$ ), temos o seguinte diagrama:

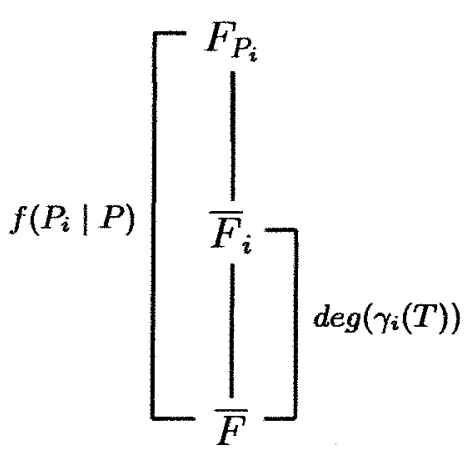

Assim, concluímos que:

$$
f\left(P_{i} \mid P\right) \geq \operatorname{deg}\left(\gamma_{i}(T)\right) \text {. }
$$

Agora, mostraremos que $i \neq j \Longrightarrow P_{i} \neq P_{j}$. Sendo $i \neq j$, temos que $\gamma_{i}(T)=\bar{\varphi}_{i}(T)$ e $\gamma_{j}(T)=\bar{\varphi}_{j}(T)$, que são primos entre si em $\bar{F}[T]$. Então, existe $\lambda_{i}(T), \lambda_{j}(T) \in O_{P}[T]$ tais que

$$
\bar{\varphi}_{i}(T) \cdot \bar{\lambda}_{i}(T)+\bar{\varphi}_{j}(T) \cdot \bar{\lambda}_{j}(T)=1 \Longrightarrow \varphi_{i}(y) \cdot \lambda_{i}(y)+\varphi_{j}(y) \cdot \lambda_{j}(y)-1 \in P \cdot O_{P}[y]
$$


Como

$$
k e r\left(\sigma_{i}\right)+k e r\left(\sigma_{j}\right)=P \cdot O_{P}[y]+\varphi_{i}(y) \cdot O_{P}[y]+\varphi_{j}(y) \cdot O_{P}[y],
$$

temos que $1 \in \operatorname{ker}\left(\sigma_{i}\right)+\operatorname{ker}\left(\sigma_{j}\right)$. Como $k \operatorname{ker}\left(\sigma_{i}\right) \subseteq P_{i}$ e $k e r\left(\sigma_{j}\right) \subseteq P_{j}$, temos que

$$
P_{i} \neq P_{j}
$$

pois, se $P_{i}=P_{j}$, temos $1 \in P_{i}$, o que é absurdo.

(b) Suponhamos agora que valha $(*)$, isto é,

$$
\bar{\varphi}(T)=\prod_{i=1}^{r} \gamma_{i}(T)
$$

Como $\varphi(T)$ é mônico, temos $\operatorname{deg}(\varphi(T))=\operatorname{deg}(\bar{\varphi}(T))$, e, portanto,

$$
\begin{aligned}
{\left[F^{\prime}: F\right]=\operatorname{deg}(\varphi(T)) } & =\sum_{i=1}^{r} \operatorname{deg}\left(\gamma_{i}(T)\right) \leq \sum_{i=1}^{r} f\left(P_{i} \mid P\right) \\
& \leq \sum_{i=1}^{r} f\left(P_{i} \mid P\right) e\left(P_{i} \mid P\right) \leq \sum_{P^{\prime} \mid P} e\left(P^{\prime} \mid P\right) f\left(P^{\prime} \mid P\right) \\
& =\left[F^{\prime}: F\right]
\end{aligned}
$$

ou seja,

$$
\sum_{i=1}^{r} \operatorname{deg}\left(\gamma_{i}(T)\right)=\sum_{i=1}^{r} f\left(P_{i} \mid P\right)=\sum_{i=1}^{r} f\left(P_{i} \mid P\right) e\left(P_{i} \mid P\right)=\sum_{i=1}^{r} e\left(P^{\prime} \mid P\right) f\left(P^{\prime} \mid P\right)
$$

e, conseqüentemente, temos:

- (I) $f\left(P_{i} \mid P\right)=\operatorname{deg}\left(\gamma_{i}(T)\right)$, pois, se existisse $i \in\{1, \ldots, r\}$ tal que $f\left(P_{i} \mid P\right)>$ $\operatorname{deg}\left(\gamma_{i}(T)\right)$, teríamos

$$
\sum_{i=1}^{r} \operatorname{deg}\left(\gamma_{i}(T)\right)<\sum_{i=1}^{r} f\left(P_{i} \mid P\right)
$$

- (II) $e\left(P_{i} \mid P\right)=\epsilon_{i}=1$, pois, se existisse $i \in\{1, \ldots, r\}$ tal que $e\left(P_{i} \mid P\right)>1$, teríamos

$$
\sum_{i=1}^{r} f\left(P_{i} \mid P\right)<\sum_{i=1}^{r} f\left(P_{i} \mid P\right) e\left(P_{i} \mid P\right)
$$

- (III) os únicos lugares $P^{\prime}$ de $\mathbb{P}\left(F^{\prime}\right)$ tais que $P^{\prime} \mid P$ são $P_{1}, P_{2}, \ldots, P_{r}$, pois, se existisse $P^{\prime} \in \mathbb{P}\left(F^{\prime}\right)$ tal que $P^{\prime} \neq P_{i}$ para todo $i \in\{1, \ldots, r\}$, e $P^{\prime} \mid P$, teríamos

$$
\sum_{i=1}^{r} e\left(P_{i} \mid P\right) f\left(P_{i} \mid P\right)<\sum_{i=1}^{r} e\left(P^{\prime} \mid P\right) f\left(P^{\prime} \mid P\right) \text {. }
$$

Omitiremos a demonstração quando da validade da segunda hipótese, pois esta não será usada no Capítulo 4. 


\subsection{O Cotraço das Diferenciais de Weil e a Fórmula do Gênero de Hurwitz}

Nesta seção, consideraremos a seguinte situação: $F / K$ corpo de funções algébricas, $F^{\prime} / F$ uma extensão finita e separável, $K^{\prime}$ o corpo das constantes de $F^{\prime}$. Como $K$ é perfeito, temos que $K^{\prime} / K$ é finita e separável.

Inicialmente, associaremos uma diferencial de Weil em $F / K$ com uma diferencial de Weil em $F^{\prime} / F$. A partir desta associação, conseguiremos obter uma fórmula que relaciona o gênero de $F / K$ com o gênero de $F^{\prime} / K^{\prime}$. Tal fórmula será de grande utilidade no Capítulo 4.

Definição 3.30. Para $P \in \mathbb{P}(F)$, seja $O_{P}^{\prime}:=i c_{F^{\prime}}\left(O_{P}\right)$ o fecho inteiro de $O_{P}$ em $F^{\prime}$. Então, o conjunto:

$$
\mathfrak{C}_{P}:=\left\{z \in F^{\prime}: t r_{F^{\prime} / F}\left(z \cdot O_{P}^{\prime}\right) \subseteq O_{P}\right\}
$$

é chamado módulo complementar sobre $O_{P}$.

Proposição 3.31. Usando a notação da definição anterior, temos que:

(a) $\mathfrak{C}_{P}$ é um $O_{P}^{\prime}$-módulo e $O_{P}^{\prime} \subseteq \mathfrak{C}_{P}$;

(b) se $\left\{z_{1}, \ldots, z_{n}\right\}$ é uma base integral de $O_{P}^{\prime}$ sobre $O_{P}$, então:

$$
\mathcal{e}_{P}=\sum_{i=1}^{n} O_{P} \cdot z_{i}^{*}
$$

onde $\left\{z_{1}^{*}, \ldots, z_{n}^{*}\right\}$ é a base dual de $\left\{z_{1}, \ldots, z_{n}\right\}$;

(c) existe um elemento $t \in F^{\prime}$ (dependendo de $P$ ) tal que $\mathcal{C}_{P}=t \cdot O_{P}^{\prime}$; além disso, $v_{P^{\prime}}(t) \leq 0$ para todo $P^{\prime} \mid P$, e, se $t^{\prime} \in F^{\prime}$, então

$$
\mathcal{C}_{P}=t^{\prime} \cdot O_{P}^{\prime} \Longleftrightarrow v_{P^{\prime}}\left(t^{\prime}\right)=v_{P^{\prime}}(t), \forall P^{\prime} \mid P
$$

(d) $\mathcal{C}_{P}=O_{P}^{\prime}$ para quase todo $P \in \mathbb{P}(F)$.

Demonstração. (a) É evidente que $\mathfrak{C}_{P}$ é um $O_{P}^{\prime}$-módulo, e, como o traço de um elemento de $O_{P}^{\prime}$ está em $O_{P}$ (corolário 3.24), temos que $O_{P}^{\prime} \subseteq \mathfrak{C}_{P}$.

(b) Seja $z \in \mathcal{C}_{P}$. Como $\left\{z_{1}^{*}, \ldots, z_{n}^{*}\right\}$ é uma base de $F^{\prime} / F$, temos que existem $x_{1}, \ldots, x_{n} \in$ $F, \mathrm{com}$

$$
z=\sum_{i=1}^{n} x_{i} z_{i}^{*}
$$


Como $z \in \mathcal{C}_{P}$ e $z_{1}, \ldots, z_{n} \in O_{P}^{\prime}$, temos que $t_{F^{\prime} / F}\left(z z_{j}\right) \in O_{P}$ para $1 \leq j \leq n$. Assim,

$$
\begin{aligned}
\operatorname{tr}_{F^{\prime} / F}\left(z z_{j}\right) & =t r_{F^{\prime} / F}\left(\sum_{i=1}^{n} x_{i} z_{i}^{*} z_{j}\right) \\
& =\sum_{i=1}^{n} x_{i} \operatorname{tr} r_{F^{\prime} / F}\left(z_{i}^{*} z_{j}\right) \\
& =x_{j} .
\end{aligned}
$$

Logo, $x_{j} \in O_{P}$ e $z \in \sum_{i=1}^{n} O_{P} \cdot z_{i}^{*}$, ou seja,

$$
\mathfrak{C}_{P} \subseteq \sum_{i=1}^{n} O_{P} \cdot z_{i}^{*}
$$

Reciprocamente, seja

$$
z \in \sum_{i=1}^{n} O_{P} \cdot z_{i}^{*}
$$

Mostraremos que, se $u \in O_{P}^{\prime}$, então $t r_{F^{\prime} / F}(z u) \in O_{P}$, concluindo assim que $z \in \mathcal{C}_{P}$. Sejam

$$
z=\sum_{i=1}^{n} x_{i} z_{i}^{*} \quad \text { e } \quad u=\sum_{i=1}^{n} y_{i} z_{i}
$$

$\operatorname{com} x_{i}, y_{i} \in O_{P}$. Então:

$$
\begin{aligned}
t r_{F^{\prime} / F}(z u) & =t r_{F^{\prime} / F}\left(\sum_{i, j=1}^{n} x_{i} y_{j} z_{i}^{*} z_{j}\right) \\
& =\sum_{i, j=1}^{n} x_{i} y_{j} \operatorname{tr}_{F^{\prime} / F}\left(z_{i}^{*} z_{j}\right) \\
& =\sum_{i=1}^{n} x_{i} y_{i} \in O_{P}
\end{aligned}
$$

Portanto, $z \in \mathcal{C}_{P}$.

(c) Pelo item (b), sabemos que existe uma base $\left\{u_{1}, \ldots, u_{n}\right\}$ de $F^{\prime} / F$ tal que $\mathcal{C}_{P}=$ $\sum_{i=1}^{n} O_{P} \cdot u_{i}$. Seja $0 \neq x \in F$ tal que $v_{P}(x) \geq 0$ e $v_{P}(x) \geq-v_{P^{\prime}}\left(u_{i}\right)$, quaisquer que sejam $P^{\prime} \mid P$ e $i=1, \ldots, n$ (isto é possível pela sobrejetividade de $v_{P}$ ). Então,

$$
v_{P^{\prime}}\left(x u_{i}\right)=e\left(P^{\prime} \mid P\right) \cdot v_{P}(x)+v_{P^{\prime}}\left(u_{i}\right) \geq 0, \forall P^{\prime} \mid P \text { e } i=1, \ldots, n .
$$

Logo, $x u_{i} \in O_{P}^{\prime}$, e, como

$$
\mathcal{C}_{P}=\sum_{i=1}^{n} O_{P} u_{i}
$$


temos:

$$
x \mathfrak{e}_{P}=\sum_{i=1}^{n} O_{P} x u_{i} \Longrightarrow x \mathfrak{e}_{P} \subseteq O_{P}^{\prime} .
$$

Agora, como $x \mathcal{C}_{P}$ é um ideal de $O_{P}^{\prime}$, e $O_{P}^{\prime}$ é um domínio de ideais principais (proposição 3.22), temos que $x \mathrm{e}_{p}=y O_{P}^{\prime}$ para algum $y \in O_{P}^{\prime}$. Fazendo $t=x^{-1} y$, temos $\mathrm{e}_{P}=t \cdot O_{P}^{\prime}$. Como $O_{P}^{\prime} \subseteq \mathcal{e}_{P}$, temos que

$$
\begin{aligned}
1 \in \mathcal{C}_{P} \Longrightarrow t^{-1} \in O_{P}^{\prime} & \Longrightarrow v_{P^{\prime}}\left(t^{-1}\right) \geq 0 \quad, \quad \forall P^{\prime} \mid P \\
& \Longrightarrow v_{P^{\prime}}(t) \leq 0, \forall P^{\prime} \mid P .
\end{aligned}
$$

Finalmente, temos:

$$
\begin{aligned}
t \mathcal{O}_{P}^{\prime}=t^{\prime} O_{P}^{\prime} & \Longleftrightarrow t\left(t^{\prime}\right)^{-1} \in O_{P}^{\prime} \text { e } t^{-1} t^{\prime} \in O_{P}^{\prime} \\
& \Longleftrightarrow v_{P^{\prime}}\left(t\left(t^{\prime}\right)^{-1}\right) \geq 0 \text { e } v_{P^{\prime}}\left(t^{-1} t^{\prime}\right) \geq 0, \forall P^{\prime} \mid P \\
& \Longleftrightarrow v_{P^{\prime}}(t)=v_{P^{\prime}}\left(t^{\prime}\right), \forall P^{\prime} \mid P .
\end{aligned}
$$

(d) Escolhemos $\left\{z_{1}, \ldots, z_{n}\right\}$ uma base de $F^{\prime} / F$. Pelo teorema $3.28,\left\{z_{1}, \ldots, z_{n}\right\}$ e $\left\{z_{1}^{*}, \ldots, z_{n}^{*}\right\}$ são bases integrais para quase todos os lugares $P \in \mathbb{P}(F)$. Logo, pelo item (b), temos $\mathcal{C}_{P}=O_{P}^{\prime}$ para quase todos os lugares $P \in \mathbb{P}(F)$.

Definição 3.32. Considere um lugar $P \in \mathbb{P}(F)$ e o fecho inteiro $O_{P}^{\prime}$ de $O_{P}$ em $F^{\prime}$. Seja $\mathcal{C}_{P}=t \cdot O_{P}^{\prime}$ o módulo complementar sobre $O_{P}$. Então, para cada $P^{\prime} \mid P$, definimos o expoente da diferente de $P^{\prime}$ sobre $P$ por

$$
d\left(P^{\prime} \mid P\right):=-v_{P^{\prime}}(t) \text {. }
$$

Pelo item (c) da proposição anterior, $d\left(P^{\prime} \mid P\right)$ está bem definido, e $d\left(P^{\prime} \mid P\right) \geq 0$. Pelo item (d) da mesma proposição, temos que $d\left(P^{\prime} \mid P\right)=0$ para quase todo $P \in \mathbb{P}(F) e$ $P^{\prime} \mid P$, pois $\mathcal{C}_{P}=O_{P}^{\prime} \Longrightarrow v_{P^{\prime}}(t)=0, \forall P^{\prime} \mid P$. Logo, podemos definir o divisor

$$
\operatorname{Diff}\left(F^{\prime} / F\right):=\sum_{P \in \mathbb{P}(F)} \sum_{P^{\prime} \mid P} d\left(P^{\prime} \mid P\right) \cdot P^{\prime} \text {. }
$$

Este divisor está em $D_{F^{\prime}}$, e é chamado de diferente de $F^{\prime} / F$. Observamos que:

$$
\operatorname{Diff}\left(F^{\prime} / F\right) \geq 0 \text {. }
$$

Observação 3.33. Da definição acima, segue imediatamente uma forma alternativa de caracterização de $\mathcal{C}_{P}$ : para $z \in F^{\prime}$, temos:

$$
z \in \mathfrak{C}_{P} \Longleftrightarrow v_{P^{\prime}}(z) \geq-d\left(P^{\prime} \mid P\right), \forall P^{\prime} \mid P .
$$


De fato,

$$
\begin{aligned}
z \in \mathcal{C}_{P} & \Longrightarrow z=t \cdot x, x \in O_{P^{\prime}} \\
& \Longrightarrow v_{P^{\prime}}(z)=v_{P^{\prime}}(t)+v_{P^{\prime}}(x) \geq v_{P^{\prime}}(t)=-d\left(P^{\prime} \mid P\right), \forall P^{\prime} \mid P .
\end{aligned}
$$

Reciprocamente,

$$
v_{P^{\prime}}(z) \geq-d\left(P^{\prime} \mid P\right), \forall P^{\prime}\left|P \Longrightarrow v_{P^{\prime}}(z)-v_{P^{\prime}}(t) \geq 0, P^{\prime}\right| P,
$$

onde $t \in F^{\prime} \quad$ e $\mathcal{C}_{P}=t \cdot O_{P}^{\prime}$. Logo,

$$
v_{P^{\prime}}\left(\frac{z}{t}\right) \geq 0, \forall P^{\prime} \mid P
$$

e, portanto,

$$
\frac{z}{t}=u \in O_{P}^{\prime} \Longrightarrow z=t \cdot u \Longrightarrow z \in \mathcal{C}_{P}
$$

Lembraremos alguns conceitos que serão úteis no que segue.

- Seja $\mathcal{A}_{F}$ o espaço dos adeles de $F / K$. Para um divisor $A \in D_{F}$, temos que:

$$
\mathcal{A}_{F}(A)=\left\{\alpha \in \mathcal{A}_{F} \quad: \quad v_{P}(\alpha) \geq-v_{P}(A), \forall P \in \mathbb{P}\left(F^{\prime}\right)\right\}
$$

é um $K$-subespaço de $\mathcal{A}_{F}$.

- Definindo adele principal, temos que $F$ é naturalmente imerso em $\mathcal{A}_{F}$.

- Uma diferencial de Weil $\omega$ em $F / K$ é uma função $K$-linear $\omega: \mathcal{A}_{F} \longrightarrow K$ que se anula em $\mathcal{A}_{F}(A)+F$ para algum divisor $A \in D_{F}$.

- Se $\omega \neq 0$ é uma diferencial de Weil de $F / K$, então o seu divisor $(\omega) \in D_{F}$ é por definição

$$
(\omega)=\max \left\{A \in D_{F}: \omega \text { se anula em } \mathcal{A}_{F}(A)+F\right\}
$$

Definição 3.34. Seja

$$
\mathcal{A}_{F^{\prime} / F}:=\left\{\alpha \in \mathcal{A}_{F^{\prime}}: \alpha_{P^{\prime}}=\alpha_{Q^{\prime}} \text { sempre que } P^{\prime} \cap F=Q^{\prime} \cap F\right\} .
$$

A função traço $\operatorname{tr}_{F^{\prime} / F}: F^{\prime} \longrightarrow F$ pode ser estendida a uma função F-linear (também denotada por $\operatorname{tr}_{F^{\prime} / F}$ de $\mathcal{A}_{F^{\prime} / F}$ para $\mathcal{A}_{F}$ fazendo

$$
\operatorname{tr}_{F^{\prime} / F}:\left\{\begin{array}{rl}
\mathcal{A}_{F^{\prime} / F} & \longrightarrow \mathcal{A}_{F} \\
\alpha & \longmapsto r_{F^{\prime} / F}(\alpha)
\end{array},\right.
$$

onde $\left(\operatorname{tr}_{F^{\prime} / P}(\alpha)\right)_{P}:=\operatorname{tr}_{F^{\prime} / F}\left(\alpha_{P^{\prime}}\right)$, com $P^{\prime} \mid P$.

Observe que $\alpha_{P^{\prime}}$ é o mesmo elemento de $F^{\prime}$, qualquer que seja $P^{\prime} \mid P$, pois $\alpha \in \mathcal{A}_{F^{\prime} / F^{\prime}}$. Temos ainda que $\alpha_{P^{\prime}} \in O_{P^{\prime}}$ para quase todo $P^{\prime} \in \mathbb{P}\left(F^{\prime}\right)$, e, conseqüentemente, pelo 
corolário 3.24 , temos que $t r_{F^{\prime} / F}\left(\alpha_{P^{\prime}}\right) \in O_{P}$ para quase todo $P \in \mathbb{P}(F)$, caracterizando efetivamente um adele. É fácil ver também que o traço de um adele principal de $\mathcal{A}_{F^{\prime} / F}$ é um adele principal de $\mathcal{A}_{F}$ consistente ainda com a função traço ordinária $\operatorname{tr}_{F^{\prime} / F}: F^{\prime} \longrightarrow F$.

A demonstração do próximo teorema pode ser encontrada em [11] (página 83).

Teorema 3.35. Na situação acima, para cada diferencial de Weil $\omega$ de $F / K$, existe uma única diferencial de Weil $\omega^{\prime}$ de $F^{\prime} / K^{\prime}$, chamada de cotraço de $\omega$ em $F^{\prime} / F$, e denotada por $\operatorname{Cotr}_{F^{\prime} / F}(\omega)$, tal que

$$
t r_{K^{\prime} / K}\left(\omega^{\prime}(\alpha)\right)=\omega\left(\operatorname{tr}_{F^{\prime} / F}(\alpha)\right), \forall \alpha \in \mathcal{A}_{F^{\prime} / F}
$$

Se $0 \neq(\omega) \in D_{F}$ é o divisor de $\omega$, então

$$
\left(\operatorname{Cotr}_{F^{\prime} / F}(\omega)\right)=\operatorname{Con}_{F^{\prime} / F}((\omega))+\operatorname{Diff}\left(F^{\prime} / F\right)
$$

Enunciaremos agora um dos resultados mais importantes do nosso trabalho.

Teorema 3.36 (Fórmula do gênero de Hurwitz). Seja $F / K$ um corpo de funções algébricas de gênero $g$ e $F^{\prime} / F$ uma extensão finita e separável. Seja $K^{\prime}$ o corpo das constantes de $F^{\prime}$ e g' o gênero de $F^{\prime} / K^{\prime}$. Entâo:

$$
2 g^{\prime}-2=\frac{\left[F^{\prime}: F\right]}{\left[K^{\prime}: K\right]} \cdot(2 g-2)+\operatorname{deg}\left(\operatorname{Diff}\left(F^{\prime} / F\right)\right) \text {. }
$$

Demonstração. Considere uma diferencial de Weil $\omega \neq 0$ em $F / K$. Pelo teorema anterior, temos que:

$$
\left(\operatorname{Cotr}_{F^{\prime} / F}(\omega)\right)=\operatorname{Con}_{F^{\prime} / F}((\omega))+\operatorname{Diff}\left(F^{\prime} / F\right)
$$

o que implica

$$
\operatorname{deg}\left(\left(\operatorname{Cotr}_{F^{\prime} / F}(\omega)\right)\right)=\operatorname{deg}\left(\operatorname{Con}_{F^{\prime} / F}((\omega))\right)+\operatorname{deg}\left(\operatorname{Diff}\left(F^{\prime} / F\right)\right) .
$$

Mas, lembremos (corolário 2.45) que o grau de um divisor canônico em $F / K$ é $2 g-2$ $\left(2 g^{\prime}-2\right.$ em $\left.F^{\prime} / K^{\prime}\right)$, e ainda sabemos, pelo corolário 3.13 , que

$$
\operatorname{deg}\left(\operatorname{Con}_{F^{\prime} / F}((\omega))\right)=\frac{\left[F^{\prime}: F\right]}{\left[K^{\prime}: K\right]} \cdot \operatorname{deg}((\omega))
$$

Então:

$$
2 g^{\prime}-2=\frac{\left[F^{\prime}: F\right]}{\left[K^{\prime}: K\right]} \cdot(2 g-2)+\operatorname{deg}\left(\operatorname{Diff}\left(F^{\prime} / F\right)\right)
$$




\subsection{A Diferente}

Nesta seção, assumiremos $F^{\prime} / F$ finita e separável. Para $P \in \mathbb{P}(F)$ e $P^{\prime} \in \mathbb{P}\left(F^{\prime}\right)$, com $P^{\prime} \mid P$, já definimos em seções anteriores o índice de ramificação $e\left(P^{\prime} \mid P\right)$ e o expoente da diferente $d\left(P^{\prime} \mid P\right)$. O resultado central aqui apresentado é o Teorema da Diferente de Dedekind, que mostra a existência de uma estreita relação entre estes dois números. Nesta seção, também estabelecemos a nomenclatura relacionada à ramificação de lugares, e finalizaremos com a apresentação de dois resultados que serão utilizados em seções posteriores.

Teorema 3.37 (Teorema da diferente de Dedekind). Usando a notaçấo acima, para qualquer que seja $P^{\prime} \mid P$, temos:

(a) $d\left(P^{\prime} \mid P\right) \geq e\left(P^{\prime} \mid P\right)-1$;

(b) $d\left(P^{\prime} \mid P\right)=e\left(P^{\prime} \mid P\right)-1 \Longleftrightarrow e\left(P^{\prime} \mid P\right)$ nâo é divisível pela característica de $K$.

Em particular, se car $(K)=0$, então $d\left(P^{\prime} \mid P\right)=e\left(P^{\prime} \mid P\right)-1$.

Observação 3.38. Pelo item (b), observamos que, se $P^{\prime} \mid P$ é não-ramificado, isto é, se $e\left(P^{\prime} \mid P\right)=1$, então $P^{\prime}$ não está no suporte de Diff $\left(F^{\prime} \mid F\right)$. Isto serve como auxilio para o cálculo do gênero de $F^{\prime}$, pela fórmula de Hurwitz.

Enunciaremos dois lemas que nos permitirão provar cada um dos itens do teorema acima.

Lema 3.39. ([11], página 89) Seja $F^{*} / F$ uma extensão de corpos de funções, $P \in \mathbb{P}(F)$ e $P^{*} \in \mathbb{P}\left(F^{*}\right)$, com $P^{*} \mid P$. Considere um automorfismo $\sigma$ de $F^{*} / F$. Então

$$
\sigma\left(P^{*}\right):=\left\{\sigma(z): z \in P^{*}\right\}
$$

é um lugar de $F^{*}$, e temos:

(a) $v_{\sigma\left(P^{*}\right)}(y)=v_{P^{*}}\left(\sigma^{-1}(y)\right), \forall y \in F^{*} ;$

(b) $\sigma\left(P^{*}\right) \mid P$;

(c) $e\left(\sigma\left(P^{*}\right) \mid P\right)=e\left(P^{*} \mid P\right)$ e $f\left(\sigma\left(P^{*}\right) \mid P\right)=f\left(P^{*} \mid P\right)$.

Demonstração do teorema 3.37(a). Seja $O_{P}^{\prime}$ o fecho inteiro de $O_{P}$ em $F^{\prime}$, e $\mathcal{C}_{P}$ o módulo complementar sobre $O_{P}$. Pela observação 3.33 , temos que

$$
z \in \mathcal{C}_{P} \Longleftrightarrow-v_{P^{\prime}}(z) \leq d\left(P^{\prime} \mid P\right), \forall P^{\prime} \mid P .
$$

Assim, a idéia é mostrar que, se $t \in F^{\prime}$ e $v_{P^{\prime}}(t)=1-e\left(P^{\prime} \mid P\right)$, qualquer que seja $P^{\prime} \mid P$, então $t \in \mathcal{C}_{P}$, ou seja, mostraremos que $\operatorname{tr}_{F^{\prime} / F}\left(t \cdot O_{P}^{\prime}\right) \subseteq O_{P}$. 
Considere uma extensão de Galois finita $F^{*} / F$ tal que $F \subseteq F^{\prime} \subseteq F^{*}$. Como $F^{\prime} / F$ é finita e separável, e sendo $\left[F^{\prime}: F\right]=n$, podemos tomar $n$ automorfismos $\sigma_{1}, \ldots, \sigma_{n}$ de $F^{*} / F$, cujas restrições a $F^{\prime}$ sejam duas a duas distintas. Assim, para $z \in O_{P}^{\prime}$ e $t \in F^{\prime}$, com $v_{P^{\prime}}(t)=1-e\left(P^{\prime} \mid P\right)$, qualquer que seja $P^{\prime} \mid P$ (tal $t$ existe, pelo teorema 2.22 , da aproximação fraca), temos, pelas propriedades da função traço:

$$
t r_{F^{\prime} / F}(t z)=\sum_{i=1}^{n} \sigma_{i}(t z) \quad(\diamond)
$$

Agora, fixamos $P^{*}$ em $F^{*}$ tal que $P^{*} \mid P$, e a partir dos automorfismos, conforme o lema 3.39 , obtemos outros lugares de $F^{*}$, dados por $P_{i}^{*}:=\sigma_{i}^{-1}\left(P^{*}\right)$, e, a partir destes, obtemos os lugares de $F^{\prime}$ dados por $P_{i}^{\prime}=P_{i}^{*} \cap F^{\prime}$ (observamos que $P_{i}^{*} \mid P$ e $P_{i}^{\prime} \mid P$ ). Como $z \in O_{P}^{\prime}$, temos que $\sigma_{i}(z) \in O_{P}^{\prime}$, pois

$$
\sum_{j=0}^{n} a_{j} z^{j}=0 \Longrightarrow \sum_{j=0}^{n} a_{j} \sigma_{i}(z)^{j}=0,
$$

onde $a_{j} \in O_{P}$. Logo, $v_{P^{*}}\left(\sigma_{i}(z)\right) \geq 0$. Assim, obtemos

$$
\begin{aligned}
v_{P^{*}}\left(\sigma_{i}(t z)\right) & =v_{P^{*}}\left(\sigma_{i}(t)\right)+v_{P^{*}}\left(\sigma_{i}(z)\right) \\
& \geq v_{P^{*}}\left(\sigma_{i}(t)\right)=v_{P_{i}^{*}}(t) \quad \text { (lema 3.39) } \\
& =e\left(P_{i}^{*} \mid P_{i}^{\prime}\right) \cdot v_{P_{i}^{\prime}}(t) \\
& =e\left(P_{i}^{*} \mid P_{i}^{\prime}\right) \cdot\left(1-e\left(P_{i}^{\prime} \mid P\right)\right) \\
& >-e\left(P_{i}^{*} \mid P_{i}^{\prime}\right) \cdot e\left(P_{i}^{\prime} \mid P\right) \\
& =-e\left(P_{i}^{*} \mid P\right) \\
& =-e\left(P^{*} \mid P\right) \quad(\text { lema 3.39) }
\end{aligned}
$$

Então, usando ( $\diamond)$, temos:

$$
\begin{aligned}
& v_{P^{*}}\left(t r_{F^{\prime} / F^{*}}(t z)\right)=v_{P^{*}}\left(\sum \sigma_{i}(t z)\right)>-e\left(P^{*} \mid P\right) \\
& \Longrightarrow e\left(P^{*} \mid P\right) \cdot v_{P}\left(t r_{F^{\prime} / F}(t z)\right)>-e\left(P^{*} \mid P\right) \\
& \Longrightarrow v_{P}\left(t_{F^{\prime} / F}(t z)\right) \geq 0 .
\end{aligned}
$$

Como $z \in O_{P}^{\prime}$ foi escolhido arbitrariamente, temos $\operatorname{tr}_{F^{\prime} / F}\left(t \cdot O_{P}^{\prime}\right) \subseteq O_{P}$, isto é, $t \in \mathcal{C}_{P}$.

O próximo lema é essencial para a demonstração da parte (b) do teorema 3.37 .

Lema 3.40. ([11], página 91) Seja $P \in \mathbb{P}(F)$ e $P_{1}, \ldots, P_{r} \in \mathbb{P}\left(F^{\prime}\right)$ todas as extensões de $P$ em $F^{\prime} / F$. Considere o corpo residual $M:=O_{P} / P$ e $M_{i}:=O_{P_{i}} / P_{i} \supseteq M$, e as correspondentes funções de classe de resíduo $\pi: O_{P} \longrightarrow M$ e $\pi_{i}: O_{P_{i}} \longrightarrow M_{i}$ (para $i=1, \ldots, r)$. Então, para todo $u \in O_{P}^{\prime}$ (o fecho inteiro de $O_{P}$ em $F^{\prime}$ ), temos:

$$
\pi\left(t r_{F^{\prime} / F}(u)\right)=\sum_{i=1}^{r} e\left(P_{i} \mid P\right) \cdot \operatorname{tr}_{M_{i} / M}\left(\pi_{i}(u)\right) .
$$


Demonstração do teorema 3.5.1(b). Usaremos a notação do lema 3.40 acima, e abreviaremos $e_{i}=e\left(P_{i} \mid P\right)$. Seja $P^{\prime}=P_{1}$ e $e:=e\left(P^{\prime} \mid P\right)$. Devemos mostrar que:

$$
d\left(P^{\prime} \mid P\right)=e-1 \Longleftrightarrow \operatorname{car}(K) \text { não divide } e .
$$

Primeiramente, assumiremos que $\operatorname{car}(K)$ não divide $e$. Suponhamos $d\left(P^{\prime} \mid P\right) \geq e$, e tomemos $t \in F^{\prime}$ tal que $\mathcal{C}_{P}=t \cdot O_{P}^{\prime}$. Logo:

$$
v_{P^{\prime}}(t) \leq-e \quad \text { e } \quad \operatorname{tr}_{F^{\prime} / F}\left(t \cdot O_{P}^{\prime}\right) \subseteq O_{P}
$$

Como $K$ é perfeito, temos que a extensão $M_{1} / M$ é separável, e, portanto, $\operatorname{tr}_{M_{1} / M} \not \equiv 0$. Logo, podemos tomar $y_{0} \in O_{P}^{\prime}$ tal que $\operatorname{tr}_{M_{1} / M}\left(\pi_{1}\left(y_{0}\right)\right) \neq 0$. Pelo teorema 2.22 (da aproximação fraca), existe $y \in F^{\prime}$ tal que

$$
v_{P^{\prime}}\left(y-y_{0}\right)>0 \quad \text { e } \quad v_{P_{i}}(y) \geq \max \left\{1, e_{i}+v_{P_{i}}(t)\right\}, 2 \leq i \leq r
$$

Podemos observar que $v_{P^{\prime}}(y) \geq 0$, pois

$$
v_{P^{\prime}}(y)=v_{P^{\prime}}\left(y-y_{0}+y_{0}\right) \geq \min \left\{v_{P^{\prime}}\left(y-y_{0}\right), v_{P^{\prime}}\left(y_{0}\right)\right\} .
$$

Assim, temos que $v_{P_{i}}(y) \geq 0$, qualquer que seja $i=1, \ldots, r$, e, portanto, $y \in O_{P}^{\prime}$.

Pelo lema 3.40, temos

$$
\pi\left(\operatorname{tr}_{F^{\prime} / F}(y)\right)=e \cdot \operatorname{tr}_{M_{1} / M}\left(\pi_{1}(y)\right)+\sum_{i=2}^{r} e_{i} \cdot \operatorname{tr}_{M_{i} / M}\left(\pi_{i}(y)\right) .
$$

Agora, observamos os seguintes fatos:

- $v_{P^{\prime}}\left(y-y_{0}\right)>0 \Longrightarrow y\left(P^{\prime}\right)=y_{0}\left(P^{\prime}\right) \Longrightarrow \pi_{1}(y)=\pi_{1}\left(y_{0}\right)$

- $\operatorname{para} 2 \leq i \leq r$, temos

$$
v_{P_{i}}(y) \geq 1 \Longrightarrow \pi_{i}(y)=0 \Longrightarrow \operatorname{tr}_{M_{i} / M}\left(\pi_{i}(y)\right)=0 ;
$$

- como e não é divisível pela característica de $K$, temos $e \neq 0$ em $M$.

Logo:

$$
\pi\left(\operatorname{tr}_{F^{\prime} / F}(y)\right)=e \cdot \operatorname{tr}_{M_{1} / M}\left(\pi\left(y_{0}\right)\right) \neq 0 .
$$

Como $y \in O_{P}^{\prime}$, temos $t r_{F^{\prime} / F}(y) \in O_{P}$. Mas, $\pi\left(t r_{F^{\prime} / F}(y)\right) \neq 0$ em $M$. Logo:

$$
\operatorname{tr}_{F^{\prime} / F}(y) \notin P \Longrightarrow v_{P}\left(\operatorname{tr}_{F^{\prime} / F}(y)\right)=0 \text {. }
$$

Agora, tomamos $x \in F$, com $v_{P}(x)=1$, e temos

$$
t r_{F^{\prime} / F}\left(x^{-1} y\right)=x^{-1} \cdot \operatorname{tr}_{F^{\prime} / F}(y) \notin O_{P} \quad(\star) .
$$

Por outro lado, temos que $x^{-1} y t^{-1} \in O_{P}^{\prime}$, pois

$$
v_{P^{\prime}}\left(x^{-1} y t^{-1}\right)=-e+v_{P^{\prime}}(y)-v_{P^{\prime}}(t)=\underbrace{v_{P^{\prime}}(y)}_{\geq 0}-\underbrace{e-v_{P^{\prime}}(t)}_{\geq 0} \geq 0 .
$$


Logo, $x^{-1} y t^{-1} \in O_{P^{\prime}}$, e ainda

$$
\begin{aligned}
v_{P_{i}}\left(x^{-1} y t^{-1}\right) & =-v_{P_{i}}(x)+v_{P_{i}}(y)-v_{P_{i}}(t) \\
& =-e_{i}-v_{P_{i}}(t)+v_{P_{i}}(y) \\
& =v_{P_{i}}(y)-\left(e_{i}+v_{P_{i}}(t)\right) \geq 0, \forall i=2, \ldots, r .
\end{aligned}
$$

Logo, $x^{-1} y t^{-1} \in O_{P_{i}}$, e, portanto,

$$
x^{-1} y t^{-1} \in O_{P}^{\prime} \Longrightarrow x^{-1} y \in t \cdot O_{P}^{\prime}=\mathcal{C}_{P} \Longrightarrow t r_{F^{\prime} / F}\left(x^{-1} y\right) \in O_{P}
$$

contradizendo $(\star)$.

Para provar a recíproca, assumiremos que a característica de $K$ divide $e$, e provaremos que $d\left(P^{\prime} \mid P\right) \geq e$. Escolhamos $u \in F^{\prime}$ tal que

$$
v_{P^{\prime}}(u)=-e \quad \text { e } \quad v_{P_{i}}(u) \geq-e_{i}+1(i=2, \ldots, r) .
$$

Como antes, tomamos $x \in F$ tal que $v_{P}(x)=1$. Para todo $z \in O_{P}^{\prime}$, temos

$$
v_{P^{\prime}}(x u z)=v_{P^{\prime}}(x)+v_{P^{\prime}}(u)+v_{P^{\prime}}(z)=e-e+v_{P^{\prime}}(z) \geq 0
$$

e

$$
v_{P_{i}}(x u z)=v_{P_{i}}(x)+v_{P_{i}}(u)+v_{P_{i}}(z) \geq e_{i}-e_{i}+1+v_{P_{i}}(z)>0 \quad(i=2, \ldots, r) .
$$

Logo, $x u z \in O_{P}^{\prime}$, e pelo lema 3.40 , temos

$$
\begin{aligned}
\pi\left(t r_{F^{\prime} / F}(x u z)\right) & =e \cdot \operatorname{tr}_{M_{1} / M}\left(\pi_{1}(x u z)\right)+\sum_{i=2}^{r} e_{i} \cdot \operatorname{tr}_{M_{i} / M}\left(\pi_{i}(x u z)\right) \\
& =e \cdot t r_{M_{1} / M}\left(\pi_{1}(x u z)\right)=0
\end{aligned}
$$

pois $\pi_{i}(x u z)=0\left(v_{P_{i}}(x u z)>0\right)$ para todo $i=2, \ldots, r$, e $e=n \cdot \operatorname{car}(K), n \in \mathbb{N}$. Logo,

$$
t r_{F^{\prime} / F}(x u z) \in P \Longrightarrow x \cdot t r_{F^{\prime} / F}(u z) \in P
$$

e, como $v_{P}(x)=1$, temos

$$
v_{P}\left(t r_{F^{\prime} / F}(u z)\right) \geq 0 \Longrightarrow t r_{F^{\prime} / F}(u z) \in O_{P}
$$

Como $z \in O_{P}^{\prime}$ é arbitrário, temos que $u \in \mathcal{C}_{P}$, e, assim,

$$
v_{P^{\prime}}(u) \geq-d\left(P^{\prime} \mid P\right) \Longrightarrow d\left(P^{\prime} \mid P\right) \geq e .
$$

Como $P^{\prime}=P_{1}$ foi escolhido arbitrariamente dentre $P_{1}, \ldots, P_{r}$, temos que o item (b) do teorema está provado.

Definição 3.41. Seja $F^{\prime} / F$ uma extensão de corpos de funções e $P \in \mathbb{P}(F)$.

(a) Dizemos que $P$ é ramificado em $F^{\prime} / F$ se existe pelo menos um lugar $P^{\prime} \in \mathbb{P}\left(F^{\prime}\right)$ sobre $P$ tal que $e\left(P^{\prime} \mid P\right)>1$. 
(b) Dizemos que $P$ é não-ramificado em $F^{\prime} / F$ se e $\left(P^{\prime} \mid P\right)=1$ qualquer que seja $P^{\prime} \mid P, \operatorname{com} P^{\prime} \in \mathbb{P}\left(F^{\prime}\right)$.

(c) $P$ é totalmente ramificado em $F^{\prime} / F$ se existe uma única extensão $P^{\prime} \in \mathbb{P}\left(F^{\prime}\right)$ de $P$ em $F^{\prime}$, e o indice de ramificação é $e\left(P^{\prime} \mid P\right)=\left[F^{\prime}: F\right]$.

Corolário 3.42. Se $F^{\prime} / F$ é uma extensão finita e separável de corpos de funções algébricas, então quase todos os lugares $P \in \mathbb{P}(F)$ são não-ramificados em $F^{\prime} / F$.

Demonstração. Segue da definição 3.32 que $d\left(P^{\prime} \mid P\right)=0$ para quase todo $P \in \mathbb{P}(F)$. Logo, pelo teorema 3.37 ,

$$
0 \geq e\left(P^{\prime} \mid P\right)-1 \Longrightarrow e\left(P^{\prime} \mid P\right)=1,
$$

para quase todo $P \in \mathbb{P}(F)$.

Os dois próximos resultados nos servirão de suporte em demonstrações futuras.

Teorema 3.43. ([11], página 96) Suponha $F^{\prime}=F(y)$ uma extensão finita e separável de $F, e\left[F^{\prime}: F\right]=n$. Seja $P \in \mathbb{P}(F)$ tal que o polinômio minimal $\varphi(T)$ de y sobre $F$ tenha coeficientes em $O_{P}$ (isto é, y é inteiro sobre $O_{P}$ ), e sejam $P_{1}, \ldots, P_{r} \in \mathbb{P}\left(F^{\prime}\right)$ todos os lugares de $F^{\prime}$ que estão sobre $P$. Então, temos:

(a) $d\left(P_{i} \mid P\right) \leq v_{P_{i}}\left(\varphi^{\prime}(y)\right)$ para $1 \leq i \leq r$;

(b) $\left\{1, y, y^{2}, \ldots, y^{n-1}\right\}$ é uma base integral de $F^{\prime}$ em $P$ se, e somente se, $d\left(P_{i} \mid P\right)=$ $v_{P_{i}}\left(\varphi^{\prime}(y)\right)$, com $i=1, \ldots, r$.

$\left(\varphi^{\prime}(T)\right.$ denota a derivada de $\varphi(T)$ em $\left.F[T]\right)$.

Proposição 3.44. ([11], página 100) Seja $F^{\prime} / F$ uma extensão finita e separável de corpos de funções, $P \in \mathbb{P}(F)$ e $P^{\prime} \in \mathbb{P}\left(F^{\prime}\right)$ com $P^{\prime} \mid P$. Suponha que $P$ é totalmente ramificado, isto é, $e\left(P^{\prime} \mid P\right)=\left[F^{\prime}: F\right]=: n$. Seja $t \in F^{\prime}$ um elemento primo de $P^{\prime}$, e considere o polinômio mimimal $\varphi(T) \in F[T]$ de $t$ sobre $F$. Então, $d\left(P^{\prime} \mid P\right)=v_{P^{\prime}}\left(\varphi^{\prime}(t)\right)$ e $\left\{1, t, \ldots, t^{n-1}\right\}$ é uma base integral de $F^{\prime} / F$ em $P$.

\subsection{Extensões do Corpo das Constantes}

Esta seção tem como principais resultados o item (d) da proposição 3.45 (a seguir) e um caso especial que ocorre na proposição 3.47. Ambos serão utilizados na seção seguinte.

Consideramos um corpo de funções algébricas $F / K$, com corpo das constantes $K$, onde $K$ é perfeito. Lembramos que $\Phi \supseteq F$ denota um corpo fixo algebricamente fechado.

Lembramos ainda que, se $K^{\prime} \supseteq K$ é uma extensão algébrica (com $K^{\prime} \subseteq \Phi$ ), então o compósito $F^{\prime}=K^{\prime} F$ é um corpo de funções sobre $K^{\prime}$, e, portanto, seu corpo de constantes é uma extensão finita de $K^{\prime}$. A próxima proposição mostra, entre oütras coisas, que neste caso $K^{\prime}$ é o próprio corpo das constantes. 
Proposição 3.45. Seja $F^{\prime}=F K^{\prime}$ uma extensão algébrica constante de $F / K$. Então:

(a) $K^{\prime}$ é o corpo das constantes de $F^{\prime}$;

(b) todo subconjunto de $F$ linearmente independente sobre $K$, mantém-se linearmente independente sobre $K^{\prime}$;

(c) $[F: K(x)]=\left[F^{\prime}: K^{\prime}(x)\right], \forall x \in F \backslash K$;

(d) todos os lugares $P \in \mathbb{P}(F)$ são não-ramificados em $F^{\prime} / F$.

Para demonstrar tal proposição, usaremos o seguinte lema:

Lema 3.46. ([11], página 102) Suponha que $\alpha \in \Phi$ é algébrico sobre $K$. Então, $[K(\alpha)$ : $K]=[F(\alpha): F]$.

Demonstração da proposição 3.45. (a) Seja $\gamma \in F^{\prime}$ algébrico sobre $K^{\prime}$. Então, $\gamma$ é algébrico sobre $K$, e, como $F^{\prime}=K^{\prime} F$, temos que

$$
\gamma=\frac{\sum_{i=1}^{m} \alpha_{i} \beta_{i}}{\sum_{j=m+1}^{r} \alpha_{j} \beta_{j}}
$$

com $\alpha_{i}, \alpha_{j} \in K^{\prime}$ e $\beta_{i}, \beta_{j} \in F$, ou seja, existem $\alpha_{1}, \ldots, \alpha_{r} \in K^{\prime}$ tais que $\gamma \in$ $F\left(\alpha_{1}, \ldots, \alpha_{r}\right)$.

Como $K$ é perfeito, e $K^{\prime}$ é extensão algébrica de $K$, temos que a extensão $K\left(\alpha_{1}, \ldots, \alpha_{r}\right) / K$ é finita e separável, e, portanto, $K\left(\alpha_{1}, \ldots, \alpha_{r}\right)=K(\alpha)$ para algum $\alpha \in K^{\prime}$. Sendo $\gamma$ algébrico sobre $K^{\prime}$, e, conseqüentemente, sobre $K$, temos que $K(\alpha, \gamma) / K$ é finita e separável. Logo, existe $\beta \in F^{\prime}$ tal que $K(\alpha, \gamma)=K(\beta)$. Logo, $F(\alpha, \gamma)=F(\beta)$. Mas, $\gamma \in F\left(\alpha_{1}, \ldots, \alpha_{r}\right)=F(\alpha)$; logo, $F(\alpha)=F(\beta)$. Assim, pelo lema 3.46, temos

$$
[K(\beta): K]=[F(\beta): F]=[F(\alpha): F]=[K(\alpha): K] .
$$

Logo, como $K(\alpha) \subseteq K(\beta)$, temos

$$
K(\alpha)=K(\beta) \Longrightarrow \gamma \in K(\alpha) \subseteq K^{\prime}
$$

Portanto, $\tilde{K}^{\prime}=K^{\prime}$.

(b) Sejam $y_{1}, \ldots, y_{r} \in F$ linearmente independentes sobre $K$, e suponha que

$$
\sum_{i=1}^{r} \gamma_{i} y_{i}=0, \operatorname{com} \gamma_{i} \in K^{\prime} \quad(\mathbf{I})
$$

Provaremos que $\gamma_{i}=0$ para $i=1, \ldots, r$. 
Temos que $K\left(\gamma_{1}, \ldots, \gamma_{r}\right) / K$ é finita e separável. Logo, existe $\alpha \in K^{\prime}$ tal que $\gamma_{i} \in$ $K(\alpha), i=1, \ldots, r$. Sendo $[K(\alpha): K]=: n$, temos que $1, \alpha, \ldots, \alpha^{n-1}$ é base de $K(\alpha) / K$, e, portanto,

$$
\gamma_{i}=\sum_{j=0}^{n-1} c_{i j} \cdot \alpha^{j} \quad, \quad \operatorname{com} c_{i j} \in K
$$

De $(\mathbf{I})$, obtemos

$$
0=\sum_{i=1}^{r}\left(\sum_{j=0}^{n-1} c_{i j} \cdot \alpha^{j}\right) y_{i}=\sum_{j=0}^{n-1}\left(\sum_{i=1}^{r} c_{i j} \cdot y_{i}\right) \alpha^{j} \quad(\mathbf{I I}),
$$

$\operatorname{com} \sum c_{i j} \cdot y_{i} \in F$.

Como $[F(\alpha): F]=[K(\alpha): K]=n$ (lema 3.46$)$, temos que $1, \alpha, \ldots, \alpha^{n-1}$ são linearmente independentes sobre $F$, e, por (II), temos

$$
\sum_{i=1}^{r} c_{i j} \cdot y_{i}=0 \text { para } j=0,1, \ldots, n-1 .
$$

Mas, como $y_{1}, \ldots, y_{r}$ são linearmente independentes sobre $K$, temos que $c_{i j}=0$, e, conseqüentemente, $\gamma_{i}=0$ para $i=1, \ldots, r$.

(c) Como $F^{\prime}=F K^{\prime}$, é relativamente simples mostrar que $\left[F^{\prime}: K^{\prime}(x)\right] \leq[F: K(x)]$. Para mostrar que $\left[F^{\prime}: K^{\prime}(x)\right] \geq[F: K(x)]$, provaremos que, se $z_{1}, \ldots, z_{s} \in F$ são linearmente independentes sobre $K(x)$, então estes também são linearmente independentes sobre $K^{\prime}(x)$.

Suponhamos que não, ou seja,

$$
\sum_{i=1}^{s} f_{i}(x) z_{i}=0 \quad \text { (III) }
$$

onde $f_{i}(x) \in K^{\prime}(x)$ são não todos nulos. Podemos assumir que $f_{i}(x) \in K^{\prime}[x]$ (basta multiplicar pelo MMC dos denominadores). Temos que (III) afirma que existe uma dependência linear dos elementos do conjunto $\left\{x^{j} z_{i}: 1 \leq i \leq s\right.$ e $\left.j \geq 0\right\}$ sobre $K^{\prime}$. Como $x^{j} z_{i} \in F$, temos um subconjunto de $F$ linearmente dependente sobre $K^{\prime}$. Assim, pelo item (b), temos que os elementos $x^{j} z_{i} \in F$ são linearmente dependentes sobre $K$. Logo, $z_{1}, z_{2}, \ldots, z_{s}$ são linearmente dependentes sobre $K(x)$, uma contradição.

(d) Suponhamos primeiramente que $F^{\prime} / F$ seja finita. Logo, $\left[K^{\prime}: K\right]<\infty$; sendo $K$ perfeito, temos então que $K^{\prime}=K(\alpha)$, e, como $F^{\prime}=F K^{\prime}$, temos $F^{\prime}=F(\alpha)$, e que o polinômio minimal $\varphi(T)$ de $\alpha$ sobre $K$ continua irredutível sobre $F$ (lema 3.46). Seja $P \in \mathbb{P}(F)$ e $P^{\prime} \in \mathbb{P}\left(F^{\prime}\right)$ com $P^{\prime} \mid P$. Como $K \subseteq O_{P}$, segue, do teorema 3.43, que o expoente da diferente $d\left(P^{\prime} \mid P\right)$ satisfaz

$$
0 \leq d\left(P^{\prime} \mid P\right) \leq v_{P^{\prime}}\left(\varphi^{\prime}(\alpha)\right)
$$


Como $\alpha$ é separável sobre $K$, temos $\varphi^{\prime}(\alpha) \neq 0$, e como $\varphi^{\prime}(\alpha) \in K^{\prime}$, temos $v_{P^{\prime}}\left(\varphi^{\prime}(\alpha)\right)=$ 0 . Logo:

$$
d\left(P^{\prime} \mid P\right)=0 \Longrightarrow e\left(P^{\prime} \mid P\right)=1 \text {. }
$$

Caso a extensão $F^{\prime} / F$ não seja finita, tomemos $t \in F^{\prime}$ um elemento primo de $P^{\prime}$, isto é, $v_{P^{\prime}}(t)=1$. Como $F^{\prime}=F K^{\prime}$, temos

$$
t=\frac{\sum_{i=1}^{m} a_{i} b_{i}}{\sum_{j=m+1}^{s} a_{j} b_{j}}
$$

onde $a_{i}, a_{j} \in K^{\prime}$ e $b_{i}, b_{j} \in F$. Façamos $K_{1}=K\left(a_{1}, \ldots, a_{s}\right)$. Logo, $\left[K_{1}: K\right]<\infty$, e temos $t \in F_{1}=F K_{1}$. Seja $P_{1}=P^{\prime} \cap F_{1} \in \mathbb{P}\left(F_{1}\right)$. Então,

$$
1=v_{P^{\prime}}(t)=e\left(P^{\prime} \mid P_{1}\right) \cdot v_{P_{1}}(t) \text {. }
$$

Logo, $v_{P_{1}}(t)=1$ e $e\left(P^{\prime} \mid P_{1}\right)=1$. Como $\left[K_{1}: K\right]<\infty$, pelo caso finito provado anteriormente, temos $e\left(P_{1} \mid P\right)=1$. Logo, $e\left(P^{\prime} \mid P\right)=e\left(P^{\prime} \mid P_{1}\right) \cdot e\left(P_{1} \mid P\right)=1$.

Proposição 3.47. Seja $F / K$ um corpo de funções com corpo de constantes $K$. Suponha que $F^{\prime} / F$ é uma extensão finita com corpo de constantes $K^{\prime}$. Seja $\bar{K} \subseteq \Phi$ o fecho algébrico de K. Então:

$$
\left[F^{\prime}: F\right]=\left[F^{\prime} \bar{K}: F \bar{K}\right] \cdot\left[K^{\prime}: K\right] \text {. }
$$

No caso especial em que $F^{\prime}=F(y)$, e $\varphi(T) \in F[T]$ é o polinômio minimal de $y$ sobre $F$, são equivalentes:

(1) $K^{\prime}=K$;

(2) $\varphi(T)$ é (absolutamente) irredutível em $F \bar{K}[T]$.

Demonstração. Como $F \subseteq F K^{\prime} \subseteq F^{\prime}$ e $F^{\prime} / F$ é finito, temos

$$
\text { (I) } \quad\left[F^{\prime}: F\right]=\left[F^{\prime}: F K^{\prime}\right] \cdot\left[F K^{\prime}: F\right] \text {. }
$$

Temos ainda

$$
\left[F^{\prime}: F\right]<\infty \Longrightarrow\left[K^{\prime}: K\right]<\infty,
$$

e, como $K^{\prime} / K$ é separável, temos $K^{\prime}=K(\alpha), \alpha \in K^{\prime}$, e, conseqüentemente, $F K^{\prime}=F(\alpha)$. Logo, pelo lema 3.46, temos

$$
\text { (II) } \quad[F(\alpha): F]=[K(\alpha): K] \Longrightarrow\left[F K^{\prime}: F\right]=\left[K^{\prime}: K\right] \text {. }
$$

Como $F \bar{K} / \bar{K}$ é extensão algébrica constante de $F K^{\prime} / K^{\prime}$, e $F^{\prime} \bar{K} / \bar{K}$ é extensão algébrica constante de $F^{\prime} / K^{\prime}$, o item (c) da proposição $3.45 \mathrm{diz}$ que, para $x \in F \backslash K$, temos $\left[F K^{\prime}: K^{\prime}(x)\right]=[F \bar{K}: \bar{K}(x)]$ e $\left[F^{\prime}: K^{\prime}(x)\right]=\left[F^{\prime} \bar{K}: \bar{K}(x)\right]$, e, conseqüentemente,

(III) $\quad\left[F^{\prime}: F K^{\prime}\right]=\left[F^{\prime} \bar{K}: F \bar{K}\right]$. 
Substituindo (III) e (II) em (I), obtemos

$$
\text { (IV) } \quad\left[F^{\prime}: F\right]=\left[F^{\prime} \bar{K}: F \bar{K}\right] \cdot\left[K^{\prime}: K\right] \text {. }
$$

O diagrama abaixo auxilia no processo acima:

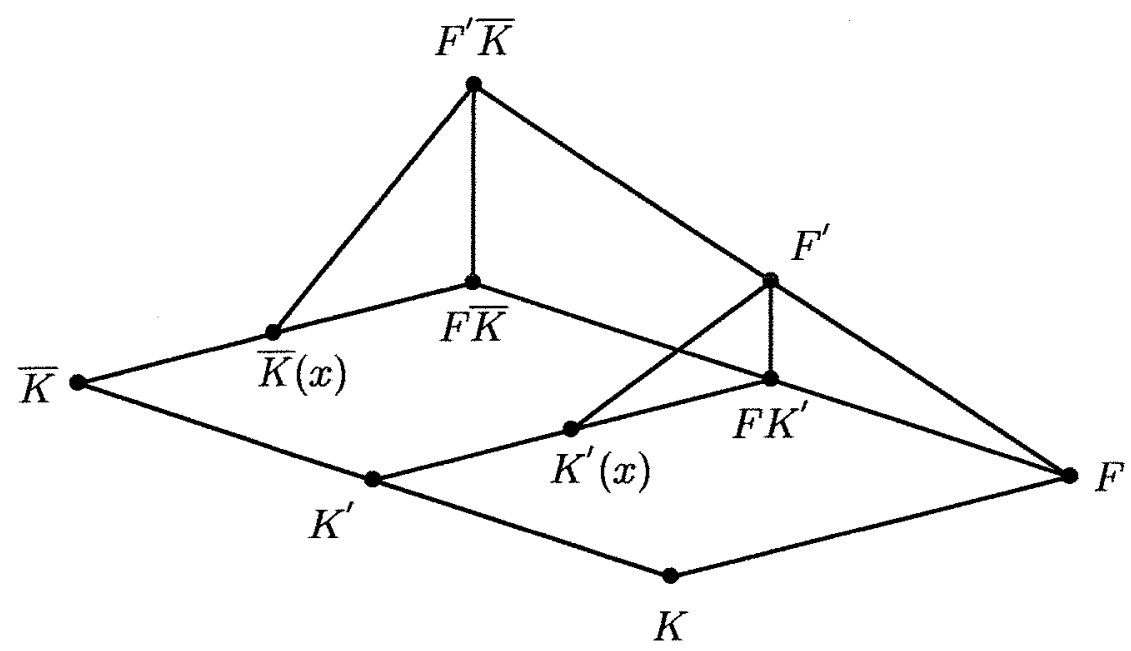

Agora, mostraremos as equivalências.

- (1) $\Longrightarrow$ (2) Como $F^{\prime}=F(y)$, temos $F^{\prime} \bar{K}=F(y) \bar{K}=F \bar{K}(y)$. Logo, por (IV), se $\left[K^{\prime}: K\right]=1$, temos

$$
\operatorname{deg}(\varphi(T))=\left[F^{\prime}: F\right]=\left[F^{\prime} \bar{K}: F \bar{K}\right]=[F \bar{K}(y): F \bar{K}] .
$$

Portanto, $\varphi(T)$ é irredutível sobre $F \bar{K}$.

- $(2) \Longrightarrow(1)$ É o caminho inverso.

\subsection{Extensões de Galois}

Uma extensão $F^{\prime} / K^{\prime}$ de $F / K$ é dita de Galois se $F^{\prime} / F$ é uma extensão de Galois finita, isto é, o grupo de automorfismos

$$
\operatorname{Aut}\left(F^{\prime} / F\right)=\left\{\sigma: F^{\prime} \longrightarrow F^{\prime}: \sigma \text { é um } F \text {-isomorfismo }\right\}
$$

tem ordem $\left[F^{\prime}: F\right]$.

Seja $P \in \mathbb{P}(F)$. Então, o grupo de Galois de $F^{\prime} / F$ age no conjunto das extensões $\left\{P^{\prime} \in \mathbb{P}\left(F^{\prime}\right): P^{\prime} \mid P\right\}$ via $\sigma\left(P^{\prime}\right)=\left\{\sigma(x): x \in P^{\prime}\right\}$. Além disso, no lema 3.39, vimos que a valorização correspondente $v_{\sigma\left(P^{\prime}\right)}$ é dada por

$$
v_{\sigma\left(P^{\prime}\right)}(y)=v_{P^{\prime}}\left(\sigma^{-1}(y)\right), \forall y \in F^{\prime} .
$$


Teorema 3.48. Seja $F^{\prime} / K^{\prime}$ uma extensão de Galois de $F / K$, e $P_{1}, P_{2} \in \mathbb{P}\left(F^{\prime}\right)$ extensôes de $P \in \mathbb{P}(F)$. Então, $P_{2}=\sigma\left(P_{1}\right)$ para algum $\sigma \in A u t\left(F^{\prime} / F\right)$, ou seja, o grupo de Galois age transitivamente no conjunto das extensöes de $P$.

Demonstração. Suponhamos que $\sigma\left(P_{1}\right) \neq P_{2}$ para todo $\sigma \in G:=\operatorname{Aut}\left(F^{\prime} / F\right)$. Pelo teorema 2.22 (da aproximação fraca), existe $z \in F^{\prime}$ tal que $v_{P_{2}}(z)>0$ e $v_{Q}(z)=0$, qualquer que seja $Q \in \mathbb{P}\left(F^{\prime}\right)$, com $Q \mid P$ e $Q \neq P_{2}$. Seja $N_{F^{\prime} / F^{\prime}}: F^{\prime} \longrightarrow F$ a função norma. Pelas propriedades desta função, sabemos que:

$$
N_{F^{\prime} / F}(z)=\prod_{\sigma \in G} \sigma(z)
$$

o que implica que

$$
\begin{aligned}
v_{P_{1}}\left(N_{F^{\prime} / F}(z)\right) & =\sum_{\sigma \in G} v_{P_{1}}(\sigma(z)) \\
& =\sum_{\sigma \in G} v_{\sigma^{-1}\left(P_{1}\right)}(z) \\
& =\sum_{\sigma \in G} v_{\sigma\left(P_{1}\right)}(z)=0
\end{aligned}
$$

pois $\sigma\left(P_{1}\right) \neq P_{2}$, qualquer que seja $\sigma \in G$.

Por outro lado, temos

$$
v_{P_{2}}\left(N_{F^{\prime} / F}(z)\right)=\sum_{\sigma \in G} v_{\sigma\left(P_{2}\right)}(z)>0 \quad(\star),
$$

pois $v_{P_{2}}(z)>0$ e, se $\sigma\left(P_{2}\right) \neq P_{2}$, temos $v_{\sigma\left(P_{2}\right)}(z)=0$.

Mas, $N_{F^{\prime} / F}(z)=y \in F$; logo,

$$
v_{P_{1}}(y)=0 \Longleftrightarrow v_{P}(y)=0 \Longleftrightarrow v_{P_{2}}(y)=0,
$$

o que contradiz $(\star)$.

Corolário 3.49. Usaremos a notação do teorema anterior (em particular, $F^{\prime} / F$ é galoisiana). Sejam $P_{1}, \ldots, P_{r}$ todas as extensões de $F^{\prime}$ sobre $P$. Então, temos:

(a) $e\left(P_{i} \mid P\right)=e\left(P_{j} \mid P\right)$ e $f\left(P_{i} \mid P\right)=f\left(P_{j} \mid P\right)$ para todo $i, j$; portanto, abreviaremos $e(P):=e\left(P_{i} \mid P\right)$ e $f(P):=f\left(P_{i} \mid P\right)$, e chamaremos $e(P)$ de índice de ramificação e $f(P)$ de grau relativo de $P$;

(b) $e(P) \cdot f(P) \cdot r=\left[F^{\prime}: F\right]$;

(c) os expoentes da diferente $d\left(P_{i} \mid P\right)$ e $d\left(P_{j} \mid P\right)$ são os mesmos para todo $i, j$.

Demonstração. (a) Pelo item (c) do lema 3.39, sabemos que

$$
e\left(\sigma\left(P_{i}\right) \mid P\right)=e\left(P_{i} \mid P\right) \quad \text { e } \quad f\left(\sigma\left(P_{i}\right) \mid P\right)=f\left(P_{i} \mid P\right)
$$


Então, dados $P_{i}$ e $P_{j}$ extensões de $P$ em $F^{\prime}$, tomamos $\sigma \in G$ tal que $\sigma\left(P_{i}\right)=P_{j}$, e assim:

$$
e\left(P_{i} \mid P\right)=e\left(\sigma\left(P_{i}\right) \mid P\right)=e\left(P_{j} \mid P\right)
$$

e

$$
f\left(P_{i} \mid P\right)=f\left(\sigma\left(P_{i}\right) \mid P\right)=f\left(P_{j} \mid P\right) .
$$

(b) Sejam $P_{1} \ldots, P_{r}$ os lugares de $F^{\prime}$ sobre $P$. Então:

$$
\left[F^{\prime}: F\right]=\sum_{i=1}^{r} e_{i} f_{i}=\sum_{i=1}^{r} e(P) f(P)=e(P) \cdot f(P) \cdot r .
$$

(c) Consideremos o fecho inteiro

$$
O_{P}^{\prime}=\bigcap_{i=1}^{r} O_{P_{i}}
$$

de $O_{P}$ em $F^{\prime}$, e o módulo complementar

$$
\mathfrak{C}_{P}=\left\{z \in F^{\prime}: \operatorname{tr}_{F^{\prime} / F}\left(z \cdot O_{P}^{\prime}\right) \subseteq O_{P}\right\}
$$

Seja $\sigma \in G$. Podemos ver que $\sigma\left(O_{P}^{\prime}\right)=O_{P}^{\prime}$, pois $P_{i}\left|P \Rightarrow \sigma\left(P_{i}\right)\right| P$. Logo:

$$
\sigma\left(O_{P}^{\prime}\right)=\cap \sigma\left(O_{P_{i}}\right)=\cap O_{\sigma\left(P_{i}\right)}=O_{P}^{\prime} .
$$

Usando o fato que $\operatorname{tr}_{F^{\prime} / F}(u)=\operatorname{tr}_{F^{\prime} / F}(\sigma(u))$ para todo $u \in F^{\prime}$, é possível mostrar que $\sigma\left(\mathfrak{C}_{P}\right)=\mathfrak{C}_{P}$

Assim, escrevendo $\mathfrak{C}_{P}=t \cdot O_{P}^{\prime}$, temos $\mathfrak{C}_{P}=\sigma(t) \cdot O_{P}^{\prime}$. Então, pela definição 3.32 de expoente da diferente, e pelo item (c) da proposição 3.31 , temos

$$
-d\left(P_{i} \mid P\right)=v_{P_{i}}(t)=v_{P_{i}}(\sigma(t))
$$

para todo $1 \leq i \leq r$. Sejam $P_{i}$ e $P_{j}$ extensões de $P$ em $F^{\prime}$. Tomemos $\sigma \in G$ tal que $\sigma\left(P_{j}\right)=P_{i}$. Então:

$$
-d\left(P_{i} \mid P\right)=v_{P_{i}}(\sigma(t))=v_{\sigma^{-1}\left(P_{i}\right)}(t)=v_{P_{j}}(t)=-d\left(P_{j} \mid P\right) .
$$

Trataremos aqui de um tipo específico de extensão de Galois de corpos de funções, que chamaremos de extensões de Artin-Schreier.

A definição de extensão de Artin-Schreier "clássica" pode ser dada da seguinte forma:

Seja $F / K$ uma extensão de corpos de característica $p>0$. Suponha que $u \in F$ satisfaz $u \neq \omega^{p}-\omega$, para todo $\omega \in F$. Seja $F^{\prime}=F(y)$, onde $y^{p}-y=u$. Tal extensão $F^{\prime} / F$ é chamada de extensão de Artin-Schreier de F. 
No entanto, para contemplar o Capítulo 4, trataremos de uma extensão (na próxima proposição) a qual também chamaremos de Artin-Schreier, mas que possui algumas modificações em relação à clássica enunciada acima. Na verdade, ambos são casos particulares de uma generalização que pode ser encontrada em [11] (página 117).

Proposição 3.50. Seja $q$ uma potência de um primo $p$, e $F / K$ um corpo de funções algébricas sobre $K$, onde $K=\mathbb{F}_{q^{2}}$ é algebricamente fechado em $F$. Seja $\omega \in F$ e suponha que existe um lugar $P \in \mathbb{P}(F)$ tal que

$$
v_{P}(\omega)=-m, m>0, \quad e \quad m d c(m, q)=1 .
$$

Então, o polinômio $\varphi(T)=T^{q}+T-\omega \in F[T]$ é absolutamente irredutível, isto é, irredutivel em $\bar{K} \cdot F[T]$, onde $\bar{K} \cdot F$ denota o compósito do fecho algébrico de $K$ e $F$.

Seja $E=F(z)$, onde $\varphi(z)=0$, isto é, $z^{q}+z=\omega$. Então:

(i) $E / F$ é uma extensão de Galois de grau $[E: F]=q$, o grupo de Galois é abeliano elementar de expoente $p$, e $K$ é algebricamente fechado em $E$;

(ii) o lugar $P$ é totalmente ramificado em $E$, isto é, existe um único lugar $P^{\prime} \in \mathbb{P}(E)$ tal que $P^{\prime} \mid P$ e e $\left(P^{\prime} \mid P\right)=q$; temos ainda que $\operatorname{deg}\left(P^{\prime}\right)=\operatorname{deg}(P)$, e o expoente da diferente de $P^{\prime} \mid P$ é dado por:

$$
d\left(P^{\prime} \mid P\right)=(q-1)(m+1)
$$

(iii) seja $R \in \mathbb{P}(F)$, e suponhamos que $v_{R}\left(\omega-\left(u^{q}+u\right)\right) \geq 0$ para algum $u \in F$; então, $R$ é nấo-ramificado em $E / F$; isto ocorre em particular se $v_{R}(\omega) \geq 0$;

(iv) seja $Q \in \mathbb{P}(F)$ um zero de $\omega-\gamma$, com $\gamma \in \mathbb{F}_{q} ;$ então, a equação $\alpha^{q}+\alpha=\gamma$ tem $q$ raízes distintas $\alpha \in K$, e para cada $\alpha$ existe um único lugar $Q_{\alpha} \in \mathbb{P}(E)$ tal que $Q_{\alpha} \mid Q$ e $Q_{\alpha}$ é zero de $z-\alpha$.

Demonstração. - Usando a notação da proposição 3.14 , segue imediatamente que $T^{q}+T-\omega \in F[T]$ é irredutível, e que $P \in \mathbb{P}(F)$ é totalmente ramificado em $E$.

- Se $z \in E$ é uma raiz de $\varphi(T)$, então

$$
\varphi(T)=\prod_{i=1}^{q}\left(T-z-\alpha_{i}\right),
$$

onde $\alpha_{i} \in \mathbb{F}_{q^{2}}$ e $\alpha_{i}^{q}+\alpha_{i}=0$. Logo, $E$ é corpo de raízes de $\varphi(T)$, e como $\varphi(T)$ é separável, temos que $E / F$ é uma extensão de Galois de grau $q$.

- Os automorfismos $E / F$ são dados por $\sigma_{i}(z)=z+\alpha_{i}$, com $\alpha_{i} \in \mathbb{F}_{q^{2}}$ e $\alpha_{i}^{q}+\alpha_{i}=0$ para $i=1, \ldots, q$, ou seja, o grupo de Galois de $E / F$ é isomorfo ao grupo aditivo $G=\left\{\alpha \in K: \alpha^{q}+\alpha=0\right\}, \mathrm{e}, \operatorname{como} \operatorname{car}(K)=p$, temos que $\exp (G)=m m c\{o(g)$ : $g \in G\}=p$, e portanto $\operatorname{Gal}(E / F)$ é abeliano elementar de expoente $p$. 
- Provaremos agora que $K$ é algebricamente fechado em $E$, isto é, se $K^{\prime} \supseteq K$ é o corpo das constantes de $E$, então $K^{\prime}=K$.

Seja $P^{\prime} \in \mathbb{P}(E)$ a única extensão de $P \in \mathbb{P}(F)$ em $E$. Suponhamos que $\left[K^{\prime}: K\right]>1$, e consideremos o corpo intermediário $F_{1}=F K^{\prime} \supsetneq F$, e o lugar $Q_{1}=P^{\prime} \cap F_{1}$, o único lugar sobre $P$ em $F_{1}$. Logo, $e\left(Q_{1} \mid P\right)=\left[F_{1}: F\right]$, e, como $F_{1} \neq F$, temos $e\left(Q_{1} \mid P\right)>1$. Mas, por outro lado, pelo item (d) da proposição 3.45 , temos $e\left(Q_{1} \mid P\right)=1$. Esta contradição mostra que $K^{\prime}=K$.

- Agora, sendo $K$ algebricamente fechado em $E$, segue, da proposição 3.47 , que $\varphi(T)$ é absolutamente irredutível em $F[T]$, e ainda que, se $P^{\prime} \in \mathbb{P}(E)$ é a única extensão de $P$ em $E$, então $\operatorname{deg}\left(P^{\prime}\right)=\operatorname{deg}(P)$, pois

$$
\operatorname{deg}\left(P^{\prime}\right)=\left[\mathbb{F}_{P^{\prime}}: K^{\prime}\right]=\left[\mathbb{F}_{P^{\prime}}: K\right]=f\left(P^{\prime} \mid P\right) \cdot\left[\mathbb{F}_{P}: K\right]=\operatorname{deg}(P)
$$

- Provaremos agora que $d\left(P^{\prime} \mid P\right)=(q-1)(m+1)$.

Como $z^{q}+z=\omega$ e $v_{P}(\omega)=-m$, temos

$$
\begin{aligned}
v_{P^{\prime}}\left(z^{q}+z\right)=v_{P^{\prime}}(\omega)=e\left(P^{\prime} \mid P\right) \cdot v_{P}(\omega)=-q \cdot m & \Longrightarrow v_{P^{\prime}}\left(z^{q}\right)=-q \cdot m \\
& \Longrightarrow v_{P^{\prime}}(z)=-m .
\end{aligned}
$$

Seja $x \in F$ tal que $v_{P}(x)=1$. Tomemos $i, j \geq 0$ tais que $1=i \cdot q-m \cdot j$ (isto é possível, pois $m d c(m, q)=1$ ). Então, o elemento $t=x^{i} \cdot z^{j}$ é um elemento primo de $P^{\prime}$. De fato:

$$
v_{P^{\prime}}(t)=i \cdot v_{P^{\prime}}(x)+j \cdot v_{P^{\prime}}(z)=i \cdot q \cdot v_{P}(x)+j \cdot(-m)=i \cdot q-m \cdot j=1 .
$$

Pela proposição 3.44 , o expoente da diferente $d\left(P^{\prime} \mid P\right)$ é dado por

$$
d\left(P^{\prime} \mid P\right)=v_{P^{\prime}}\left(\psi^{\prime}(t)\right),
$$

onde $\psi(T) \in F[T]$ é o polinômio minimal de $t$ sobre $F$. Iremos agora em direção ao cálculo de $v_{P^{\prime}}\left(\psi^{\prime}(t)\right)$.

Seja $G:=\operatorname{Gal}(E / F)$ o grupo de Galois de $E / F$. Como $t=x^{i} \cdot z^{j}$, temos que $\sigma(t)=x^{i} \cdot(\sigma(z))^{j}$ é raiz de $\psi(T)$, e, como $\{\sigma(z): \sigma \in G\}$ são todas as raízes distintas de $T^{q}+T-\omega$, temos que $\{\sigma(t): \sigma \in G\}$ são todas as raízes de $\psi(T)$, pois, se não fossem, então existiriam $\sigma_{1}, \sigma_{2} \in G$ distintos tais que $\left(\sigma_{1}(z)\right)^{j}=\left(\sigma_{2}(z)\right)^{j}$. Mas, como $\sigma_{1}(z)$ e $\sigma_{2}(z)$ são raízes distintas de $T^{q}+T-\omega$, temos que $\sigma_{1}(z)+\alpha=\sigma_{2}(z)$, para algum $\alpha \in K$, com $\alpha^{q-1}=-1$, e assim teríamos $\left(\sigma_{1}(z)\right)^{j}=\left(\sigma_{1}(z)+\alpha\right)^{j}$, o que é absurdo, pois $K$ é algebricamente fechado em $E$.

Desta forma, temos

$$
\psi(T)=\prod_{\sigma \in G}(T-\sigma(t))=(T-t) \cdot h(T),
$$

com

$$
h(T)=\prod_{\sigma \neq i d}(T-\sigma(t)) \in E[T]
$$


Assim, $\psi^{\prime}(T)=h(T)+(T-t) \cdot h^{\prime}(T)$ e $\psi^{\prime}(t)=h(t)$, de onde concluímos que

$$
d\left(P^{\prime} \mid P\right)=v_{P^{\prime}}\left(\prod_{\sigma \neq i d}(t-\sigma(t))\right)=\sum_{\sigma \neq i d} v_{P^{\prime}}(t-\sigma(t)) .
$$

Como $\sigma \in G \backslash\{i d\}$ é da forma $\sigma(z)=z+\alpha$, onde $\alpha \in \mathbb{F}_{q^{2}}$ é tal que $\alpha^{q-1}=-1$, temos

$$
\begin{aligned}
t-\sigma(t) & =x^{i} \cdot z^{j}-x^{i} \cdot(z+\alpha)^{j} \\
& =x^{i} \cdot\left(z^{j}-\sum_{l=0}^{j}\left(\begin{array}{l}
j \\
l
\end{array}\right) z^{j-l} \cdot \alpha^{l}\right) \\
& =-x^{i} \cdot \sum_{l=1}^{j}\left(\begin{array}{l}
j \\
l
\end{array}\right) z^{j-l} \cdot \alpha^{l} .
\end{aligned}
$$

Como $v_{P^{\prime}}(z)<0$, temos $v_{P^{\prime}}\left(z^{j-1}\right)<v_{P^{\prime}}\left(z^{j-l}\right)$, com $l \geq 2$, e, pela desigualdade triangular estrita, obtemos

$$
\begin{aligned}
v_{P^{\prime}}(t-\sigma(t)) & =v_{P^{\prime}}\left(x^{i}\right)+v_{P^{\prime}}\left(z^{j-1}\right) \\
& =i \cdot v_{P^{\prime}}(x)+(j-1) \cdot v_{P^{\prime}}(z) \\
& =i \cdot p+(j-1) \cdot(-m) \\
& =i \cdot p-j \cdot m+m \\
& =1+m .
\end{aligned}
$$

Então:

$$
d\left(P^{\prime} \mid P\right)=\sum_{\sigma \neq i d} v_{P^{\prime}}(t-\sigma(t))=(q-1) \cdot(m+1)
$$

- Agora, provaremos (iii). Assumindo que $v_{R}\left(\omega-\left(u^{q}+u\right)\right) \geq 0$, façamos $z_{1}=z-u$ e $\omega_{1}=\omega-\left(u^{q}+u\right)$, e temos $E=F\left(z_{1}\right)$ e $\varphi_{1}(T)=T^{q}+T-\omega_{1}$ é o polinômio minimal de $z_{1}$.

Como $v_{R}\left(\omega_{1}\right) \geq 0$, temos que $z_{1}$ é inteiro sobre $O_{R}$, e o expoente da diferente $d\left(R^{\prime} \mid R\right)$ de qualquer extensão $R^{\prime}$ de $R$ satisfaz

$$
0 \leq d\left(R^{\prime} \mid R\right) \leq v_{R^{\prime}}\left(\varphi_{1}^{\prime}\left(z_{1}\right)\right)=0
$$

pois basta observar que $\varphi_{1}^{\prime}(T)=1$ e usar o teorema 3.43 .

Portanto, $d\left(R^{\prime} \mid R\right)=0$. Logo, $R^{\prime} \mid R$ é não-ramificado, pelo teorema 3.37 da diferente de Dedekind.

- Finalmente, vamos provar (iv). Seja $\omega_{1}=\omega-\gamma$ e $z_{1}=z-\alpha$, onde $\alpha \in K$ é tal que $\alpha^{q}+\alpha=\gamma$. Temos assim que $E=F\left(z_{1}\right)$ e $\varphi_{1}(T)=T^{q}+T-\omega_{1}$ é o polinômio minimal de $z_{1}$ sobre $F$ (pois, se não fosse, teríamos um polinômio em $F[T]$ de grau menor que $q$ anulando $z$ ). Agora, invocamos o teorema 3.29 (de Kummer), 
e usaremos sua notação. Como $Q \in \mathbb{P}(F)$ é zero de $\omega_{1}$, temos que $z_{1}$ é inteiro sobre $O_{Q}$. Logo,

$$
\bar{\varphi}_{1}(T)=T^{q}+T=\prod_{i=1}^{q}\left(T-\bar{\alpha}_{i}\right),
$$

onde $\alpha_{i} \in \mathbb{F}_{q^{2}}$ e $\alpha_{i}^{q}+\alpha_{i}=0$ (observe que, na notação do teorema 3.29 , temos $\epsilon_{i}=1$, para $i=1, \ldots, q$ ). Tomando $\gamma_{i}(T):=T-\alpha_{i} \in O_{Q}[T]$ (observe que $\bar{\gamma}_{i}(T)=T-\bar{\alpha}_{i}$ ) para cada $1 \leq i \leq q$, temos:

- um único lugar $Q_{\alpha_{i}} \in \mathbb{P}(E)$, com $Q_{\alpha_{i}} \mid Q$;

○ $f\left(Q_{\alpha_{i}} \mid Q\right)=1 \Longrightarrow \operatorname{deg}\left(Q_{\alpha_{i}}\right)=\operatorname{deg}(Q)$;

○ $Q_{\alpha_{i}}$ é zero de $z_{1}-\alpha_{i}$.

Mas, como $z_{1}=z-\alpha$ para algum $\alpha \in K, \operatorname{com} \alpha^{q}+\alpha=\gamma$, temos $z_{1}-\alpha_{i}=z-\left(\alpha+\alpha_{i}\right)$, onde $\left(\alpha+\alpha_{i}\right)^{q}+\left(\alpha+\alpha_{i}\right)=\gamma$. Então, sem perda de generalidade, podemos denotar $\alpha:=\alpha+\alpha_{i}$, e afirmar que $Q_{\alpha_{i}}$ é zero de $z-\alpha_{i}$.

\subsection{As Propriedades $P_{i}$}

Nesta seção, reunimos alguns resultados que visam simplificar as demonstrações que surgirão no próximo Capítulo . Tais resultados serão denominados propriedades $P_{i}$. No que segue, denotaremos por $F / K$ um corpo de funções de característica $p>0$, enquanto que $q$ denotará uma potência de $p$.

Definição 3.51. - Sejam $x, y, z \in F$ e $P \in \mathbb{P}(F)$. Escrevemos

$$
x=y+\mathcal{O}(z) \text { em } P
$$

se $x=y+t z$, com $v_{P}(t) \geq 0$, isto é, $t \in O_{P}$ (ou, equivalentemente, $\left.v_{P}\left(\frac{x-y}{z}\right) \geq 0\right)$. Em particular,

$$
x=y+\mathcal{O}(1) \text { em } P \Longrightarrow v_{P}(x-y) \geq 0 .
$$

- $\quad$ operador de Artin-Schreier generalizado $\wp: F \longrightarrow F$ é definido por $\wp(u)=$ $u^{q}+u$.

Lema 3.52. Seja $F / K$ um corpo de funções algébricas de característica $p>0$. Usando a notação da definição acima, temos:

- $P_{0}: x=a \cdot \mathcal{O}(b) \Longrightarrow x=\mathcal{O}(a b)$.

- $\boldsymbol{P}_{1}: \gamma \in \mathbb{F}_{q} \Longrightarrow \gamma \cdot \wp(u)=\wp(\gamma u)$.

- $\boldsymbol{P}_{\mathbf{2}}: \wp(u)=\mathcal{O}(1) \Longrightarrow u=\mathcal{O}(1)$.

- $\boldsymbol{P}_{\mathbf{3}}$ : Se $m, n$ são inteiros tais que $m \leq n$ e $a=\mathcal{O}\left(x^{m}\right)+\mathcal{O}\left(x^{n}\right), \operatorname{com} v_{P}(x) \geq 0$, então $a=\mathcal{O}\left(x^{m}\right)$. 
- $\boldsymbol{P}_{4}:$ Se $m, n$ são inteiros tais que $m \leq n$ e $a=x^{m}+\mathcal{O}\left(x^{n}\right)$, com $v_{P}(x) \geq 0$, então $a=\mathcal{O}\left(x^{m}\right)$.

- $\boldsymbol{P}_{\mathbf{5}}$ : Se $m, n$ são inteiros tais que $m \leq n$ e $a=\mathcal{O}\left(x^{n}\right)$, com $v_{P}(x) \geq 0$, então $a=\mathcal{O}\left(x^{m}\right)$.

- $\boldsymbol{P}_{\mathbf{6}}$ : Se $a=1-y^{q-1}+\mathcal{O}\left(y^{q}\right)$, onde $q$ é potência de $p$ e $v_{P}(y)>0$, então $a^{-1}=$ $1+y^{q-1}+\mathcal{O}\left(y^{q}\right)$.

- $\boldsymbol{P}_{7}$ : Se $a=1+y^{q-1}+\mathcal{O}\left(y^{q}\right)$, onde $q$ é potência de p e $v_{P}(y)>0$, então $a^{q+1}=$ $1+y^{q-1}+\mathcal{O}\left(y^{q}\right)$.

- $\boldsymbol{P}_{\mathbf{8}}:$ Se $a=\gamma+\mathcal{O}(y)$, onde $\gamma \in \mathbb{F}_{q^{2}} \backslash\{0\}$ e $v_{P}(y)>0$, então $a^{-1}=\gamma^{-1}+\mathcal{O}(y)$.

Demonstração. $\boldsymbol{P}_{\mathbf{0}}$ : De fato:

$$
x=a \cdot(b t), v_{P}(t) \geq 0 \Longrightarrow x=(a b) \cdot t \Longrightarrow x=\mathcal{O}(a b) .
$$

$\boldsymbol{P}_{1}:$ De fato, temos que:

$$
\begin{aligned}
\gamma \in \mathbb{F}_{q} & \Longrightarrow \gamma^{q}=\gamma \\
& \Longrightarrow \gamma \cdot \wp(u)=\gamma \cdot u^{q}+\gamma \cdot u=(u \gamma)^{q}+(u \gamma)=\wp(u \gamma)
\end{aligned}
$$

$\boldsymbol{P}_{2}:$ De fato, suponhamos que $u \neq \mathcal{O}(1)$, isto é, $v_{P}(u)<0$. Então:

$$
\begin{aligned}
v_{P}\left(u^{q}\right)<v_{P}(u) & \Longrightarrow 0>v_{P}\left(u^{q}\right)=\min \left\{v_{P}\left(u^{q}\right), v_{P}(u)\right\}=v_{P}\left(u^{q}+u\right) \\
& \Longrightarrow \wp(u) \neq \mathcal{O}(1),
\end{aligned}
$$

contradição.

$\boldsymbol{P}_{\mathbf{3}}:$ De fato, se $a=t_{1} x^{m}+t_{2} x^{n}$, onde $t_{1}, t_{2} \in O_{P}$, então

$$
a=x^{m}\left(t_{1}+t_{2} x^{n-m}\right) \text {. }
$$

Mas, como $t_{1}, t_{2} \in O_{P}$ e $n-m \geq 0$, temos que $t_{1}+t_{2} x^{n-m} \in \mathcal{O}_{P}$. Logo, $a=\mathcal{O}\left(x^{m}\right)$.

$\boldsymbol{P}_{4}$ : De fato, como $v_{P}(1) \geq 0$, temos que:

$$
x^{m}=1 \cdot x^{m} \Longrightarrow x^{m}=\mathcal{O}\left(x^{m}\right) \Longrightarrow a=\mathcal{O}\left(x^{m}\right)+\mathcal{O}\left(x^{n}\right),
$$

e, pela propriedade $P_{3}$, segue que $a=\mathcal{O}\left(x^{m}\right)$.

$\boldsymbol{P}_{\mathbf{5}}:$ De fato:

$$
a=t \cdot x^{n}, v_{P}(t) \geq 0 \Longrightarrow a=\left(t \cdot x^{n-m}\right) x^{m} \Longrightarrow a=\mathcal{O}\left(x^{m}\right),
$$

pois $v_{P}\left(t \cdot x^{n-m}\right) \geq 0$.

$\boldsymbol{P}_{6}$ : Com efeito, sabemos que:

$$
a=1-y^{q-1}+\mathcal{O}\left(y^{q}\right) \Longrightarrow a=1-y^{q-1}+y^{q} t
$$


onde $t \in O_{P}$. Assim, temos que:

$$
a^{-1}=\frac{1}{1-y^{q-1}+y^{q} t}=1+y^{q-1}+y^{q} \cdot\left(\frac{y^{q-2}-t y^{q-1}-t}{1-y^{q-1}+y^{q} t}\right) .
$$

Como $v_{P}\left(1-y^{q-1}+y^{q} t\right)=\min \left\{v_{P}(1), v_{P}\left(-y^{q-1}+y^{q} t\right)\right\}=0$, segue que:

$$
v_{P}\left(\frac{y^{q-2}-t y^{q-1}-t}{1-y^{q-1}+y^{q} t}\right)=v_{P}\left(y^{q-2}-t y^{q-1}-t\right) \geq 0
$$

pois todas as parcelas estão em $O_{P}$. Portanto, $a^{-1}=1+y^{q-1}+\mathcal{O}\left(y^{q}\right)$.

$\boldsymbol{P}_{7}$ : Com efeito, temos que:

$$
a^{q+1}=\left(1+y^{q-1}+y^{q} t\right) \cdot\left(1+y^{q-1}+y^{q} t\right)^{q},
$$

onde $v_{P}(t) \geq 0$. Logo:

$$
\begin{aligned}
a^{q+1} & =\left(1+y^{q-1}+y^{q} t\right) \cdot\left(1+y^{q(q-1)}+y^{q^{2}} t^{q}\right) \\
& =1+y^{q-1}+y^{q} t+\left(y^{q^{2}-q}+y^{q^{2}} t^{q}\right)\left(1+y^{q-1}+y^{q} t\right) \\
& =1+y^{q-1}+y^{q} \cdot[\underbrace{t+\left(y^{q^{2}-2 q}+y^{q^{2}-q} t^{q}\right)\left(1+y^{q-1}+y^{q} t\right)}_{(*)}] \\
& =1+y^{q-1}+\mathcal{O}\left(y^{q}\right),
\end{aligned}
$$

pois (*) está em $O_{P}$, uma vez que temos produtos e somas de elementos de $O_{P}$.

$\boldsymbol{P}_{\mathbf{8}}$ : De fato, temos que:

$$
a=\gamma+\mathcal{O}(y) \Longrightarrow a=\gamma+y t
$$

onde $v_{P}(t) \geq 0$. Logo:

$$
a^{-1}=\frac{1}{\gamma+y t}=\gamma^{-1}+y \cdot\left(\frac{-t \gamma^{-1}}{\gamma+y t}\right) .
$$

Como $v_{P}(\gamma+y t)=\min \left\{v_{P}(\gamma), v_{P}(y t)\right\}=0$, temos que:

$$
v_{P}\left(\frac{-t \gamma^{-1}}{\gamma+y t}\right)=v_{P}\left(-t \gamma^{-1}\right) \geq 0
$$

Portanto, $a^{-1}=\gamma^{-1}+\mathcal{O}(y)$. 


\section{Capítulo 4}

\section{A Torre de Corpos $F_{n} / K$}

O nosso propósito é construir uma torre de corpos de funções, usando extensões de Artin-Schreier, que atinja a cota de Drinfeld-Vladut.

Uma vez construída a torre $F_{n} / K$, as etapas seguintes culminam no teorema 4.17 , onde calculamos o gênero $g_{n}$ de $F_{n} / K$, e na proposição 4.19 , onde obtemos uma estimativa do número $N_{n}$ de lugares de grau 1 de $F_{n} / K$, suficiente para atingir a cota desejada.

Gostaríamos de ressaltar que a última seção deste capítulo contém a nossa contribuição a este trabalho. Nela, estabelecemos a quantidade efetiva $N_{n}$ de lugares de grau $1 \mathrm{em}$ $F_{n} / K$. Os cálculos apresentados para a obtenção de $N_{n}$ são de nossa autoria, seguindo sugestões do final de [3].

\subsection{Construindo a Torre}

Definição 4.1. Seja $F_{1}=F_{q^{2}}\left(x_{1}\right)$ um corpo de funções racionais, onde $q \geq 2$ é potência de um primo $p$. Para $n \geq 1$, definiremos $F_{n+1}:=F_{n}\left(z_{n+1}\right)$, onde $z_{n+1}$ é tal que

$$
z_{n+1}^{q}+z_{n+1}=x_{n}^{q+1}
$$

com

$$
x_{n}:=\frac{z_{n}}{x_{n-1}} \in F_{n} \quad \text { para } n \geq 2 .
$$

A idéia inicial é observar que tais extensões (que constituem a nossa torre) são de ArtinSchreier (no sentido do capítulo 3) e, usando os resultados conhecidos em tais extensões, sistematizaremos uma forma de calcular o gênero e o número de lugares de grau $1 \mathrm{em} F_{n}$. Finalmente, tomando o limite $n \longrightarrow \infty$, atingiremos a cota de Drinfeld-Vladut.

Para uma maior praticidade na leitura, reenunciaremos a proposição 3.50. Veremos que esta será o alicerce do nosso trabalho, e que a mesma diz, dentre outras coisas, que as extensões apresentadas na definição 4.1 são do tipo Artin-Schreier.

Proposição 4.2. Seja $q$ uma potência de um primo $p$, e $F / K$ um corpo de funções algébricas sobre $K$, onde $K=\mathbb{F}_{q^{2}}$ é algebricamente fechado em $F$. Seja $\omega \in F$ e suponha que existe um lugar $P \in \mathbb{P}(F)$ tal que

$$
v_{P}(\omega)=-m, m>0, \quad e \quad m d c(m, q)=1 .
$$


Então, o polinômio $\varphi(T)=T^{q}+T-\omega \in F[T]$ é absolutamente irredutível, isto é, irredutivel em $\bar{K} \cdot F[T]$, onde $\bar{K} \cdot F$ denota o compósito do fecho algébrico de $K$ e $F$.

Seja $E=F(z)$, onde $\varphi(z)=0$, isto é, $z^{q}+z=\omega$. Então:

(i) $E / F$ é uma extensão de Galois de grau $[E: F]=q$, o grupo de Galois é abeliano elementar de expoente $p$, e $K$ é algebricamente fechado em $E$;

(ii) o lugar $P$ é totalmente ramificado em $E$, isto é, existe um único lugar $P^{\prime} \in \mathbb{P}(E)$ tal que $P^{\prime} \mid P$ e e $\left(P^{\prime} \mid P\right)=q$; temos ainda que $\operatorname{deg}\left(P^{\prime}\right)=\operatorname{deg}(P)$, e o expoente da diferente de $P^{\prime} \mid P$ é dado por:

$$
d\left(P^{\prime} \mid P\right)=(q-1)(m+1)
$$

(iii) seja $R \in \mathbb{P}(F)$, e suponhamos que $v_{R}\left(\omega-\left(u^{q}+u\right)\right) \geq 0$ para algum $u \in F$; então, $R$ é não-ramificado em $E / F$; isto ocorre em particular se $v_{R}(\omega) \geq 0$;

(iv) seja $Q \in \mathbb{P}(F)$ um zero de $\omega-\gamma$, com $\gamma \in \mathbb{F}_{q}$; entâo, a equação $\alpha^{q}+\alpha=\gamma$ tem $q$ raízes distintas $\alpha \in K$, e para cada $\alpha$ existe um único lugar $Q_{\alpha} \in \mathbb{P}(E)$ tal que $Q_{\alpha} \mid Q$ e $Q_{\alpha}$ é zero de $z-\alpha$.

\subsection{O Gênero de $F_{n} / K$}

Vamos utilizar a proposição 4.2 em cada extensão da torre construída na definição 4.1 , procedendo da seguinte forma: determinamos o divisor principal $\left(x_{n}\right)$ em $F_{n}$ (denotado por $\left.\left(x_{n}\right)^{F_{n}}\right)$, e, pela proposição 4.2 , veremos que os únicos lugares de $F_{n}$ que podem se ramificar em $F_{n+1}$ são os pólos de $x_{n}$. Então, fazendo uma classificação adequada dos lugares que estão no suporte de $\left(x_{n}\right)^{F_{n}}$, e separando-os em certos conjuntos, conseguiremos controlar as ramificações e discriminar em que conjunto estarão os lugares de $F_{n}$ que se ramificarão em $F_{n+1}$. Mostraremos ainda que tais lugares são totalmente ramificados em $F_{n+1}$, agregando outras informações, como, por exemplo, a ordem de pólo ou de zero de $x_{n}$ em cada um deles. Por outro lado, mostraremos que os demais pólos (que não são totalmente ramificados), dispostos em outros conjuntos segundo a nossa classificação, são todos não ramificados em $F_{n+1}$, o que nos permite esquecê-los no momento do cálculo do gênero de $F_{n+1}$ (ver observação 3.38). Enfim, observando que $\left[F_{n+1}: F_{n}\right]=q$, e que $K$ é algebricamente fechado em $F_{n+1}$, obtemos (usando a fórmula de Hurwitz) o gênero de $F_{n}$ em função de $n$ e de $q$.

Lema 4.3. Suponha que um lugar $P \in \mathbb{P}\left(F_{n}\right)$ é um pólo simples de $x_{n}$ em $F_{n}$, ou seja, $v_{P}\left(x_{n}\right)=-1$. Entâo, $F_{n+1} / F_{n}$ é uma extensẫo de Artin-Schreier de grau $\left[F_{n+1}: F_{n}\right]=q$ e $P$ é totalmente ramificado em $F_{n+1} / F_{n}$. Além disso, o lugar $P^{\prime} \in \mathbb{P}\left(F_{n+1}\right)$, tal que $P^{\prime} \mid P$, é um pólo simples de $x_{n+1}$.

Demonstração. Por definição, temos que $F_{n+1}=F_{n}\left(z_{n+1}\right)$, onde $z_{n+1}^{q}+z_{n+1}=x_{n}^{q+1}$. Como $P$ é pólo simples de $x_{n}$, isto é, $v_{P}\left(x_{n}\right)=-1$, temos que $v_{P}\left(x_{n}^{q+1}\right)=-(q+1)$, onde $q+1>0$ e $m d c(q+1, q)=1$. Logo, pela proposição 4.2 , temos que $\left[F_{n+1}: F_{n}\right]=q$, e que $P$ é totalmente ramificado em $F_{n+1}$. Assim, $e\left(P^{\prime} \mid P\right)=q$. 
Agora, provaremos que $v_{P^{\prime}}\left(x_{n+1}\right)=-1$. Por definição, temos que

$$
x_{n+1}=\frac{z_{n+1}}{x_{n}} .
$$

Logo, $v_{P^{\prime}}\left(x_{n+1}\right)=v_{P^{\prime}}\left(z_{n+1}\right)-v_{P^{\prime}}\left(x_{n}\right)$. Mas,

$$
\begin{aligned}
z_{n+1}^{q}+z_{n+1}=x_{n}^{q+1} & \Longrightarrow v_{P^{\prime}}\left(z_{n+1}^{q}+z_{n+1}\right)=(q+1) \cdot v_{P^{\prime}}\left(x_{n}\right) \\
& \Longrightarrow v_{P^{\prime}}\left(z_{n+1}^{q}+z_{n+1}\right)=(q+1) \cdot e\left(P^{\prime} \mid P\right) \cdot v_{P}\left(x_{n}\right) \\
& \Longrightarrow v_{P^{\prime}}\left(z_{n+1}^{q}+z_{n+1}\right)=-q \cdot(q+1) .
\end{aligned}
$$

Agora, sabemos que

$$
0>v_{P^{\prime}}\left(z_{n+1}^{q}+z_{n+1}\right) \geq \min \left\{v_{P^{\prime}}\left(z_{n+1}\right), v_{P^{\prime}}\left(z_{n+1}^{q}\right)\right\} .
$$

Logo, $v_{P^{\prime}}\left(z_{n+1}\right)<0$, e assim temos:

$$
\begin{aligned}
v_{P^{\prime}}\left(z_{n+1}^{q}\right) \neq v_{P^{\prime}}\left(z_{n+1}\right) \Longrightarrow v_{P^{\prime}}\left(z_{n+1}^{q}+z_{n+1}\right) & =\min \left\{v_{P^{\prime}}\left(z_{n+1}^{q}\right), v_{P^{\prime}}\left(z_{n+1}\right)\right\} \\
& =v_{P^{\prime}}\left(z_{n+1}^{q}\right) \\
& =q \cdot v_{P^{\prime}}\left(z_{n+1}\right) .
\end{aligned}
$$

Então:

$$
q \cdot v_{P^{\prime}}\left(z_{n+1}\right)=-q \cdot(q+1) \Longrightarrow v_{P^{\prime}}\left(z_{n+1}\right)=-(q+1)
$$

Assim,

$$
\begin{aligned}
v_{P^{\prime}}\left(x_{n+1}\right) & =v_{P^{\prime}}\left(z_{n+1}\right)-v_{P^{\prime}}\left(x_{n}\right) \\
& =-(q+1)-e\left(P^{\prime} \mid P\right) \cdot v_{P}\left(x_{n}\right) \\
& =-(q+1)-q \cdot(-1) \\
& =-1 .
\end{aligned}
$$

Lema 4.4. Para $n \geq 1$, temos que $K$ é algebricamente fechado em $F_{n}$, e o grau da extensão $F_{n} / F_{1}$ é $\left[F_{n}: F_{1}\right]=q^{n-1}$.

Demonstração. Provaremos por indução sobre $n$. Para $n=1$, temos que $F_{1}=K\left(x_{1}\right)$, onde $K$ é (obviamente) algebricamente fechado em $F_{1}$, e $\left[F_{1}: F_{1}\right]=1=q^{1-1}$. Agora, suponhamos a validade do lema para algum $n \geq 1$. Mas, $F_{n+1}=F_{n}\left(z_{n+1}\right)$, onde $z_{n+1}^{q}+$ $z_{n+1}=x_{n}^{q+1}$; temos ainda que, em $F_{1}=K\left(x_{1}\right), P_{\infty}$ é pólo simples de $x_{1}$. Logo, pelo lema $4.3, P_{\infty}$ tem uma única extensão $P_{\infty}^{(n)}$ em $F_{n}$, e este é pólo simples de $x_{n}$, isto é, $v_{P_{\infty}^{(n)}}\left(x_{n}^{q+1}\right)=-(q+1)$. Assim, pela proposição 4.2 , temos que $K$ é algebricamente fechado em $F_{n+1}$, e também que $\left[F_{n+1}: F_{n}\right]=q$. Logo:

$$
\left[F_{n+1}: F_{1}\right]=\left[F_{n+1}: F_{n}\right] \cdot\left[F_{n}: F_{1}\right]=q \cdot q^{n-1}=q^{n}=q^{(n+1)-1}
$$


O lema a seguir caracteriza um lugar específico de $F_{n}$ que tem um papel muito importante para a obtenção dos próximos resultados.

Lema 4.5. Para $n \geq 1$, existe um único lugar $Q_{n} \in \mathbb{P}\left(F_{n}\right)$ que é zero comum das funções $x_{1}, z_{2}, z_{3}, \ldots, z_{n}$. Temos ainda que $\operatorname{deg}\left(Q_{n}\right)=1$, e, para $1 \leq k \leq n$, o lugar $Q_{n}$ também é zero de $x_{k}$, e temos $v_{Q_{n}}\left(x_{k}\right)=q^{k-1}$. Na extensão $F_{n+1} / F_{n}$, o lugar $Q_{n}$ decompõe-se em $q$ lugares de $F_{n+1}$, todos de grau 1 , onde um deles é $Q_{n+1}$.

Demonstração. Para $n=1$, temos $F_{1}=K\left(x_{1}\right)$, onde $\left(x_{1}\right)^{F_{1}}=P_{0}-P_{\infty}$ é o divisor associado a $x_{1}$, e onde $P_{0}$ é o lugar de grau 1 , associado ao polinômio de grau $1(x-0) \in$ $K[x]$. Fazendo $Q_{1}=P_{0}$ temos que $Q_{1}$ é zero de $x_{1}^{q+1}-0$, e, pelo item (iv) da proposição 4.2, a equação $\alpha^{q}+\alpha=0$ tem $q$ raízes distintas em $K$, onde a cada uma delas corresponde um único lugar $Q_{\alpha}$ de $F_{2}$, que é zero de $z_{2}-\alpha$. Como $\alpha^{q}+\alpha=0$ é separável, temos que $Q_{1}$ se decompõe em $q$ outros lugares de $F_{2}$. Logo:

$$
\sum_{i=1}^{q} e_{i} f_{i}=\left[F_{2}: F_{1}\right]=q \Longrightarrow e_{i}=f_{i}=1 \quad, \quad \forall i \in\{1, \ldots, q\}
$$

Assim:

$$
\operatorname{deg}\left(Q_{1}\right)=1 \Longrightarrow \operatorname{deg}\left(Q_{\alpha}\right)=1, \forall \alpha \in K,
$$

$\operatorname{com} \alpha^{q}+\alpha=0$ (ver diagrama a seguir).

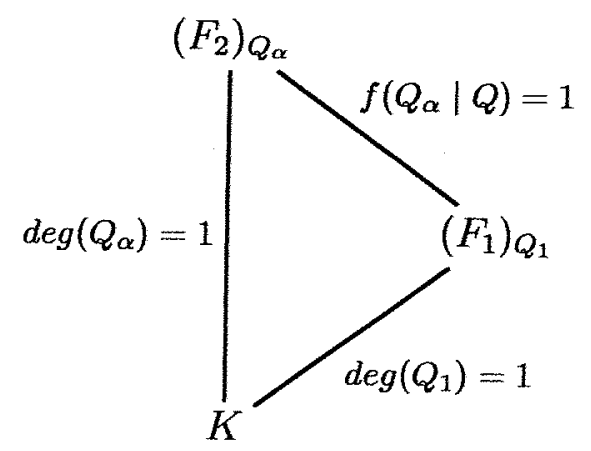

Analisemos agora o caso $n=2$. Denotemos por $Q_{2}$ o lugar de $F_{2}$, extensão de $Q_{1}$, associado à raiz $0 \in F_{q}$ de $\alpha^{q}+\alpha=0$. Já sabemos que $Q_{2}$ tem grau 1 , e $e\left(Q_{2} \mid Q_{1}\right)=1$. Logo,

$$
v_{Q_{2}}\left(x_{1}\right)=e\left(Q_{2} \mid Q_{1}\right) \cdot v_{Q_{1}}\left(x_{1}\right)=1=q^{1-1},
$$

e $Q_{2}$ é zero de $z_{2}-0=z_{2}$. Para ver que $Q_{2}$ é o único zero de $x_{1}$ e $z_{2}$ em $F_{2}$, basta observar que qualquer outro zero de $x_{1}$ em $F_{2}$ é extensão de $Q_{1}, e$, portanto,zero de $z_{2}-\alpha$, com $\alpha \neq 0$, o que é um absurdo, pois, se $v_{P}\left(z_{2}\right)>0$, então

$$
v_{P}\left(z_{2}-\alpha\right)=\min \left\{v_{P}\left(z_{2}\right), v_{P}(\alpha)\right\}=0 \text {. }
$$

Mostraremos agora que $v_{Q_{2}}\left(x_{2}\right)=q=q^{2-1}$. Observemos primeiramente que:

$$
\begin{aligned}
z_{2}^{q}+z_{2}=x_{1}^{q+1} & \Longrightarrow v_{Q_{2}}\left(z_{2}^{q}+z_{2}\right)=v_{Q_{2}}\left(x_{1}^{q+1}\right) \\
& \Longrightarrow v_{Q_{2}}\left(z_{2}^{q}+z_{2}\right)=(q+1) \cdot v_{Q_{2}}\left(x_{1}\right)=q+1 \\
& \left.\Longrightarrow v_{Q_{2}}\left(z_{2}\right)=q+1 \quad \text { (pois } Q_{2} \text { é zero de } z_{2} \text {, ou seja, } v_{Q_{2}}\left(z_{2}\right)>0\right)
\end{aligned}
$$


Logo:

$$
x_{2}=\frac{z_{2}}{x_{1}} \Longrightarrow v_{Q_{2}}\left(x_{2}\right)=v_{Q_{2}}\left(z_{2}\right)-v_{Q_{2}}\left(x_{1}\right)=q+1-1=q .
$$

Como $Q_{2}$ é zero de $x_{2}^{q+1}-0$, segue, do item (iv) da proposição 4.2 , que $Q_{2}$ se decompõe em $q$ outros lugares de $F_{3}$, cada um deles associado a uma única raiz da equação $\alpha^{q}+\alpha=0$. Naturalmente, como $Q_{2}$ tem grau 1 e se decompõe em $q=\left[F_{3}: F_{2}\right]$ outros lugares, então todos eles terão grau 1.

Enfim, suponhamos a validade do lema para algum natural $n-1$, com $n \geq 3$. Isto significa que existe um único lugar $Q_{n-1} \in \mathbb{P}\left(F_{n-1}\right)$ que é zero comum de $x_{1}, z_{2}, \ldots, z_{n-1}$, com $\operatorname{deg}\left(Q_{n-1}\right)=1$, e, para cada $1 \leq k \leq n-1$, temos $v_{Q_{n-1}}\left(x_{k}\right)=q^{k-1}$; além disso, $Q_{n-1}$ se decompõe em $q$ outros lugares de $F_{n} / F_{n-1}$, todos de grau 1 . Considere, pois, $F_{n}=F_{n-1}\left(z_{n}\right)$, onde $z_{n}^{q}+z_{n}=x_{n-1}^{q+1}$; como $Q_{n-1} \in \mathbb{P}\left(F_{n-1}\right)$ é zero de $x_{n-1}^{q+1}-0$, segue, do item (iv) da proposição 4.2 , que existe um único $Q_{n} \in \mathbb{P}\left(F_{n}\right)$, extensão de $Q_{n-1}$, tal que $Q_{n}$ é zero de $z_{n}$. Portanto, $Q_{n}$ é o único zero comum de $x_{1}, z_{2}, \ldots, z_{n}$. Então, como $Q_{n-1} \in \mathbb{P}\left(F_{n-1}\right)$ se decompõe em $q$ outros lugares de $F_{n}$, temos que

$$
\sum_{i=1}^{q} e_{i} f_{i}=\left[F_{n}: F_{n-1}\right]=q \Longrightarrow e_{i}=f_{i}=1 .
$$

Logo, todos os lugares $Q_{\alpha} \in \mathbb{P}\left(F_{n}\right)$ têm grau 1 , e, em particular, $\operatorname{deg}\left(Q_{n}\right)=1$.

Agora, para $1 \leq k \leq n-1$, temos:

$$
v_{Q_{n}}\left(x_{k}\right)=e\left(Q_{n} \mid Q_{n-1}\right) \cdot v_{Q_{n-1}}\left(x_{k}\right)=1 \cdot q^{k-1},
$$

e, portanto, $v_{Q_{n}}\left(x_{k}\right)=q^{k-1}$ para qualquer que seja $1 \leq k \leq n-1$. Para $k=n$, temos:

$$
\begin{aligned}
z_{n}^{q}+z_{n}=x_{n-1}^{q+1} & \Longrightarrow v_{Q_{n}}\left(z_{n}^{q}+z_{n}\right)=v_{Q_{n}}\left(x_{n-1}^{q+1}\right) \\
& \Longrightarrow v_{Q_{n}}\left(z_{n}^{q}+z_{n}\right)=(q+1) \cdot v_{Q_{n}}\left(x_{n-1}\right)=(q+1) \cdot q^{n-2} .
\end{aligned}
$$

Mas, como $Q_{n}$ é zero de $z_{n}$, temos

$$
v_{Q_{n}}\left(z_{n}\right)=q^{n-1}+q^{n-2}
$$

Por outro lado, temos que:

$$
\begin{aligned}
x_{n}=\frac{z_{n}}{x_{n-1}} & \Longrightarrow v_{Q_{n}}\left(x_{n}\right)=v_{Q_{n}}\left(z_{n}\right)-v_{Q_{n}}\left(x_{n-1}\right) \\
& \Longrightarrow v_{Q_{n}}\left(x_{n}\right)=q^{n-1}+q^{n-2}-q^{n-2}=q^{n-1} .
\end{aligned}
$$

Logo, concluímos que $v_{Q_{n}}\left(x_{k}\right)=q^{k-1}$, qualquer que seja $1 \leq k \leq n$.

Corolário 4.6. O lugar $Q_{n}$, conforme o lema anterior, é o único zero de $x_{n}$ e $z_{n}$ em $F_{n}$, para $n \geq 2$.

Demonstração. Assumindo que $Q_{n}$ é o único zero de $z_{n}$ em $F_{n}$, é fácil ver que $Q_{n}$ também é o único zero de $x_{n}$ em $F_{n}$, pois

$$
x_{n}=\frac{z_{n}}{x_{n-1}} \Longrightarrow\left(x_{n}\right)^{F_{n}}=\left(z_{n}\right)^{F_{n}}-\left(x_{n-1}\right)^{F_{n}}
$$


Portanto, o único zero de $z_{n}$ também é o único zero de $x_{n}$, pois

$$
z_{n}^{q}+z_{n}=x_{n-1}^{q+1} \Longrightarrow\left(v_{P}\left(z_{n}\right)<0 \Longleftrightarrow v_{P}\left(x_{n-1}\right)<0\right),
$$

ou seja, $z_{n}$ e $x_{n-1}$ têm os mesmos pólos em $F_{n}$. Mas, como $v_{P}\left(z_{n}\right)=\frac{q+1}{q} \cdot v_{P}\left(x_{n-1}\right)$ em cada um destes pólos, temos que a ordem de pólo em $z_{n}$ é ainda maior que a ordem de pólo de $x_{n-1}$, o que não permite que pólos de $x_{n-1}$ virem zeros de $x_{n}$ em $F_{n}$.

Para mostrar que $Q_{n}$ é o único zero de $z_{n}$ em $F_{n}$, procederemos por indução. Para $n=2$, tomemos $P \in \mathbb{P}\left(F_{2}\right)$ zero de $z_{2}$. Como $z_{2}^{q}+z_{2}=x_{1}^{q+1}$, temos:

$$
v_{P}\left(z_{2}^{q}+z_{2}\right)=v_{P}\left(x_{1}^{q+1}\right) \Longrightarrow v_{P}\left(z_{2}\right)=(q+1) \cdot v_{P}\left(x_{1}\right) \Longrightarrow v_{P}\left(x_{1}\right)>0 .
$$

Logo, pelo lema anterior, temos $P=Q_{2}$.

Agora, vamos admitir que $Q_{n-1}$ é o único zero de $z_{n-1}$ em $F_{n-1}$. Usando a notação do lema 4.5 , tomemos $Q_{n} \in \mathbb{P}\left(F_{n}\right)$. Temos que $Q_{n}$ é zero de $z_{n}$. Se existir outro $Q \in \mathbb{P}\left(F_{n}\right)$ que também seja zero de $z_{n}$, então, como $z_{n}^{q}+z_{n}=x_{n-1}^{q+1}$, temos que $Q$ é zero de $x_{n-1}$ em $F_{n}$, ou seja, $Q$ é extensão de um zero de $x_{n-1}$ em $F_{n-1}$; portanto, $Q \mid Q_{n-1}$. Logo, $Q$ é zero de $z-\alpha$, onde $\alpha \in K \backslash\{0\}$ e $\alpha^{q}+\alpha=0$, o que é absurdo, pois

$$
v_{Q}\left(z_{n}\right)>0 \Longrightarrow v_{Q}\left(z_{n}-\alpha\right)=0 \text {. }
$$

Corolário 4.7. Para $n \geq 2$, o suporte de $\left(x_{n}\right)$ em $F_{n}$ é formado pelas extensões dos lugares do suporte de $\left(x_{n-1}\right)$ em $F_{n-1}$.

Demonstração. É evidente a partir da demonstração do corolário anterior.

Conforme mencionamos anteriormente, dividiremos o suporte de $\left(x_{n}\right)^{F_{n}}$ em determinados conjuntos de lugares de modo a facilitar o controle das ramificações. Considerando o corolário 4.7, tomaremos, para $n \geq 2 \mathrm{em} F_{n}$, as extensões dos lugares do suporte de $\left(x_{1}\right)^{F_{1}}=Q_{1}-P_{\infty}$, e as dividiremos em certos conjuntos segundo um critério que será justificado à posteriori. A definição seguinte formaliza toda esta idéia.

Definição 4.8. (Veja figura $\mathbf{\Lambda}$ )

(i) Para $n \geq 2$, seja

$$
S_{0}^{(n)}:=\left\{P \in \mathbb{P}\left(F_{n}\right) \mid P \cap F_{n-1}=Q_{n-1} \text { e } P \neq Q_{n}\right\} .
$$

(ii) Para $1 \leq i \leq\left[\frac{n-3}{2}\right]$, com $n \geq 5$, seja

$$
S_{i}^{(n)}:=\left\{P \in \mathbb{P}\left(F_{n}\right) \mid P \cap F_{n-1} \in S_{i-1}^{(n-1)}\right\} .
$$

(iii) Seja $P_{\infty} \in \mathbb{P}\left(F_{1}\right)$ o pólo de $x_{1}$ em $F_{1}$. Definimos

$$
S^{(1)}=\left\{P_{\infty}\right\} \quad \text { e } S^{(2)}=\left\{P \in \mathbb{P}\left(F_{2}\right) \mid P \in S_{0}^{(2)} \text { ou } P \cap F_{1}=P_{\infty}\right\},
$$

ou seja, $S^{(2)}$ contém todos os lugares de $F_{2}$ que são pólos de $x_{1}$, ou zero de $x_{1} e$ $z_{2}-\alpha, \operatorname{com} \alpha \in K \backslash\{0\}$ e $\alpha^{q}+\alpha=0$. 
(iv) Para $n \geq 3$ impar, definimos

$$
S^{(n)}:=\left\{P \in \mathbb{P}\left(F_{n}\right) \mid P \cap F_{n-1} \in S^{(n-1)}\right\},
$$

e, para $n \geq 4$ par, definimos

$$
S^{(n)}:=\left\{P \in \mathbb{P}\left(F_{n}\right) \mid P \cap F_{n-1} \in S^{(n-1)} \cup S_{\frac{n-4}{2}}^{(n-1)}\right\}
$$

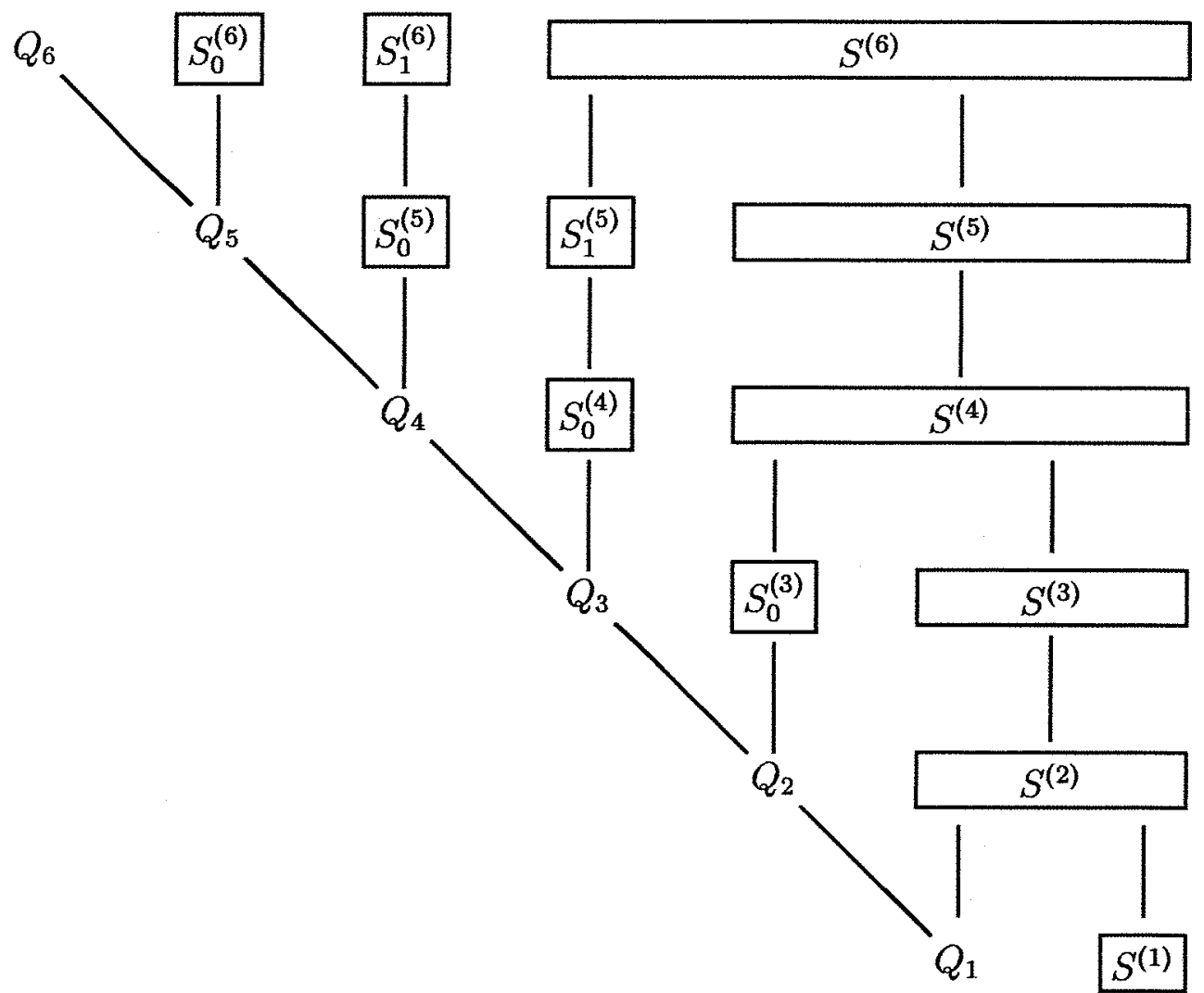

Figura

Observação 4.9. De agora em diante, a menos de menção em contrário, nos reportaremos ao $S_{i}^{(n)}$ subentendendo $n \geq 3$. Assim, pelo corolário 4.7 e pela definição 4.8 , podemos observar que o suporte de $\left(x_{n}\right)$ em $F_{n}$, para $n \geq 3$, é dado pelo conjunto $\left\{Q_{n}\right\} \cup X \cup S^{(n)}$, onde

$$
X=\bigcup_{i=0}^{\left[\frac{n-3}{2}\right]} S_{i}^{(n)}
$$


Passaremos agora à parte mais técnica do trabalho, que é mostrar que os lugares de $S_{i}^{(n)}$ são não-ramificados em $F_{n+1}$. O principal objetivo desta próxima etapa é mostrar que, dado $P \in S_{i}^{(n)}$, existe $u \in F_{n}$ tal que $x_{n}^{q+1}=\wp(u)+\mathcal{O}(1)$ em $P$; daí, pelo item (iii) da proposição 4.2 , concluímos que $P$ é não-ramificado em $F_{n+1}$.

A maioria dos resultados a seguir será mais facilmente interpretada com o auxílio da figura $\boldsymbol{\phi}$.

Para simplificar as próximas demonstrações, apresentaremos o seguinte lema:

Lema 4.10. (i) Para $2 \leq k \leq n$, se $P \in \mathbb{P}\left(F_{n}\right)$ está acima de $Q_{k}$ (isto é, $P$ é zero de $\left.x_{1}, z_{2}, \ldots, z_{k}\right)$, entâo:

$$
\frac{z_{k}^{q^{2}}}{x_{k-1}^{q+1}}=\mathcal{O}\left(x_{k-1}^{(q+1)\left(q^{2}-1\right)}\right) \text { em } P
$$

(ii) Se $P \in \mathbb{P}\left(F_{n}\right)$ é zero de $x_{1}, z_{2}, \ldots, z_{n-1}$ e $z_{n}-\alpha$, onde $\alpha \in K \backslash\{0\}$ e $\alpha^{q}+\alpha=0$, então

$$
v_{P}\left(\frac{z_{n}-\alpha}{x_{n-1}^{q+1}}\right)=0
$$

Em particular,

$$
\frac{z_{n}-\alpha}{x_{n-1}^{q+1}}=\mathcal{O}(1)
$$

Demonstração. (i) Com efeito, temos que:

$$
\frac{z_{k}^{q^{2}}}{x_{k-1}^{q+1}}=x_{k-1}^{(q+1)\left(q^{2}-1\right)} \cdot\left(\frac{z_{k}}{x_{k-1}^{q+1}}\right)^{q^{2}}
$$

Como $v_{P}\left(z_{k}\right)>0$ e $z_{k}^{q}+z_{k}=x_{k-1}^{q+1}$, segue que:

$$
\begin{aligned}
v_{P}\left(z_{k}^{q}+z_{k}\right)=v_{P}\left(x_{k-1}^{q+1}\right) & \Longrightarrow v_{P}\left(z_{k}\right)=v_{P}\left(x_{k-1}^{q+1}\right) \\
& \Longrightarrow v_{P}\left(\frac{z_{k}}{x_{k-1}^{q+1}}\right)=0
\end{aligned}
$$

Logo:

$$
\frac{z_{k}^{q^{2}}}{x_{k-1}^{q+1}}=\mathcal{O}\left(x_{k-1}^{(q+1)\left(q^{2}-1\right)}\right) .
$$

(ii) De fato, como $z_{n}^{q}+z_{n}=x_{n-1}^{q+1}$ e $\alpha^{q}+\alpha=0$, temos que $\left(z_{n}-\alpha\right)^{q}+\left(z_{n}-\alpha\right)=x_{n-1}^{q+1}$.

Dado que $v_{P}\left(z_{n}-\alpha\right)>0$, temos

$$
v_{P}\left(z_{n}-\alpha\right)=v_{P}\left(x_{n-1}^{q+1}\right) \Longrightarrow v_{P}\left(\frac{z_{n}-\alpha}{x_{n-1}^{q+1}}\right)=0
$$


Proposição 4.11. Seja $0 \leq i \leq\left[\frac{n-3}{2}\right]$ e $P \in S_{i}^{(n)}$. Então o lugar $P$ é não-ramificado na extensão $F_{n+1} / F_{n}$.

Para provar esta proposição, precisaremos de mais dois lemas. Lembramos ainda que em algumas passagens das próximas demonstrações serão utilizadas as propriedades $P_{i}$, apresentadas na seção 3.8 .

Lema 4.12. Seja $2 \leq k \leq n$ e $P \in \mathbb{P}\left(F_{n}\right)$ um lugar acima de $Q_{k}$ (isto é, $P$ é zero de $\left.x_{1}, z_{2}, \ldots, z_{k}\right)$. Então, no lugar $P$, temos:

$$
\text { (I) } \quad x_{k}=x_{k-1}^{q}\left(1-x_{k-1}^{(q+1)(q-1)}+\mathcal{O}\left(x_{k-1}^{(q+1)\left(q^{2}-1\right)}\right)\right)
$$

$e$

$$
\text { (II) } \quad x_{k}^{-(q+1)}=\wp\left(x_{k-1}^{-(q+1)}\right)+\mathcal{O}(1) .
$$

Demonstração. Consideremos a equação $z_{k}^{q}+z_{k}=x_{k-1}^{q+1}$. Como $z_{k}=x_{k-1}^{q+1}-z_{k}^{q}$, temos

$$
\begin{aligned}
z_{k}=x_{k-1}^{q+1}-\left(x_{k-1}^{q+1}-z_{k}^{q}\right)^{q} & \Longrightarrow z_{k}=x_{k-1}^{q+1}-x_{k-1}^{q(q+1)}+z_{k}^{q^{2}} \\
& \Longrightarrow z_{k}=x_{k-1}^{q+1} \cdot\left(1-x_{k-1}^{q^{2}-1}+\frac{z_{k}^{q^{2}}}{x_{k-1}^{q+1}}\right) \\
& \Longrightarrow z_{k}=x_{k-1}^{q+1} \cdot\left(1-x_{k-1}^{q^{2}-1}+\mathcal{O}\left(x_{k-1}^{(q+1)\left(q^{2}-1\right)}\right)\right) \text { em } P \text { (lema 4.10(i)) } \\
& \Longrightarrow x_{k}=\frac{z_{k}}{x_{k-1}}=x_{k-1}^{q} \cdot\left(1-x_{k-1}^{q^{2}-1}+\mathcal{O}\left(x_{k-1}^{(q+1)\left(q^{2}-1\right)}\right)\right) \text { em } P .
\end{aligned}
$$

Obtemos, assim, a parte (I) do lema.

Agora, fazendo $y:=x_{k-1}^{q+1}$, temos

$$
\begin{aligned}
x_{k}=x_{k-1}^{q} \cdot\left(1-y^{q-1}+\mathcal{O}\left(y^{q^{2}-1}\right)\right) & \stackrel{P_{5}}{\Longrightarrow} x_{k}=x_{k-1}^{q} \cdot\left(1-y^{q-1}+\mathcal{O}\left(y^{q}\right)\right) \\
& \Longrightarrow x_{k}^{-1}=x_{k-1}^{-q} \cdot\left(1-y^{q-1}+\mathcal{O}\left(y^{q}\right)\right)^{-1} \\
& \Longrightarrow x_{k}^{-1}=x_{k-1}^{-q} \cdot\left(1+y^{q-1}+\mathcal{O}\left(y^{q}\right)\right) \\
& \Longrightarrow x_{k}^{-(q+1)}=x_{k-1}^{-q(q+1)} \cdot\left(1+y^{q-1}+\mathcal{O}\left(y^{q}\right)\right)^{q+1} \\
& \stackrel{P_{7}}{\Longrightarrow} x_{k}^{-(q+1)}=y^{-q} \cdot\left(1+y^{q-1}+\mathcal{O}\left(y^{q}\right)\right) \\
& \stackrel{P_{0}}{\Longrightarrow} x_{k}^{-(q+1)}=y^{-q}+y^{-1}+\mathcal{O}(1) \\
& \Longrightarrow x_{k}^{-(q+1)}=\wp\left(y^{-1}\right)+\mathcal{O}(1)
\end{aligned}
$$

de onde segue (II).

Lema 4.13. Seja $0 \leq i \leq\left[\frac{n-3}{2}\right]$. Então, temos em $P$ :

$$
x_{n}^{q+1}=\gamma \cdot x_{n-2 i-1}^{-(q+1)}+\mathcal{O}(1)
$$

para algum $\gamma \in \mathbb{F}_{q} \backslash\{0\}$. 
Demonstração. Provaremos por indução sobre $i$. Para o caso $i=0$, tomamos $P \in S_{0}^{(n)}$, ou seja $P \mid Q_{n-1}$ e $P \neq Q_{n}$; então, $P$ é zero comum de $x_{1}, z_{2}, \ldots, z_{n-1}$ e $z_{n}-\alpha$, onde $\alpha \in K \backslash\{0\}$ é tal que $\alpha^{q}+\alpha=0$.

Assim, temos que:

$$
\begin{aligned}
z_{n}^{q}+z_{n}=x_{n-1}^{q+1} & \Longrightarrow\left(z_{n}-\alpha\right)^{q}+\left(z_{n}-\alpha\right)=x_{n-1}^{q+1} \\
& \Longrightarrow z_{n}-\alpha=x_{n-1}^{q+1}-\left(z_{n}-\alpha\right)^{q} \\
& \Longrightarrow z_{n}-\alpha=x_{n-1}^{q+1}+x_{n-1}^{q(q+1)} \cdot\left[-\frac{z_{n}-\alpha}{x_{n-1}^{q+1}}\right]^{q} \\
& \Longrightarrow z_{n}-\alpha=x_{n-1}^{q+1}+\mathcal{O}\left(x_{n-1}^{q(q+1)}\right) \quad(\text { lema } 4.10(\mathrm{ii}))
\end{aligned}
$$

Logo:

$$
\begin{aligned}
x_{n}^{q+1} \cdot x_{n-1}^{q+1} & =z_{n}^{q+1}=\left(\left(z_{n}-\alpha\right)+\alpha\right)^{q+1} \\
& =\left(\left(z_{n}-\alpha\right)^{q}+\alpha^{q}\right) \cdot\left(\left(z_{n}-\alpha\right)+\alpha\right) \\
& =\alpha^{q+1}+\alpha^{q} \cdot\left(z_{n}-\alpha\right)+\alpha \cdot\left(z_{n}-\alpha\right)^{q}+\left(z_{n}-\alpha\right)^{q+1} .
\end{aligned}
$$

Fazendo a substituição, temos:

$x_{n}^{q+1} \cdot x_{n-1}^{q+1}=\alpha^{q+1}+\alpha^{q} \cdot\left(x_{n-1}^{q+1}+\mathcal{O}\left(x_{n-1}^{q(q+1)}\right)\right)+\alpha \cdot\left(x_{n-1}^{q(q+1)}+\mathcal{O}\left(x_{n-1}^{q^{2}(q+1)}\right)\right)+\left(x_{n-1}^{q+1}+\mathcal{O}\left(x_{n-1}^{q(q+1)}\right)\right)^{q+1}$, o que, por $P_{3}$ e $P_{4}$, implica

$$
\begin{aligned}
x_{n}^{q+1} \cdot x_{n-1}^{q+1}=\alpha^{q+1}+\alpha^{q} \cdot x_{n-1}^{q+1}+\mathcal{O}\left(x_{n-1}^{q(q+1)}\right) & \Longrightarrow \underbrace{x_{n}^{q+1}=\frac{\alpha^{q+1}}{x_{n-1}^{q+1}}+\alpha^{q}+\mathcal{O}\left(x_{n-1}^{q^{2}-1}\right)}_{(*)} \\
& \Longrightarrow x_{n}^{q+1}=\frac{\alpha^{q+1}}{x_{n-1}^{q+1}}+\mathcal{O}(1) .
\end{aligned}
$$

Como $\alpha \in K$, temos que $\gamma:=\alpha^{q+1} \in \mathbb{F}_{q}$, pois $\left(\alpha^{q+1}\right)^{q}=\alpha^{q^{2}+q}=\alpha^{q+1}$. Deste modo, podemos concluir que $x_{n}^{q+1}=\gamma \cdot x_{n-1}^{-(q+1)}+\mathcal{O}(1)$ em $P \in S_{0}^{(n)}$, qualquer que seja $n \geq 3$.

Agora, tomamos $i \geq 1$ e supomos que o lema seja válido para $i-1$. Dado $P \in S_{i}^{(n)}$, temos (pela definição do $S_{i}^{(n)}$ ) que $P \mid Q_{n-i-1}$, e, como $P \cap F_{n-1} \in S_{i-1}^{(n-1)}$, segue (da hipótese) que:

$$
\begin{aligned}
x_{n-1}^{q+1} & =\gamma \cdot x_{(n-1)-2(i-1)-1}^{-(q+1)}+\mathcal{O}(1) \\
\Longrightarrow \quad x_{n-1}^{q+1} & =\gamma \cdot x_{n-2 i}^{-(q+1)}+\mathcal{O}(1), \gamma \in \mathbb{F}_{q} \backslash\{0\}
\end{aligned}
$$

Como $P$ está sobre $Q_{n-i-1}$, temos que $P$ também está sobre $Q_{n-2 i}$, pois $n-2 i \leq n-i-1$ para todo $i \geq 1$. Por outro lado, como $i \leq \frac{n-3}{2}$, temos que $n-2 i \geq 3$. Logo, pelo item (II) do lema 4.12, obtemos:

$$
x_{n-2 i}^{-(q+1)}=\wp\left(x_{n-2 i-1}^{-(q+1)}\right)+\mathcal{O}(1) \text { em } P .
$$


Então, usando $P_{0}$, temos:

$$
\gamma \cdot x_{n-2 i}^{-(q+1)}=\gamma \cdot \wp\left(x_{n-2 i-1}^{-(q+1)}\right)+\mathcal{O}(\gamma)
$$

o que (por $P_{1}$ ) implica:

$$
\gamma \cdot x_{n-2 i}^{-(q+1)}+\mathcal{O}(1)=\wp\left(\gamma \cdot x_{n-2 i-1}^{-(q+1)}\right)+\mathcal{O}(\gamma)+\mathcal{O}(1) \stackrel{P_{3},(1)}{\Longrightarrow} x_{n-1}^{q+1}=\wp\left(\gamma \cdot x_{n-2 i-1}^{-(q+1)}\right)+\mathcal{O}(1) .
$$

Mas, como $z_{n}^{q}+z_{n}=x_{n-1}^{q+1}$, temos que:

$$
\begin{aligned}
\wp\left(z_{n}\right)=\wp\left(\gamma \cdot x_{n-2 i-1}^{-(q+1)}\right)+\mathcal{O}(1) & \Longrightarrow \wp\left(z_{n}\right)-\wp\left(\gamma \cdot x_{n-2 i-1}^{-(q+1)}\right)=\mathcal{O}(1) \\
& \Longrightarrow \wp\left(z_{n}-\gamma \cdot x_{n-2 i-1}^{-(q+1)}\right)=\mathcal{O}(1) \\
& \Longrightarrow z_{n}-\gamma \cdot x_{n-2 i-1}^{-(q+1)}=\mathcal{O}(1) \\
& \Longrightarrow z_{n}=\gamma \cdot x_{n-2 i-1}^{-(q+1)}+\mathcal{O}(1)
\end{aligned}
$$

Como $x_{n}=z_{n} / x_{n-1}$, obtemos então:

$$
x_{n}^{q+1}=\frac{\left(z_{n} \cdot x_{n-2 i}\right)^{q+1}}{\left(x_{n-1} \cdot x_{n-2 i}\right)^{q+1}}=A^{q+1} \cdot B^{-1}
$$

onde $A:=z_{n} \cdot x_{n-2 i}$ e $B:=x_{n-1}^{q+1} \cdot x_{n-2 i}^{q+1}$.

Como, em particular, $P$ está sobre $Q_{n-2 i}$, segue (do item (I) do lema 4.12) que:

$$
\begin{aligned}
x_{n-2 i} & =x_{n-2 i-1}^{q} \cdot\left(1-x_{n-2 i-1}^{(q+1)(q-1)}+x_{n-2 i-1}^{(q+1)\left(q^{2}-1\right)} \cdot t\right), t \in O_{P} \\
\Longrightarrow \quad x_{n-2 i} & =x_{n-2 i-1} \cdot[\underbrace{x_{n-2 i-1}^{q-1} \cdot\left(1-x_{n-2 i-1}^{(q+1)(q-1)}+x_{n-2 i-1}^{(q+1)\left(q^{2}-1\right)} \cdot t\right)}_{(*)}]
\end{aligned}
$$

Entretanto, $P$ também está acima de $Q_{n-2 i-1}$. Logo, $v_{P}\left(x_{n-2 i-1}\right)>0$ e, conseqüentemente, $(*)$ está em $O_{P}$. Portanto, $x_{n-2 i}=\mathcal{O}\left(x_{n-2 i-1}\right)$.

Agora, como $B=x_{n-1}^{q+1} \cdot x_{n-2 i}^{q+1}$, temos, de (1), que:

$$
\begin{aligned}
B=\left(\gamma \cdot x_{n-2 i}^{-(q+1)}+\mathcal{O}(1)\right) \cdot x_{n-2 i}^{q+1} & \Longrightarrow B=\gamma+\mathcal{O}\left(x_{n-2 i}^{q+1}\right) \\
& \Longrightarrow B^{-1}=\gamma^{-1}+\mathcal{O}\left(x_{n-2 i}^{q+1}\right) \\
& \Longrightarrow B^{-1}=\gamma^{-1}+\mathcal{O}\left(x_{n-2 i-1}^{q+1}\right)
\end{aligned}
$$

Por outro lado, sabemos que $A=z_{n} \cdot x_{n-2 i}$. Mas, por (2), temos:

$$
z_{n}=\gamma \cdot x_{n-2 i-1}^{-(q+1)}+\mathcal{O}(1)
$$

enquanto que, pelo item (I) do lema 4.12, temos

$$
x_{n-2 i}=x_{n-2 i-1}^{q} \cdot\left(1-x_{n-2 i-1}^{q^{2}-1}+\mathcal{O}\left(x_{n-2 i-1}^{(q+1)\left(q^{2}-1\right)}\right)\right),
$$

e, usando $P_{4}$, obtemos:

$$
x_{n-2 i}=x_{n-2 i-1}^{q} \cdot\left(1+\mathcal{O}\left(x_{n-2 i-1}^{q^{2}-1}\right)\right)
$$


Logo:

$$
\begin{aligned}
A & =\left(\gamma \cdot x_{n-2 i-1}^{-(q+1)}+\mathcal{O}(1)\right) \cdot x_{n-2 i-1}^{q} \cdot\left(1+\mathcal{O}\left(x_{n-2 i-1}^{q^{2}-1}\right)\right) \\
& =\left(\gamma \cdot x_{n-2 i-1}^{-1}+\mathcal{O}\left(x_{n-2 i-1}^{q}\right)\right) \cdot\left(1+\mathcal{O}\left(x_{n-2 i-1}^{q^{2}-1}\right)\right) \\
& =\gamma \cdot x_{n-2 i-1}^{-1}+t_{2} \cdot x_{n-2 i-1}^{q}+t_{1} \cdot \gamma \cdot x_{n-2 i-1}^{q^{2}-2}+t_{1} \cdot t_{2} \cdot x_{n-2 i-1}^{q^{2}+q-1}
\end{aligned}
$$

onde $t_{1}, t_{2} \in O_{P}$. Logo:

$$
A=\gamma \cdot x_{n-2 i-1}^{-1}+x_{n-2 i-1}^{q} \cdot \underbrace{\left(t_{2}+t_{1} \cdot \gamma \cdot x_{n-2 i-1}^{q^{2}-q-2}+t_{1} \cdot t_{2} \cdot x_{n-2 i-1}^{q^{2}-1}\right)}_{(* *)}
$$

Como (**) está em $O_{P}$, temos que:

$$
\begin{aligned}
& A=\gamma \cdot x_{n-2 i-1}^{-1}+\mathcal{O}\left(x_{n-2 i-1}^{q}\right) \\
\Longrightarrow & A^{q+1}=\left(\gamma^{q} \cdot x_{n-2 i-1}^{-q}+t^{q} \cdot x_{n-2 i-1}^{q^{2}}\right) \cdot\left(\gamma \cdot x_{n-2 i-1}^{-1}+t \cdot x_{n-2 i-1}^{q}\right), \text { onde } t \in O_{P} \\
\Longrightarrow & A^{q+1}=\gamma^{q+1} \cdot x_{n-2 i-1}^{-(q+1)}+(\underbrace{\gamma \cdot t^{q} \cdot x_{n-2 i-1}^{q^{2}-1}+t^{q+1} \cdot x_{n-2 i-1}^{q^{2}+q}+t \cdot \gamma^{q}}_{\in O_{P}}) \\
\Longrightarrow & A^{q+1}=\gamma^{q+1} \cdot x_{n-2 i-1}^{-(q+1)}+\mathcal{O}(1)=\gamma^{2} \cdot x_{n-2 i-1}^{-(q+1)}+\mathcal{O}(1), \text { pois } \gamma \in \mathbb{F}_{q} .
\end{aligned}
$$

Assim, fazendo as substituições em (3), obtemos:

$$
\begin{aligned}
& x_{n}^{q+1}=A^{q+1} \cdot B^{-1}=\left(\gamma^{2} \cdot x_{n-2 i-1}^{-(q+1)}+\mathcal{O}(1)\right) \cdot\left(\gamma^{-1}+\mathcal{O}\left(x_{n-2 i-1}^{q+1}\right)\right) \\
\Longrightarrow & x_{n}^{q+1}=\gamma \cdot x_{n-2 i-1}^{-(q+1)}+\mathcal{O}\left(\gamma^{-1}\right)+\mathcal{O}\left(\gamma^{2}\right)+\mathcal{O}\left(x_{n-2 i-1}^{q+1}\right) \\
\Longrightarrow & x_{n}^{q+1}=\gamma \cdot x_{n-2 i-1}^{-(q+1)}+\mathcal{O}(1) .
\end{aligned}
$$

Finalmente, podemos demonstrar a proposição 4.11 .

Demonstração da proposição 4.11. Dado $P \in S_{i}^{(n)}$, onde $0 \leq i \leq\left[\frac{n-3}{2}\right]$, temos (do lema 4.13) que, em $P$, vale:

$$
x_{n}^{q+1}=\gamma \cdot x_{n-2 i-1}^{-(q+1)}+\mathcal{O}(1), \operatorname{com} \gamma \in \mathbb{F}_{q} .
$$

Como $P$ está sobre $Q_{n-i-1}$ e $2 \leq n-2 i-1 \leq n-i-1$, segue (do item (II) do lema 4.12) que:

$$
\begin{aligned}
x_{n-2 i-1}^{-(q+1)}=\wp\left(x_{n-2 i-2}^{-(q+1)}\right)+\mathcal{O}(1) & \stackrel{P_{0}, P_{1}}{\Longrightarrow} \gamma \cdot x_{n-2 i-1}^{-(q+1)}+\mathcal{O}(1)=\wp\left(\gamma \cdot x_{n-2 i-2}^{-(q+1)}\right)+\mathcal{O}(\gamma)+\mathcal{O}(1) \\
& \Longrightarrow x_{n}^{q+1}=\wp\left(\gamma \cdot x_{n-2 i-2}^{-(q+1)}\right)+\mathcal{O}(1) .
\end{aligned}
$$

Como $F_{n+1}=F_{n}\left(z_{n+1}\right)$, onde $z_{n+1}^{q}+z_{n+1}=x_{n}^{q+1}$, concluímos, do item (iii) da proposição 4.2 , que $P$ é não-ramificado em $F_{n+1}$. 
Pelo corolário 4.6, já sabemos que $Q_{n}$ é o único zero de $x_{n}$ em $F_{n}$, e ainda que $v_{Q_{n}}\left(x_{n}\right)=$ $q^{n-1}$ (lema 4.5). Para obter o divisor $\left(x_{n}\right)$ em $F_{n}$ é necessário determinar a ordem de pólo, em $x_{n}$, dos lugares de $S_{i}^{(n)}$ e $S^{(n)}$. Nesta direção, temos o seguinte lema:

Lema 4.14. (i) Seja $P \in S_{i}^{(n)}$, com $0 \leq i \leq\left[\frac{n-3}{2}\right]$. Então $v_{P}\left(x_{n}\right)=-q^{n-2 i-2}$.

(ii) $P \in S^{(n)} \Longrightarrow v_{P}\left(x_{n}\right)=-1$, e, portanto, $P$ é totalmente ramificado em $F_{n+1}$.

Demonstração. (i) Se $P \in S_{i}^{(n)}$, então $P$ está sobre $Q_{n-i-1}$, ou seja, $P$ está no topo de uma "torre de lugares" $P_{j} \in S_{i-j}^{(n-j)}$, onde $j=1, \ldots, i$, os quais (pela proposição 4.11) são todos não-ramificados. Logo $e\left(P \mid Q_{n-i-1}\right)=1$.

Por outro lado, também sabemos que $e\left(Q_{n-i-1} \mid Q_{n-2 i-1}\right)=1$. Logo: $v_{P}\left(x_{n-2 i-1}\right)=e\left(P \mid Q_{n-2 i-1}\right) \cdot v_{Q_{n-2 i-1}}\left(x_{n-2 i-1}\right) \Longrightarrow v_{P}\left(x_{n-2 i-1}\right)=1 \cdot v_{Q_{n-2 i-1}}\left(x_{n-2 i-1}\right)$. Assim, pelo lema 4.5, temos que:

$$
v_{P}\left(x_{n-2 i-1}\right)=q^{n-2 i-2} .
$$

Mas, do lema 4.13, segue que:

Portanto:

$$
x_{n}^{q+1}=\gamma \cdot x_{n-2 i-1}^{-(q+1)}+\mathcal{O}(1) \text { em } P \in S_{i}^{(n)} .
$$

Então:

$$
v_{P}\left(x_{n}^{q+1}\right)=v_{P}\left(\gamma \cdot x_{n-2 i-1}^{-(q+1)}+t\right), \text { onde } v_{P}(t) \geq 0
$$

$$
\begin{aligned}
(q+1) \cdot v_{P}\left(x_{n}\right) & =\min \left\{v_{P}\left(x_{n-2 i-1}^{-(q+1)}\right), v_{P}(t)\right\} \\
& =\min \left\{-(q+1) \cdot q^{n-2 i-2}, v_{P}(t)\right\} \\
& =-(q+1) \cdot q^{n-2 i-2} .
\end{aligned}
$$

Logo, $v_{P}\left(x_{n}\right)=-q^{n-2 i-2}$.

(ii) Procederemos por indução sobre $n$.

- Caso $n=1$ :

Imediato, pois

$$
v_{p_{\infty}}\left(x_{1}\right)=-1
$$

- Caso $n=2$ :

Se $P \cap F_{1}=P_{\infty}$, então, pelo lema 4.3, temos que $v_{P}\left(x_{2}\right)=-1$. Por outro lado, se $P \in S_{0}^{(2)}$, então $P \cap F_{1}=Q_{1}$ e $P \neq Q_{2}$. Logo, $v_{P}\left(z_{2}-\alpha\right)>0$ para algum $\alpha \in K \backslash\{0\}, \operatorname{com} \alpha^{q}+\alpha=0$, e, portanto, $v_{P}\left(z_{2}\right)=0$. Assim, temos que:

$$
\begin{aligned}
x_{2}=\frac{z_{2}}{x_{1}} & \Longrightarrow v_{P}\left(x_{2}\right)=v_{P}\left(z_{2}\right)-v_{P}\left(x_{1}\right) \\
& \Longrightarrow v_{P}\left(x_{2}\right)=-e\left(P \mid Q_{1}\right) \cdot v_{Q_{1}}\left(x_{1}\right)=-1 .
\end{aligned}
$$


- Caso $n \geq 3$ :

- Caso 1: $n$ é impar.

Se $P \in S^{(n)}$, tomamos $P^{\prime}=P \cap F_{n-1} \in S^{(n-1)}$, e, por hipótese, $v_{P^{\prime}}\left(x_{n-1}\right)=$ -1 . Logo, pelo lema 4.3, $P$ é pólo simples de $x_{n}$.

- Caso 2: $n$ é par (e, portanto, $n \geq 4$ ).

- Se $P^{\prime}=P \cap F_{n-1} \in S^{(n-1)}$, repetimos o argumento do caso 1 .

- Se $P^{\prime}=P \cap F_{n-1} \in S_{\frac{n-4}{2}}^{(n-1)}$, então, pelo item (i) acima, temos $v_{P^{\prime}}\left(x_{n-1}\right)=$ $-q$. Como $z_{n}^{q}+z_{n}=x_{n-1}^{q+1}$ e $P^{\prime}$ é não-ramificado em $F_{n}$, segue que:

$$
\begin{aligned}
v_{P}\left(z_{n}^{q}+z_{n}\right)=v_{P}\left(x_{n-1}^{q+1}\right) & \Longrightarrow v_{P}\left(z_{n}^{q}+z_{n}\right)=e\left(P \mid P^{\prime}\right) \cdot v_{P^{\prime}}\left(x_{n-1}^{q+1}\right) \\
& \Longrightarrow v_{P}\left(z_{n}^{q}+z_{n}\right)=1 \cdot(q+1) \cdot v_{P^{\prime}}\left(x_{n-1}\right) \\
& \Longrightarrow v_{P}\left(z_{n}^{q}+z_{n}\right)=-q \cdot(q+1) \\
& \Longrightarrow v_{P}\left(z_{n}\right)=-(q+1)
\end{aligned}
$$

Mas, $x_{n}=z_{n} / x_{n-1} ;$ portanto,

$$
v_{P}\left(x_{n}\right)=v_{P}\left(z_{n}\right)-v_{P}\left(x_{n-1}\right) \Longrightarrow v_{P}\left(x_{n}\right)=-(q+1)-(-q)=-1 .
$$

Logo, como $v_{P}\left(x_{n}\right)=-1$, pelo lema 4.3 temos que $P$ é totalmente ramificado em $F_{n+1}$.

Lema 4.15 .

(a) $\quad\left(x_{n}\right)^{F_{n}}=q^{n-1} \cdot Q_{n}-\left(\sum_{i=0}^{\left[\frac{n-3}{2}\right]} q^{n-2 i-2} \cdot D_{i}^{(n)}\right)-D^{(n)}$, onde $\quad D_{i}^{(n)}:=\sum_{P \in S_{i}^{(n)}} P \quad$ e $\quad D^{(n)}:=\sum_{P \in S^{(n)}} P$.

(b) $\quad \operatorname{deg} D_{i}^{(n)}=q^{i} \cdot(q-1) \quad e \quad \operatorname{deg} D^{(n)}=q^{\left[\frac{n}{2}\right]}$.

\section{Demonstração.}

(a) Pela observação 4.9 , sabemos que o suporte de $\left(x_{n}\right)^{F_{n}}$ é formado pelos lugares de $S_{i}^{(n)}, S^{(n)}$ e por $Q_{n}$. Usando o fato que $v_{Q_{n}}\left(x_{n}\right)=q^{n-1}$ (lema 4.5$)$ e o lema 4.14 , segue o item (a).

(b)

- Primeiramente, mostremos que $\operatorname{deg}\left(D_{i}^{(n)}\right)=q^{i} \cdot(q-1)$. Procederemos por indução sobre $i$. 
- Para $i=0$, temos que $S_{0}^{(n)}$ é formado pelos $q-1$ lugares de grau 1 , extensões de $Q_{n-1}$, e que estão associados às raízes de $\alpha^{q-1}=-1, \alpha \in K$. Logo, $\operatorname{deg}\left(D_{0}^{(n)}\right)=q-1=q^{0} \cdot(q-1)$.

- Para $1 \leq i \leq\left[\frac{n-3}{2}\right]$, temos que $S_{i}^{(n)}$ é formado pelas extensões dos lugares de $S_{i-1}^{(n-1)}$ que, por serem não-ramificados em $F_{n}$, são tais que

$$
D_{i}^{(n)}=\sum_{P \in S_{i-1}^{(n-1)}} \sum_{P^{\prime} \mid P}(\underbrace{e\left(P^{\prime} \mid P\right)}_{1} \cdot P^{\prime}) \Longrightarrow D_{i}^{(n)}=\operatorname{Con}_{F_{n} \mid F_{n-1}}\left(D_{i-1}^{(n-1)}\right) .
$$

Mas, como por hipótese $\operatorname{deg}\left(D_{i-1}^{(n-1)}\right)=q^{i-1} \cdot(q-1)$, usando o corolário 3.13 e o fato de $K$ ser algebricamente fechado em $F_{n}$, temos que:

$$
\begin{aligned}
\operatorname{deg}\left(D_{i}^{(n)}\right) & =\left[F_{n}: F_{n-1}\right] \cdot \operatorname{deg}\left(D_{i-1}^{(n-1)}\right) \\
& =q \cdot q^{i-1} \cdot(q-1) \\
& =q^{i} \cdot(q-1) .
\end{aligned}
$$

- Agora, mostraremos (por indução sobre $n$ ) que $\operatorname{deg}\left(D^{(n)}\right)=q^{\left[\frac{n}{2}\right]}$.

- Se $n=1$, temos que $D^{(1)}=P_{\infty}$, e, como $\operatorname{deg}\left(P_{\infty}\right)=1, \operatorname{temos} \operatorname{deg}\left(D^{(1)}\right)=$ $1=q^{\left[\frac{1}{2}\right]}$.

- Se $n=2$, temos que $D^{(2)}=\sum_{P \in S^{(2)}} P$; mas, como $S^{(2)}$ é formado por $q$ lugares de grau 1, temos que $\operatorname{deg}\left(D^{(2)}\right)=q=q^{\left[\frac{2}{2}\right]}$.

- Caso n ímpar:

Como $n$ é ímpar, temos que os lugares de $S^{(n)}$ são extensões biunívocas dos lugares de $S^{(n-1)}$, os quais são totalmente ramificados em $F_{n}$. Logo:

$$
\begin{aligned}
D^{(n)} & =\sum_{P \in D^{(n-1)}} \sum_{P^{\prime} \mid P} P^{\prime}=\sum_{P \in D^{(n-1)}} \sum_{P^{\prime} \mid P}\left(\frac{e\left(P^{\prime} \mid P\right)}{q} \cdot P^{\prime}\right) \\
& =\frac{1}{q} \cdot \sum_{P \in D^{(n-1)}} \sum_{P^{\prime} \mid P}\left(e\left(P^{\prime} \mid P\right) \cdot P^{\prime}\right)=\frac{1}{q} \cdot \operatorname{Con}_{F_{n} \mid F_{n-1}}\left(D^{(n-1)}\right),
\end{aligned}
$$

e, portanto, usando o corolário 3.13 e o fato de $K$ ser algebricamente fechado em $F_{n}$, temos:

$q \cdot D^{(n)}=\operatorname{Con}_{F_{n} \mid F_{n-1}}\left(D^{(n-1)}\right) \Longrightarrow q \cdot \operatorname{deg}\left(D^{(n)}\right)=\left[F_{n}: F_{n-1}\right] \cdot \operatorname{deg}\left(D^{(n-1)}\right)$.

Mas, por hipótese, temos que $\operatorname{deg}\left(D^{(n-1)}\right)=q^{\left[\frac{n-1}{2}\right]}$. Logo:

$$
q \cdot \operatorname{deg}\left(D^{(n)}\right)=q \cdot q^{\left[\frac{n-1}{2}\right]} \Longrightarrow \operatorname{deg}\left(D^{(n)}\right)=q^{\left[\frac{n-1}{2}\right]} .
$$

Contudo, como $n$ éímpar, temos $\left[\frac{n-1}{2}\right]=\left[\frac{n}{2}\right]$, e, conseqüentemente, $\operatorname{deg}\left(D^{(n)}\right)=$ $q^{\left[\frac{n}{2}\right]}$. 


\section{- Caso n par:}

Para calcular o grau de $D^{(n)}$, devemos observar que, desta vez, $S^{(n)}$ recebeu, além das extensões dos lugares de $S^{(n-1)}$, as extensões dos lugares de $S_{\left[\frac{n-4}{2}\right]}^{(n-1)}=$ $S_{\frac{n-4}{2}}^{(n-1)}$ (pois $n$ é par). Como os lugares de $S_{\frac{n-4}{2}}^{(n-1)}$ e $S^{(n-1)}$ são respectivamente não-ramificados e totalmente ramificados em $F_{n}$, temos, de forma análoga ao que fizemos no caso ímpar,

$$
D^{(n)}=\operatorname{Con}_{F_{n} \mid F_{n-1}}\left(D_{\frac{n-4}{2}}^{(n-1)}\right)+\frac{1}{q} \cdot \operatorname{Con}_{F_{n} \mid F_{n-1}}\left(D^{(n-1)}\right)
$$

o que implica que:

$$
\begin{aligned}
\operatorname{deg}\left(D^{(n)}\right) & =\operatorname{deg}\left(\operatorname{Con}_{F_{n} \mid F_{n-1}}\left(D_{\frac{n-4}{2}}^{(n-1)}\right)\right)+\frac{1}{q} \cdot \operatorname{deg}\left(\operatorname{Con}_{F_{n} \mid F_{n-1}}\left(D^{(n-1)}\right)\right) \\
& =q \cdot q^{\frac{n-4}{2}} \cdot(q-1)+\frac{1}{q} \cdot q \cdot q^{\left[\frac{n-1}{2}\right]} \\
& =q^{\frac{n-2}{2}} \cdot(q-1)+q^{\frac{n-2}{2}} \\
& =q^{\frac{n}{2}}=q^{\left[\frac{n}{2}\right]} .
\end{aligned}
$$

Lema 4.16. Se $g_{n}$ e $g_{n+1}$ são os gêneros de $F_{n}$ e $F_{n+1}$, respectivamente, então:

$$
2 g_{n+1}-2=q \cdot\left(2 g_{n}-2\right)+q^{\left[\frac{n}{2}\right]} \cdot(q+2) \cdot(q-1) .
$$

Demonstração. Pelo item (iii) da proposição 4.2 , temos que, se $P \in \mathbb{P}\left(F_{n}\right)$ e $v_{P}\left(x_{n}\right) \geq 0$, então $P$ é não-ramificado em $F_{n+1}$, ou seja, apenas os pólos de $x_{n}$ em $F_{n}$ têm possibilidade de se ramificar em $F_{n+1}$. Mas, por outro lado, vimos que os lugares de $S_{i}^{(n)}$ são nãoramificados em $F_{n+1}$. Logo, pelo item (ii) do lema 4.14, os lugares de $F_{n}$ que se ramificam em $F_{n+1}$ são exatamente os lugares de $S^{(n)}$, os quais são totalmente ramificados em $F_{n+1}$. Então, para cada lugar $P \in S^{(n)}$, temos $m=q+1$ (no contexto do item (ii) da proposição 4.2), e o expoente da diferente de $P^{\prime} \in \mathbb{P}\left(F_{n+1}\right)$ com $P^{\prime} \mid P$ será dado por $d\left(P^{\prime} \mid P\right)=$ $(q-1)(m+1)=(q-1)(q+2)$. Assim, lembrando que $\operatorname{deg}\left(P^{\prime}\right)=\operatorname{deg}(P)$, temos:

$$
\begin{aligned}
\operatorname{deg}\left(\operatorname{Diff}\left(F_{n+1} \mid F_{n}\right)\right) & =\sum_{P \in S^{(n)}} \sum_{P^{\prime} \mid P}\left(P^{\prime} \mid P\right) \cdot \operatorname{deg}\left(P^{\prime}\right) \\
& =\sum_{P \in S^{(n)}} \sum_{P^{\prime} \mid P}(q-1) \cdot(q+2) \cdot \operatorname{deg}(P) \\
& =(q-1) \cdot(q+2) \cdot \sum_{P \in S^{(n)}} \sum_{P^{\prime} \mid P} \operatorname{deg}(P) \\
& =(q-1) \cdot(q+2) \cdot \operatorname{deg}\left(D^{(n)}\right) \\
& =q^{\left[\frac{n}{2}\right]} \cdot(q+2) \cdot(q-1) .
\end{aligned}
$$


Então, pela fórmula do gênero de Hurwitz (teorema 3.36), teremos

$$
2 g_{n+1}-2=q\left(2 g_{n}-2\right)+q^{\left[\frac{n}{2}\right]} \cdot(q+2)(q-1),
$$

onde $g_{n+1}$ e $g_{n}$ são os gêneros de $F_{n+1}$ e $F_{n}$, respectivamente.

Obteremos agora o principal resultado desta seção, que pode ser considerado um dos pilares deste capítulo.

Teorema 4.17 .

$$
g_{n}=\left\{\begin{array}{l}
q^{n}+q^{n-1}-q^{\frac{n+1}{2}}-2 q^{\frac{n-1}{2}}+1, \text { se } n \text { é ímpar } \\
q^{n}+q^{n-1}-\frac{1}{2} q^{\frac{n}{2}+1}-\frac{3}{2} q^{\frac{n}{2}}-q^{\frac{n}{2}-1}+1, \text { se } n \text { é par }
\end{array} .\right.
$$

Demonstração. Procederemos por indução sobre $n$.

- Caso $n=1$ :

Temos:

$$
g_{1}=q^{1}+q^{1-1}-q^{\frac{1+1}{2}}-2 q^{\frac{1-1}{2}}+1=0 .
$$

De fato, $F_{1}=K\left(x_{1}\right)$ é um corpo de funções racionais, e, portanto, tem gênero 0 .

- Caso $n=2$ :

Temos:

$$
g_{2}=q^{2}+q^{2-1}-\frac{1}{2} q^{\frac{2}{2}+1}-\frac{3}{2} q^{\frac{2}{2}}-q^{\frac{2}{2}-1}+1=\frac{1}{2} q^{2}-\frac{1}{2} q
$$

De fato, usando o lema acima e o fato de que $g_{1}=0$, obtemos $g_{2}=\frac{1}{2} q^{2}-\frac{1}{2} q$.

Agora, suponhamos a validade da fórmula para $n \geq 2$.

- Se $n$ é par, então, por hipótese, temos que:

$$
g_{n}=q^{n}+q^{n-1}-\frac{1}{2} q^{\frac{n}{2}+1}-\frac{3}{2} q^{\frac{n}{2}}-q^{\frac{n}{2}-1}+1 .
$$

Agora, pelo lema 4.16, temos:

$$
\begin{aligned}
2 g_{n+1}-2 & =q\left(2\left(q^{n}+q^{n-1}-\frac{1}{2} q^{\frac{n}{2}+1}-\frac{3}{2} q^{\frac{n}{2}}-q^{\frac{n}{2}-1}+1\right)-2\right)+q^{\frac{n}{2}} \cdot\left(q^{2}+q-2\right) \\
& =2 q^{n+1}+2 q^{n}-q^{\frac{n}{2}+2}-3 q^{\frac{n}{2}+1}-2 q^{\frac{n}{2}}+2 q-2 q+q^{\frac{n}{2}+2}+q^{\frac{n}{2}+1}-2 q^{\frac{n}{2}} \\
& =2 q^{n+1}+2 q^{n}-2 q^{\frac{n}{2}+1}-4 q^{\frac{n}{2}}
\end{aligned}
$$

e, portanto,

$$
2 g_{n+1}=2 q^{n+1}+2 q^{n}-2 q^{\frac{n}{2}+1}-4 q^{\frac{n}{2}}+2 \Longrightarrow g_{n+1}=q^{n+1}+q^{n}-q^{\frac{n}{2}+1}-2 q^{\frac{n}{2}}+1,
$$

confirmando a validade do teorema para $n+1$ se $n$ é par. 
- Consideremos $n$ ímpar. Por hipótese, temos:

$$
g_{n}=q^{n}+q^{n-1}-q^{\frac{n+1}{2}}-2 q^{\frac{n-1}{2}}+1 .
$$

Pelo lema acima, obtemos:

$$
\begin{aligned}
2 g_{n+1}-2 & =q\left(2\left(q^{n}+q^{n-1}-q^{\frac{n+1}{2}}-2 q^{\frac{n-1}{2}}+1\right)-2\right)+q^{\frac{n-1}{2}}(q+2)(q-1) \\
& =2 q^{n+1}+2 q^{n}-2 q^{\frac{n+1}{2}+1}-4 q^{\frac{n-1}{2}+1}+2 q-2 q+q^{\frac{n-1}{2}}\left(q^{2}+q-2\right) \\
& =2 q^{n+1}+2 q^{n}-2 q^{\frac{n+3}{2}}-4 q^{\frac{n+1}{2}}+q^{\frac{n+3}{2}}+q^{\frac{n+1}{2}}-2 q^{\frac{n-1}{2}} \\
& =2 q^{n+1}+2 q^{n}-q^{\frac{n+3}{2}}-3 q^{\frac{n+1}{2}}-2 q^{\frac{n-1}{2}}
\end{aligned}
$$

e, portanto,

$$
\begin{gathered}
2 g_{n+1}=2 q^{n+1}+2 q^{n}-q^{\frac{n+3}{2}}-3 q^{\frac{n+1}{2}}-2 q^{\frac{n-1}{2}}+2 \\
\Longrightarrow g_{n+1}=q^{n+1}+q^{n}-\frac{1}{2} q^{\frac{n+1}{2}+1}-\frac{3}{2} q^{\frac{n+1}{2}}-q^{\frac{n+1}{2}-1}+1,
\end{gathered}
$$

o que prova o passo indutivo para o caso em que $n$ é ímpar.

\subsection{Os Lugares de Grau 1}

Nesta seção, apresentaremos uma estimativa para o número de lugares de grau $1 \mathrm{em}$ $F_{n} / K$. Para tanto, enunciamos o seguinte resultado:

Proposição 4.18. (A) Seja $P \in \mathbb{P}\left(F_{1}\right)$ um zero de $x_{1}-\alpha$, com $\alpha \in K \backslash\{0\}$. Então $P$ se decompõe totalmente em $F_{n} \mid F_{1}$, ou seja, existe exatamente $q^{n-1}$ extensões de $P$ em $F_{n}$ e todas de grau 1. Além disso, existem $\left(q^{2}-1\right) \cdot q^{n-1}$ lugares em $F_{n}$ originados desta forma.

(B) Os lugares $P \in S^{(2)}$ têm grau 1 e são totalmente ramificados em $F_{n} \mid F_{2}$. Logo, cada um destes lugares tem uma única extensâo em $F_{n}$ e esta tem grau 1 . O total desses lugares em $F_{n}$ é igual a q.

(C) Os $q$ lugares $P \in S_{0}^{(n)} \cup\left\{Q_{n}\right\}$ são de grau 1 .

Demonstração. (A) Se $P \in \mathbb{P}\left(F_{1}\right)$ é zero de $x_{1}-\alpha$, então $P$ também é zero de $x_{1}^{q+1}-$ $\alpha^{q+1}$. Como $\alpha \in K$, temos $\alpha^{q+1} \in \mathbb{F}_{q}$, e pelo item (iv) da proposição 4.2, a equação $\lambda^{q}+\lambda=\alpha^{q+1}$ tem $q$ raízes distintas $\lambda_{1}, \lambda_{2}, \ldots, \lambda_{q} \in K \backslash\{0\}$, e, para cada $\lambda_{i}$ $(1 \leq i \leq q)$, existe um único lugar $Q_{\lambda_{i}} \in \mathbb{P}\left(F_{2}\right)$ tal que $Q_{\lambda_{i}} \mid P, Q_{\lambda_{i}}$ é zero de $z_{2}-\lambda_{i}$, e $Q_{\lambda_{i}}$ tem grau 1. Em outras palavras, o lugar $P \in \mathbb{P}\left(F_{1}\right)$ irá se decompor em $q$ outros lugares de grau 1 em $F_{2}$.

Mostraremos agora que ocorre o mesmo com cada uma destas extensões em $F_{2}$, isto é, cada uma delas também vai se decompor em $q$ outros lugares de grau 1 em $F_{3}$. De 
fato, tomemos $Q_{\lambda} \in \mathbb{P}\left(F_{2}\right)$ zero de $z_{2}-\lambda$; como $\alpha \in K \backslash\{0\}$, teremos que $Q_{\lambda}$ é zero de $x_{2}-\frac{\lambda}{\alpha}$, pois

$$
x_{2}=\frac{z_{2}}{x_{1}} \Longrightarrow x_{2}-\frac{\lambda}{\alpha}=\frac{\left(z_{2}-\lambda\right)-\frac{\lambda}{\alpha} \cdot\left(x_{1}-\alpha\right)}{x_{1}} .
$$

Como $Q_{\lambda} \mid P$ e $v_{P}\left(x_{1}-\alpha\right)>0$, temos $v_{Q_{\lambda}}\left(x_{1}-\alpha\right)>0$, e, portanto, $v_{Q_{\lambda}}\left(x_{2}-\frac{\lambda}{\alpha}\right)=v_{Q_{\lambda}}\left(\left(z_{2}-\lambda\right)-\frac{\lambda}{\alpha} \cdot\left(x_{1}-\alpha\right)\right) \geq \min \left\{v_{Q_{\lambda}}\left(z_{2}-\lambda\right), v_{Q_{\lambda}}\left(x_{1}-\alpha\right)\right\}>0$, pois $Q_{\lambda} \mid P$ e $v_{P}\left(x_{1}-\alpha\right)>0$.

Logo $Q_{\lambda}$ é zero de $x_{2}^{q+1}-\left(\frac{\lambda}{\alpha}\right)^{q+1}$, com $\left(\frac{\lambda}{\alpha}\right)^{q+1} \in \mathbb{F}_{q}$. Utilizando o mesmo argumento usado para $P \in \mathbb{P}\left(F_{1}\right)$ zero de $x_{1}^{q+1}-\alpha^{q+1}$, concluímos que $Q_{\lambda}$ se decompõe em $q$ outros lugares de grau 1 em $F_{3}$.

Observamos que o mesmo procedimento adotado $\operatorname{com} Q_{\lambda}$ pode ser repetido com todos os outros de $F_{2}$, e mais ainda, com todas as extensões destes lugares em qualquer ponto da torre.

Portanto, acima de cada lugar de $F_{1}$, zero de $x_{1}-\alpha, \alpha \in K \backslash\{0\}$, temos exatamente $q^{n-1}$ lugares em $F_{n}$ do tipo descrito, totalizando $\left(q^{2}-1\right) \cdot q^{n-1}$.

(B) Lembrando que

$$
S_{0}^{(2)}=\left\{P \in \mathbb{P}\left(F_{2}\right): P \cap F_{1}=Q_{1} \text { e } P \neq Q_{2}\right\}
$$

e

$$
S^{(2)}=\left\{P \in \mathbb{P}\left(F_{2}\right): P \in S_{0}^{(2)} \text { ou } P \cap F_{1}=P_{\infty}\right\}
$$

observamos que $S^{(2)}$ tem $q$ lugares e todos são de grau 1. Já sabemos também, pelo item (ii) do lema 4.14, que os lugares de $S^{(2)}$ são totalmente ramificados em $F_{n}$. Logo, o índice de ramificação de cada um deles é $\left[F_{n}: F_{2}\right]=q^{n-2}$, e como $\sum e_{i} f_{i}=\left[F_{n}: F_{2}\right]$, temos que o grau relativo de cada um deles é 1 , o que garante, uma vez que $K$ é algebricamente fechado em $F_{n}$, que todas as extensões também têm grau 1 (vide figura abaixo).

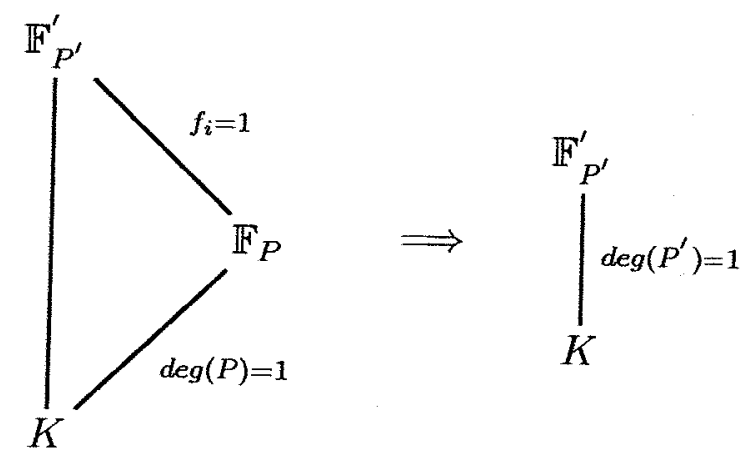
(C) Como $S_{0}^{(n)} \cup\left\{Q_{n}\right\}$ são as $q$ ramificações de $Q_{n-1}$ em $F_{n}$, segue que estas possuem
grau 1 . 
Temos uma estimativa muito boa para a quantidade de lugares de grau $1 \mathrm{em} F_{n} / K$, pois, apesar de não termos calculado efetivamente o número de lugares, veremos na seção seguinte que tal estimativa é suficiente para atingirmos a cota desejada.

Proposição 4.19. Seja $N_{n}$ o número de lugares de grau 1 em $F_{n} / K$. Para $n \geq 3$, temos

$$
N_{n} \geq\left(q^{2}-1\right) q^{n-1}+2 q
$$

Demonstração. É imediata, uma vez que temos $\left(q^{2}-1\right) q^{n-1}$ lugares do tipo $(A), q$ lugares do tipo $(B)$ e $q$ lugares do tipo $(C)$.

\subsection{Atingindo a Cota de Drinfeld-Vladut}

Agora, usando os dois pilares deste capítulo, podemos, finalmente, calcular o tão esperado limite e ver a cota de Drinfeld-Vladut ser atingida.

\section{Teorema 4.20.}

$$
A\left(q^{2}\right)=q-1
$$

Demonstração. De fato, vamos mostrar que

$$
\lim _{n \rightarrow \infty} \frac{N_{n}}{g_{n}}=q-1 \text {. }
$$

Usando o teorema 4.17 e a proposição 4.19, temos:

- Para $n$ ímpar:

$$
\begin{aligned}
\liminf _{n \rightarrow \infty} \frac{N_{n}}{g_{n}} & \geq \lim _{n \rightarrow \infty} \frac{q^{n+1}-q^{n-1}+2 q}{q^{n}+q^{n-1}-q^{\frac{n+1}{2}}-2 q^{\frac{n-1}{2}}+1} \\
& =\lim _{n \rightarrow \infty} \frac{q^{2}-1+\frac{2}{q^{n-2}}}{q+1-\frac{1}{q^{\frac{n-3}{2}}}-\frac{2}{q^{\frac{n-1}{2}}}+\frac{1}{q^{n-1}}} \\
& =\frac{q^{2}-1}{q+1}=q-1 .
\end{aligned}
$$

- Para n par:

$$
\begin{aligned}
\liminf _{n \rightarrow \infty} \frac{N_{n}}{g_{n}} & \geq \lim _{n \rightarrow \infty} \frac{q^{n+1}-q^{n-1}+2 q}{q^{n}+q^{n-1}-\frac{1}{2} q^{\frac{n}{2}+1}-\frac{3}{2} q^{\frac{n}{2}}-q^{\frac{n}{2}-1}+1} \\
& =\lim _{n \rightarrow \infty} \frac{q^{2}-1-\frac{2}{q^{n-2}}}{q+1-\frac{1}{2} \frac{1}{q^{\frac{n}{2}-2}}-\frac{3}{2} \frac{1}{q^{\frac{n-2}{2}}}-\frac{1}{q^{\frac{n}{2}}}+\frac{1}{q^{n-1}}} \\
& =\frac{q^{2}-1}{q+1}=q-1 .
\end{aligned}
$$


Portanto,

$$
\liminf _{n \rightarrow \infty} \frac{N_{n}}{g_{n}} \geq q-1 .
$$

Mas, pela cota de Drinfeld-Vladut, temos que

$$
\limsup _{n \rightarrow \infty} \frac{N_{n}}{g_{n}} \leq A\left(q^{2}\right) \leq q-1
$$

ou seja,

$$
q-1 \leq \liminf _{n \rightarrow \infty} \frac{N_{n}}{g_{n}} \leq \limsup _{n \rightarrow \infty} \frac{N_{n}}{g_{n}} \leq q-1 .
$$

Logo:

$$
\lim _{n \rightarrow \infty} \frac{N_{n}}{g_{n}}=q-1,
$$

e, conseqüentemente, $A\left(q^{2}\right)=q-1$.

\subsection{O Valor Preciso de $N_{n}$}

Acabamos de explicitar uma torre $F_{n}$ de corpos de funções sobre $\mathbb{F}_{q^{2}}$ que atinge a cota de Drinfeld-Vladut. Veremos agora que é possível calcular efetivamente a quantidade $N_{n}$ de lugares de grau 1 em $F_{n}$.

Para este cálculo, precisamos essencialmente do teorema 3.29 (de Kummer) e de alguns refinamentos nas demonstrações dos lemas 4.12 e 4.13. Nas demonstrações desta seção, evitaremos repetir justificativas de passagens feitas anteriormente.

Primeiramente, observamos que todos os lugares de grau 1 em $F_{n}$ são necessariamente extensões de lugares de grau 1 do corpo de funções racionais $F_{1}=K\left(x_{1}\right)$. Em outras palavras, os lugares de grau 1 de $F_{n}$ são extensões de lugares de $F_{1}$ que estão associados aos elementos do conjunto $K \cup\{\infty\}$.

Portanto, levando em conta a estimativa que fizemos na seção 4.3 , concluímos que só nos resta analisar os lugares $P \in S_{i}^{(n)}, 1 \leq i \leq\left[\frac{n-3}{2}\right]$, com $n \geq 5$.

Para tanto, voltaremos à demonstração da proposição 4.11 , onde temos que, se $P \in$ $S_{i}^{(n)}, 0 \leq i \leq\left[\frac{n-3}{2}\right]$, então:

$$
x_{n}^{q+1}=\wp\left(x_{n-2 i-2}^{-(q+1)}\right)+\mathcal{O}(1) \text { em } P .
$$

Observaremos que, se escrevermos

$$
x_{n}^{q+1}=\wp\left(x_{n-2 i-2}^{-(q+1)}\right)+\lambda+v
$$

com $\lambda \in K$ e $v_{P}(z)>0$, então o comportamento de $P$ na extensão $F_{n+1} \mid F_{n}$ dependerá de $\lambda$ no seguinte sentido:

Proposição 4.21. Um lugar de grau $1 P \in S_{i}^{(\bar{n})}$ decompõe-se em $q$ outros lugares de grau 1 de $F_{n+1}$ se, e somente se, $\lambda \in \mathbb{F}_{q}$. 
Demonstração. Fixado $i$, tomemos $u:=x_{n-2 i-2}^{-(q+1)}$ em $F_{n}$. Temos então:

$$
\begin{aligned}
x_{n}^{q+1}=u^{q}+u+\lambda+v & \Longrightarrow z_{n+1}^{q}+z_{n+1}=u^{q}+u+\lambda+v \\
& \Longrightarrow\left(z_{n+1}-u\right)^{q}+\left(z_{n+1}-u\right)=\lambda+v .
\end{aligned}
$$

Ao fazermos $\bar{z}_{n+1}:=z_{n+1}-u$, temos que $F_{n+1}=F_{n}\left(\bar{z}_{n+1}\right)$ e que $\varphi(T)=T^{q}+T-\lambda-v$ é o polinômio minimal de $\bar{z}_{n+1}$ sobre $F_{n}$.

Assim, usando os resultados, bem como a notação, do teorema 3.29 (de Kummer), temos que $\bar{z}_{n+1}$ é inteiro sobre $O_{P}$ e que $\bar{\varphi}(T)=T^{q}+T-\bar{\lambda}$. Então, pelo teorema de Kummer, o lugar $P$ decompõe-se em $q$ outros lugares de grau 1 se, e somente se, o polinômio $T^{q}+T-\lambda$ fatora-se linearmente em $O_{P}[T]$, e é fácil ver que isto ocorre se, e somente se, $\lambda \in \mathbb{F}_{q}$. De fato:

○ Se $\lambda \in \mathbb{F}_{q}$, e $\omega \in \overline{\mathbb{F}}_{q}$ é tal que $\omega^{q}+\omega=\lambda$, então:

$$
\left(\omega^{q}+\omega\right)^{q}=\lambda^{q} \Longleftrightarrow \omega^{q^{2}}+\omega^{q}=\lambda \Longleftrightarrow \omega^{q^{2}}=\omega \Longleftrightarrow \omega \in K .
$$

Portanto, o polinômio $T^{q}+T-\lambda$ tem todas as raízes em $K$.

- Se $\lambda \notin \mathbb{F}_{q}$, isto é, se $\lambda \in K \backslash \mathbb{F}_{q}$, então, obviamente, $T^{q}+T-\lambda$ não tem raízes em $K$ e, conseqüentemente, não tem raízes em $O_{P}$, pois $K$ é algebricamente fechado em $\mathbb{F}_{n}$. Logo, nenhuma extensão de $P$ tem grau 1.

As seguintes afirmações serão utilizados no decorrer de nossos cálculos:

AFIRMAÇÃo 1: Na notação do lema 4.12 (II), temos que

$$
x_{k}^{-(q+1)}=\wp\left(x_{k-1}^{-(q+1)}\right)+s,
$$

onde $v_{P}(s) \geq 0$. Afirmamos que, na verdade, temos $v_{P}(s)>0$.

Demonstração. Basta refinar um pouco a demonstração do lema 4.12 (II), a qual nos remete às demonstrações das propriedades $P_{6}$ e $P_{7}$ (seção 3.8).

Inicialmente, temos que:

$$
x_{k}=x_{k-1}^{q} \cdot\left(1-y^{q-1}+y^{q^{2}-1} \cdot r\right),
$$

onde $v_{P}(r) \geq 0$. Mas, como $v_{P}(y)>0$, podemos escrever

$$
y^{q^{2}-1} \cdot r=y^{q} \cdot y^{q^{2}-q-1} \cdot r=y^{q} \cdot t,
$$

$\operatorname{com} v_{P}(t)>0$. Agora, pela demonstração de $P_{6}$, temos

$$
\left(1-y^{q-1}+y^{q} \cdot t\right)^{-1}=1+y^{q-1}+y^{q} \cdot t^{\prime}
$$

onde

$$
t^{\prime}=\frac{y^{q-2}-t y^{q-1}-t}{1-y^{q-1}+y^{q} \cdot t}
$$


e, na demonstração de $P_{7}$, vemos que

$$
s=t^{\prime}+y^{q^{2}-2 q}+y^{q^{2}-q} \cdot t^{\prime q}+\left(y^{q^{2}-2 q}+y^{q^{2}-q} \cdot t^{\prime q}\right)\left(y^{q-1}+y^{q} \cdot t^{\prime}\right) .
$$

Como $v_{P}(y)>0$ e $v_{P}(t)>0$, segue imediatamente que $v_{P}(s)>0$ para $q>2$.

Agora, caso tenhamos $q=2$, teremos

$$
t^{\prime}=\frac{1+t y+t}{1+y+y^{2} t}
$$

e

$$
s=t^{\prime}+1+y^{2} t^{2}+\left(1+y^{2} t^{2}\right)\left(y+y^{2} t^{\prime}\right) .
$$

Mas, apesar de termos $v_{P}\left(t^{\prime}\right)=0$, é fácil ver que $v_{P}\left(t^{\prime}+1\right)>0$, pois

$$
t^{\prime}+1=\frac{y^{2} t+t y+y+t}{1+y+y^{2} t}
$$

e, conseqüentemente, temos $v_{P}(s)>0$.

AFIRMAÇÃO 2: Usando a notação do lema 4.12 (I), temos, para $n \geq 4$, que

$$
x_{n-2}=x_{n-3}^{q} \cdot\left(1+x_{n-3}^{q^{2}-1} \cdot t\right),
$$

onde $v_{P}(t) \geq 0$, e $P \in \mathbb{P}\left(F_{n}\right)$ é tal que $P \mid Q_{n-2}$. Se $q=2$, afirmamos que, necessariamente, temos $t+1 \in P$.

Demonstração. Pela demonstração do lema 4.12, temos que

$$
t=\left(\frac{z_{n-2}}{x_{n-3}^{q+1}}\right)^{q}
$$

Sendo $q=2$, temos :

$$
\begin{aligned}
t=\left(\frac{z_{n-2}}{x_{n-3}^{3}}\right)^{2} & \Longrightarrow t+1=\left(\frac{z_{n-2}}{x_{n-3}^{3}}+1\right)^{2} \\
& \Longrightarrow t+1=\left(\frac{z_{n-2}+x_{n-3}^{3}}{x_{n-3}^{3}}\right)^{2}
\end{aligned}
$$

Por outro lado, temos

$$
z_{n-2}^{2}+z_{n-2}=x_{n-3}^{3} \Longrightarrow z_{n-2}+x_{n-3}^{3}=z_{n-2}^{2} .
$$

Logo,

$$
t+1=\left(\frac{z_{n-2}^{2}}{x_{n-3}^{3}}\right)^{2}=z_{n-2}^{2} \cdot t,
$$

e, como $v_{P}\left(z_{n-2}\right)>0$, temos $v_{P}(t+1)>0 \Longrightarrow t+1 \in P$. 
Proposição 4.22. Se q é ímpar e $n \geq 3$, temos $N_{n}=\left(q^{2}-1\right) \cdot q^{n-1}+2 q$.

Demonstração. Tomando $P=Q_{\alpha} \in S_{0}^{(n)}$ (ou seja, $P$ é zero comum de $x_{1}, z_{2}, \ldots, z_{n-1}$ e $z_{n}-\alpha$, onde $\alpha^{q}+\alpha=0$ e $\left.\alpha \in K \backslash\{0\}\right)$, temos, pela demonstração do lema 4.13 , caso $i=0(\mathrm{em} *)$, que

$$
x_{n}^{q+1}=\frac{\alpha^{q+1}}{x_{n-1}^{q+1}}+\alpha^{q}+z,
$$

onde $v_{P}(z)>0$, e assim, pela demonstração da proposição 4.11 (a qual nos remete ao lema 4.12(II)), temos

$$
x_{n}^{q+1}=\wp\left(\gamma \cdot x_{n-2}^{-(q+1)}\right)+\alpha^{q}+z^{\prime},
$$

com $\gamma \in \mathbb{F}_{q} \backslash\{0\}$ e $v_{P}\left(z^{\prime}\right)>0$ (tal desigualdade estrita se justifica pela afirmação 1 ). Usando a notação da proposição 4.21, concluímos assim que $\lambda=\alpha^{q}$.

Mas, como $\alpha^{q}+\alpha=0$ e $\alpha \in K \backslash\{0\}$, temos

$$
\alpha^{q} \in \mathbb{F}_{q} \Longleftrightarrow \alpha^{q^{2}}=\alpha^{q} \Longleftrightarrow \alpha=\alpha^{q} \Longleftrightarrow 2 \alpha=0 \Longleftrightarrow K \text { tem característica } 2 \text {. }
$$

Portanto, se $q$ é ímpar, pela proposição 4.21, nenhuma extensão dos lugares de $S_{0}^{(n)}$ tem grau 1, e, conseqüentemente, nenhum lugar de $F_{n}$, que é extensão de lugares de $S_{0}^{(m)}$, com $3 \leq m \leq n-1$, possui grau 1. Assim, aquela desigualdade que ocorre na estimativa da seção 4.3 , é precisamente uma igualdade, ou seja, $N_{n}=\left(q^{2}-1\right) q^{n-1}+2 q$.

Veremos agora o caso $q \equiv 0(\bmod 2)$. Neste caso, se $P \in S_{0}^{(n-1)}$, com $n \geq 4$, pelo que foi demonstrado acima, temos por (1) que

$$
x_{n-1}^{q+1}=\wp\left(\gamma \cdot x_{n-3}^{-(q+1)}\right)+\alpha^{q}+z
$$

onde $v_{P}(z)>0$, ou seja, $P$ decompõe-se em $q$ outros lugares de grau 1 , que irão compor o $S_{1}^{(n)}$. Nos resta, agora, analisar o que ocorre, em termos de grau, com as extensões dos lugares de $S_{1}^{(n)}$.

Proposição 4.23. Suponha car $(K)=2$. A constante $\lambda^{\prime} \in K$, que ocorre nas extensões dos lugares de $S_{1}^{(n)}$ (conforme a proposição 4.21), satisfaz:

$$
\lambda^{\prime}=\left\{\begin{array}{l}
\beta, \text { se } q>2 \\
\beta+1, \text { se } q=2
\end{array}\right.
$$

onde $\beta^{q}+\beta=\alpha^{q}$. Logo, $\lambda^{\prime} \notin \mathbb{F}_{q}$, e nenhum destes lugares se decompõe em $q$ outros de grau 1.

Demonstração. Como $\alpha^{q}+\alpha=0$ e $\alpha \in K \backslash\{0\}$, e assumindo (*), temos:

$$
\lambda^{\prime} \in \mathbb{F}^{q} \Longleftrightarrow\left(\lambda^{\prime}\right)^{q}=\lambda^{\prime} \Longleftrightarrow \beta^{q}=\beta \Longleftrightarrow \alpha=0 \text {. }
$$


Logo, $\lambda^{\prime} \notin \mathbb{F}_{q}$, ou seja, se $\operatorname{car}(K)=2$, então (pela proposição 4.21 ) os lugares de $S_{1}^{(n)}$ não terão extensões de grau 1 em $F_{n+1}$.

Agora, provaremos (*). Já vimos anteriormente que, se $P \in S_{0}^{(n-1)}, n \geq 4$, temos:

$$
x_{n-1}^{q+1}=\wp\left(\gamma \cdot x_{n-3}^{(n-1)}\right)+\alpha^{q}+z,
$$

com $v_{P}(z)>0$. Como $\wp\left(z_{n}\right)=x_{n-1}^{q+1}$, temos:

$$
\wp\left(z_{n}\right)=\wp\left(\gamma \cdot x_{n-3}^{-(q+1)}\right)+\alpha^{q}+z \Longrightarrow \wp\left(z_{n}-\gamma \cdot x_{n-3}^{-(q+1)}\right)=\alpha^{q}+z,
$$

com $v_{P}(z)>0$. Veremos agora o que ocorre com os lugares $P^{\prime}$ que formam o $S_{1}^{(n)}$, isto é, são extensões dos lugares $P \in S_{0}^{(n-1)}$.

Observação: Doravante, por conveniência, denotaremos qualquer elemento de $F_{n}$ que está em $P^{\prime}$ por $z^{\prime}$.

Tomemos $P^{\prime} \in S_{1}^{(n)}$ tal que $P^{\prime} \mid P$. O teorema $3.29(\mathrm{a})\left(\varphi_{i}(y) \in P_{i}\right)$ nos garante que existe $\beta \in K$, com $\beta^{q}+\beta=\alpha^{q}$, tal que:

$$
z_{n}-\gamma \cdot x_{n-3}^{-(q+1)}-\beta \in P^{\prime}
$$

isto é,

$$
z_{n}=\gamma \cdot x_{n-3}^{-(q+1)}+\beta+z^{\prime} \text {. }
$$

Observe que, como $P^{\prime} \in S_{1}^{(n)}$, estamos no caso $i=1$ da demonstração do lema 4.13, mais precisamente em (2) da demonstração de tal lema.

Lembrando que $x_{n}^{q+1}=A^{q+1} \cdot B^{-1}$, onde $A:=z_{n} \cdot x_{n-2}$ e $B:=x_{n-1}^{q+1} \cdot x_{n-2}^{q+1}$, faremos um refinamento (modificação) de $A^{q+1}$ e $B^{-1}$ em relação ao que foi apresentado no lema 4.13 .

- Refinando $A^{q+1}$ :

Pelo item (I) do lema 4.12, temos que

$$
x_{n-2}=x_{n-3}^{q} \cdot\left(1+x_{n-3}^{q^{2}-1} \cdot t\right),
$$

onde $v_{P^{\prime}}(t) \geq 0$. Então:

$$
\begin{aligned}
A & =z_{n} \cdot x_{n-2} \\
& =\left(\gamma \cdot x_{n-3}^{-(q+1)}+\beta+z^{\prime}\right) \cdot x_{n-3}^{q} \cdot\left(1+x_{n-3}^{q^{2}-1} \cdot t\right) \\
& =\left(\gamma \cdot x_{n-3}^{-1}+\left(\beta+z^{\prime}\right) \cdot x_{n-3}^{q}\right) \cdot\left(1+x_{n-3}^{q^{2}-1} \cdot t\right) \\
& =\gamma \cdot x_{n-3}^{-1}+\left(\beta+z^{\prime}\right) \cdot x_{n-3}^{q}+\gamma \cdot t \cdot x_{n-3}^{q^{2}-2}+t \cdot\left(\beta+z^{\prime}\right) \cdot x_{n-3}^{q^{2}+q-1} \\
& =\gamma \cdot x_{n-3}^{-1}+x_{n-3}^{q} \cdot\left(\beta+z^{\prime}+\gamma \cdot t \cdot x_{n-3}^{q^{2}-q-2}+t \cdot\left(\beta+z^{\prime}\right) \cdot x_{n-3}^{q^{2}-1}\right) .
\end{aligned}
$$

Portanto:

$$
A=\gamma \cdot x_{n-3}^{-1}+x_{n-3}^{q} \cdot\left(\lambda^{\prime}+z^{\prime}\right),
$$


onde

(*) $\lambda^{\prime}=\left\{\begin{array}{l}\beta \text { se } q>2 \\ \beta+1 \text { se } q=2 \text { (este caso segue da afirmação 2) }\end{array}\right.$

Logo:

$$
\begin{aligned}
A^{q+1} & =\left(\gamma^{q} \cdot x_{n-3}^{-q}+x_{n-3}^{q^{2}} \cdot\left(\lambda^{\prime}+z^{\prime}\right)^{q}\right) \cdot\left(\gamma \cdot x_{n-3}^{-1}+x_{n-3}^{q} \cdot\left(\lambda^{\prime}+z^{\prime}\right)\right) \\
& =\gamma^{q+1} \cdot x_{n-3}^{-(q+1)}+\gamma^{q} \cdot\left(\lambda^{\prime}+z^{\prime}\right)+\underbrace{\left(\lambda^{\prime}+z^{\prime}\right)^{q} \cdot x_{n-3}^{q^{2}} \cdot\left(\gamma \cdot x_{n-3}^{-1}+x_{n-3}^{q} \cdot\left(\lambda^{\prime}+z^{\prime}\right)\right)}_{\in P^{\prime}} \\
& =\gamma^{2} \cdot x_{n-3}^{-(q+1)}+\gamma^{q} \cdot \lambda^{\prime}+z^{\prime} .
\end{aligned}
$$

- Refinando $B^{-1}$ :

Sabemos que $B^{-1}=\gamma^{-1}+x_{n-2}^{q+1} \cdot s$, onde $v_{P^{\prime}}(s) \geq 0$. Mas, na demonstração do lema $4.13 \mathrm{em}(4)$, temos que $x_{n-2}=t \cdot x_{n-3}$, onde $v_{P^{\prime}}(t)>0$. Logo, $B^{-1}=\gamma^{-1}+x_{n-3}^{q+1} \cdot z^{\prime}$. Finalmente, temos:

$$
\begin{aligned}
x_{n+1}^{q+1} & =A^{q+1} \cdot B^{-1} \\
& =\left(\gamma^{2} \cdot x_{n-3}^{-(q+1)}+\gamma^{q} \cdot \lambda^{\prime}+z^{\prime}\right)\left(\gamma^{-1}+x_{n-3}^{q+1} \cdot z^{\prime}\right) \\
& =\gamma \cdot x_{n-3}^{-(q+1)}+\gamma^{q-1} \cdot \lambda^{\prime}+\underbrace{z^{\prime} \cdot \gamma^{-1}+\gamma^{2} \cdot z^{\prime}+\left(\gamma^{q} \cdot \lambda^{\prime}+z^{\prime}\right) \cdot z^{\prime} \cdot x_{n-3}^{q+1}}_{\in P^{\prime}} \\
& =\gamma \cdot x_{n-3}^{-(q+1)}+\lambda^{\prime}+z^{\prime} .
\end{aligned}
$$

Agora, pela afirmação 1, temos:

$$
x_{n-3}^{-(q+1)}=\wp\left(x_{n-4}^{-(q+1)}\right)+z^{\prime},
$$

e, portanto,

$$
x_{n+1}^{q+1}=\wp\left(\gamma \cdot x_{n-4}^{-(q+1)}\right)+\lambda^{\prime}+z^{\prime}
$$

onde

$$
\lambda^{\prime}=\left\{\begin{array}{l}
\beta \text { se } q>2 \\
\beta+1 \text { se } q=2
\end{array}\right.
$$

$\operatorname{com} \beta^{q}+\beta=\alpha^{q}$

Corolário 4.24. O número $N_{n}$ de lugares de grau 1 em $F_{n}$ é dado por $N_{1}=q^{2}+1 e$ $N_{2}=q^{3}+1$ (que é,naturalmente, o número de pontos $K$-racionais da curva hermitiana; ver (4]). Além disso, conforme a paridade de $q$, temos ainda que:

(I) $q$ ímpar:

$$
N_{n}=\left(q^{2}-1\right) \cdot q^{n-1}+2 q \text { para } n \geq 3 \text { (que é a cota obtida anteriormente) ; }
$$


(II) $q$ par:

(i) $N_{3}=\left(q^{2}-1\right) \cdot q^{2}+2 q$;

(ii) $N_{4}=\left(q^{2}-1\right) \cdot q^{3}+q^{2}+q$;

(iii) $N_{n}=\left(q^{2}-1\right) \cdot q^{n-1}+2 q^{2}$ para $n \geq 5$.

\section{Demonstração.}

(I) $q$ ímpar:

Segue da proposição 4.22.

(II) $q$ par:

(i) $N_{3}=\left(q^{2}-1\right) \cdot q^{2}+2 q$, pois, quando $n=3$, nada muda em relação ao caso $q$ ímpar;

(ii) $N_{4}=\left(q^{2}-1\right) \cdot q^{3}+q^{2}+q$, pois, comparado ao caso $q$ ímpar, $N_{4}$ recebeu mais $q \cdot(q-1)$ lugares de grau 1 , que são extensões dos lugares de $S_{0}^{(3)}$;

(iii) para $n \geq 5$, teremos que $N_{n}=\left(q^{2}-1\right) \cdot q^{n-1}+2 q^{2}$, pois, comparado ao caso $q \equiv 1(\bmod 2)$, teremos um aumento de lugares que são extensões dos lugares de $S_{0}^{(n-1)}$ e $S_{0}^{(3)}$, isto é, teremos um aumento de $q \cdot(q-1)+q \cdot(q-1)=2 q^{2}-2 q$ lugares de grau 1 em relação ao caso $q$ ímpar. 


\section{Apêndice: Códigos Lineares}

Definição 1. Um código linear $C$ sobre um alfabeto $\mathbb{F}_{q}$ (corpo finito de $q$ elementos) é um subespaço vetorial de $\mathbb{F}_{q}^{n}$. Se $k$ é a dimensão de $C$ sobre $\mathbb{F}_{q}$, dizemos que $C$ é um código $[n ; k]$.

Definição 2. (i) Se $a=\left(a_{1}, \ldots, a_{n}\right)$ e $b=\left(b_{1}, \ldots, b_{n}\right)$ são elementos de $\mathbb{F}_{q}^{n}$, definimos a função

$$
d(a, b):=\#\left\{i: a_{i} \neq b_{i}\right\}
$$

denominada métrica de Hamming em $\mathbb{F}_{\boldsymbol{q}}^{\boldsymbol{n}}$.

(ii) O peso de um elemento $a \in \mathbb{F}_{q}^{n}$ é definido por

$$
\omega(a):=\#\left\{i: a_{i} \neq 0\right\}
$$

(iii) A distância mínima de um código $C$ é dada por:

$$
d(C):=\min \{\omega(a): 0 \neq a \in C\} .
$$

Um código $[n ; k]$ com distância mínima d é dito um código $[n ; k ; d]$.

A distância mínima $d$ de um código $C$ está diretamente ligada à performance do código no que diz respeito à detecção e correção de erros. Neste sentido, temos o seguinte resultado:

Teorema 3. (ver [7]) Seja $C$ um código linear de distância mínima d. Então $C$ pode corrigir até $\varepsilon=\left[\frac{d-1}{2}\right]$ erros, e detectar até $d-1$ erros.

Em virtude do teorema acima, vemos que um código terá maior capacidade de correção de erros quanto maior for a sua distância mínima. Portanto, é fundamental para a teoria de códigos poder calcular $d$, ou pelo menos determinar uma cota mínima para ele.

Se $C$ é um código $[n, k, d]$, dizemos que $n, k$ e $d$ são os parâmetros fundamentais de $C$.

Para a teoria, os códigos interessantes são aqueles que apresentam valores de $k$ e $d$ "grandes" em relação a $n$. No entanto, dados três inteiros positivos $n, k$ e $d$, nem sempre existe um código $C$ que tenha tais parâmetros, pois há uma interdependência complexa entre estes três números, e um dos problemas fundamentais da teoria de códigos é o de estudá-la. 
Um exemplo simples desta interdependência é conhecido como "cota de Singleton", que estabelece, em um código $[n ; k ; d]$ qualquer, a seguinte desigualdade:

$$
k+d \leq n+1 \text {. }
$$

A demonstração segue de conceitos básicos de Álgebra Linear, e pode ser vista em [11] (página 41).

Em 1975, Goppa observou que seria possível utilizar corpos de funções algébricas sobre $\mathbb{F}_{q}$ para a construção de códigos. As propriedades destes códigos estão estreitamente relacionadas com as propriedades do corpo de funções correspondente. Nesta construção, o teorema de Riemann-Roch fornece estimativas (muito boas em alguns casos) para os parâmetros fundamentais.

Como ilustração, apresentamos a seguir a associação de um código feita a partir de um corpo de funções algébricas. Tal código é chamado de código geométrico de Goppa.

Inicialmente, fixaremos algumas notações a serem usadas nesta construção:

- $F / \mathbb{F}_{q}$ é um corpo de funções algébricas de gênero $g$;

- $P_{1}, \ldots, P_{n}$ são lugares dois a dois distintos de $F / \mathbb{F}_{q}$ de grau 1 ;

- $D=P_{1}+P_{2}+\ldots+P_{n}$

- $G$ é um divisor de $F / \mathbb{F}_{q}$ tal que $\operatorname{supp}(G) \cap \operatorname{supp}(D)=\varnothing$.

Definição 4. $O$ código geométrico de $G o p p a C_{\mathcal{L}}(D, G)$ associado aos divisores $D$ e $G$ é definido por:

$$
C_{\mathcal{L}}(D, G):=\left\{\left(x\left(P_{1}\right), \ldots, x\left(P_{n}\right)\right): x \in \mathcal{L}(G)\right\} \subseteq \mathbb{F}_{q}^{n}
$$

Vemos que esta definição, de fato, faz sentido, pois, como $x \in \mathcal{L}(G)$ e $\operatorname{supp}(G) \cap$ $\operatorname{supp}(D)=\varnothing$, temos que $v_{P_{i}}(x) \geq 0$, e, como $\operatorname{deg}\left(P_{i}\right)=1$, temos que $x\left(P_{i}\right) \in \mathbb{F}_{q}$.

Considerando a função $f: \mathcal{L}(G) \longrightarrow \mathbb{F}_{q}^{n}$, dada por

$$
f(x):=\left(x\left(P_{1}\right), \ldots, x\left(P_{n}\right)\right) \in \mathbb{F}_{q}^{n}
$$

temos que $f$ é $\mathbb{F}_{q}$-linear, e que $C_{\mathcal{L}}(D, G)$ é a imagem de $\mathcal{L}(G)$ por $f$, portanto, um subespaço vetorial de $\mathbb{F}_{q}^{n}$. Os resultados a seguir podem ser vistos em [11] (página 100).

Teorema 5. $C_{\mathcal{L}}(D, G)$ é um código $[n, k, d]$ com parâmetros $k=\operatorname{dim}(G)-\operatorname{dim}(G-D)$ e $d \geq n-\operatorname{deg}(G)$.

Corolário 6. Se $\operatorname{deg}(G)<n$, então a função $f: \mathcal{L}(G) \longrightarrow C_{\mathcal{L}}(D, G)$ é injetora, e temos que $C_{\mathcal{L}}(D, G)$ é um código $[n ; k ; d]$, com $d \geq n-\operatorname{deg}(G)$ e $k=\operatorname{dim}(G) \geq \operatorname{deg}(G)+1-g$. Portanto, $n+1-g \leq k+d \leq n+1$. 
Sendo $C$ um código $[n ; k ; d]$, definimos a taxa de correção de erros e a taxa de informação de $C$ pelos números

$$
\delta(C):=\frac{d}{n} \quad \text { e } \quad R(C):=\frac{k}{n},
$$

respectivamente.

Definição 7. Diremos que uma familia de códigos sobre $\mathbb{F}_{q}$ é assintoticamente boa se contiver uma seqüência de códigos $C_{i}$ com parâmetros $\left[n_{i} ; k_{i} ; d_{i}\right]$ para $i \in \mathbb{N}$, com $\lim _{i \rightarrow \infty} n_{i}=\infty$, e tal que os limites

$$
\lim _{i \rightarrow \infty} \delta\left(C_{i}\right) \quad e \quad \lim _{i \rightarrow \infty} R\left(C_{i}\right)
$$

existem e são positivos.

Definiremos ainda os seguintes conjuntos:

$$
V_{q}:=\left\{(\delta(C) ; R(C)) \in[0,1]^{2}: C \text { é um código sobre } \mathbb{F}_{q}\right\},
$$

e $U_{q} \subseteq[0,1]^{2}$ como sendo o conjunto dos pontos limites de $V_{q}$, ou seja,

$$
U_{q}:=\left\{(x, y) \in[0,1]^{2}: \begin{array}{ll}
\text { existe uma seqüência de códigos } C_{i} \text { sobre } \\
\mathbb{F}_{q} \text { tal que } \lim _{i \rightarrow \infty}\left(\delta\left(C_{i}\right), R\left(C_{i}\right)\right)=(x, y)
\end{array}\right\} .
$$

Uma proposição devida a Manin [11] (página 205), afirma que existe uma função contínua $\alpha_{q}:[0,1] \longrightarrow[0,1]$ tal que

$$
U_{q}=\left\{(\delta, R): 0 \leq \delta \leq 1 \text { e } 0 \leq R \leq \alpha_{q}(\delta)\right\} \text {, }
$$

e ainda $\alpha_{q}(0)=1, \alpha_{q}(\delta)=0$ para $1-\frac{1}{q} \leq \delta \leq 1$, e $\alpha_{q}$ é decrescente no intervalo $0 \leq \delta \leq 1-\frac{1}{q}$ (vide figura $I$ a seguir).

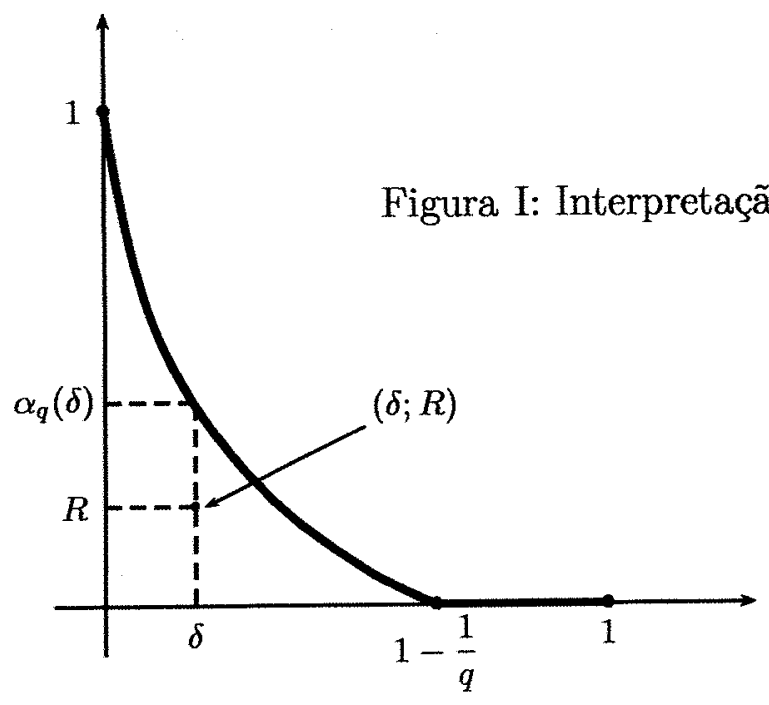

Ao escolhermos $\delta \in] 0 ; 1-\frac{1}{q}[$, e em seguida $\left.R \in] 0 ; \alpha_{q}(\delta)\right]$, o resultado de Manin afirma 
que $(\delta, R)$ é ponto limite de uma seqüência de códigos assintoticamente boa sobre $\mathbb{F}_{q}$.

$\mathrm{O}$ fato de conhecermos muito pouco sobre a função $\alpha_{q}$ nos traz o seguinte problema: imaginemos que, dado $\delta \in] 0,1-\frac{1}{q}\left[\right.$, queiramos tomar $R \in[0,1]$ tal que $0<R \leq \alpha_{q}(\delta)$; isto, a priori não é fácil, caso não tenhamos pelo menos uma cota inferior razoável para $\alpha_{q}(\delta)$. Daí, surge a idéia de nos valermos de funções simples para estimar $\alpha_{q}(\delta)$. Nesta direção, se apresenta a cota de Gilbert-Varshamov, que afirma que, para $\delta \in\left[0,1-\frac{1}{q}\right]$,
temos:

$$
\alpha_{q}(\delta) \geq 1-H_{q}(\delta)
$$

onde

$$
H_{q}(x)=\left\{\begin{array}{l}
0, \text { se } x=0 \\
\log \frac{(q-1)}{x^{x} \cdot(1-x)^{1-x}}, \text { se } 0<x<1-\frac{1}{q} .
\end{array} .\right.
$$

Durante três décadas, pensou-se que $\alpha_{q}(\delta)=1-H_{q}(\delta)$.

Com a construção dos códigos geométricos de Goppa (o código $C_{\mathcal{L}}(D, G)$ apresentado como ilustração) é possível mostrar (vide [11], página 207) que, se $A(q)>1$, então $\alpha_{q}(\delta) \geq\left(1-A(q)^{-1}\right)-\delta$ no intervalo $0 \leq \delta \leq 1-A(q)^{-1}$.

Por outro lado, sabemos que, se $q$ é um quadrado, então $A(q)=\sqrt{q}-1$, e conseqüentemente

$$
\alpha_{q}(\delta) \geq\left(1-\frac{1}{\sqrt{q}-1}\right)-\delta \text { para } 0 \leq \delta \leq 1-\frac{1}{\sqrt{q}-1} .
$$

Esta cota é chamada de cota de Tsfasman-Vladut-Zink, e o mais interessante desta cota é que, para $q \geq 49$, ela é melhor que a cota de Gilbert-Varshamov, num certo intervalo (ver figura II abaixo).

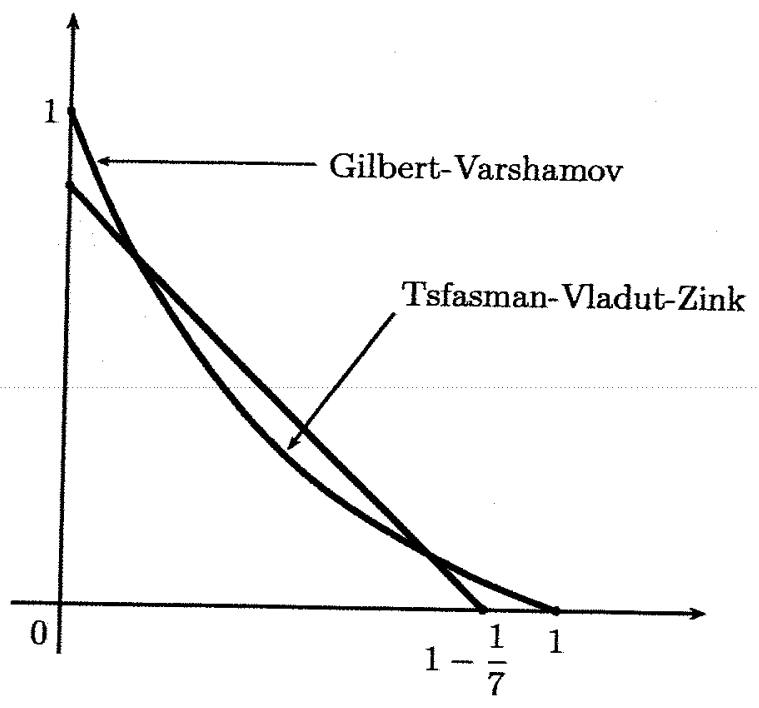

Figura II: Comparação entre as cotas de Gilbert-Varshamov e de Tsfasman-Vladut-Zink para $q=64$. 


\section{Referências Bibliográficas}

[1] Bombiere, E., Hilbert's $8^{\text {th }}$ problem an analogue in mathematical developments arising from Hilbert's problems, F.Brouder, Ed. Proc. Sym. Pure Math., vol.28, AMS, 1976, 269-274.

[2] Drinfeld, V. G., Vladut, S. G., Sobre o número de pontos de uma curva algébrica (em russo), Func. Anal. and Appl. 17 ,(1983), 68-69.

[3] Garcia, A., Stichtenoth, H., A tower of Artin-Schreier extensions of functions fields attaining the Drinfeld- Vladut bound, Invent. Math. 121,(1995), 211-222 .

[4] Garcia, A., Pontos Racionais em Curvas Sobre Corpos Finitos, $20^{\circ}$ Colóquio Brasileiro de Matemática, 1995.

[5] Goppa,V. D., Algebraico-Geometric codes, Math. U.S.S.R.,Izvestya 21,(1983),75-91.

[6] Goppa, V. D., Codes and algebraic curves, Math. U.S.S.R.,Izvestya 21,(1983),75-91.

[7] Hefez, A. , Vilella, M. L., Códigos corretores de erros, IMPA,2002.

[8] Ihara, Y., Some remarks on the number of rational points of algebraic curves over finite fields, J. Fac. Sci. Tokyo, 28,(1981), 721-724.

[9] Schoof, R., Algebraic curves over $F_{2}$ with many rational points, J. of Number Th., 41, (1992),6-14 .

[10] Serre, J.-P., Sur le nombre des points rationels d'une courbe algébrique sur un corps fini. C., R. Acad. Sci. Paris, 296,(1983), 397-402.

[11] Stichtenoth, H., Algebraic Function Ffields and Codes, Springer Universitext,1993.

[12] Tsfasman, M. A.,Vladut, S. G., Zink, T., Modular Curves, Shimura Curves and Goppa Codes, Better than Varshamov-Gilbert Bound, Math. Nachr., 109,(1982), 2128.

[13] Weil, A., Courbes Algébriques et Variétés Abeliennes, Hermann, Paris,1971.

[14] Xing, C. P., Multiple Kummer extensions of prime divisors of degree one in function fields, J. of Pure and Appl. Algebra, 84, (1993), 85-93. 